\title{
Rule-based Model Transformation from UML+MARTE to Core Scenario Models
}

\author{
by \\ Omar Mahmoud
}

A Thesis submitted to the Faculty of Graduate Studies and Research

in partial fulfillment of the requirements for the degree of

\section{Masters of Applied Science in Electrical Engineering}

Ottawa-Carleton Institute for Electrical and Computer Engineering Department of Systems and Computer Engineering

Carleton University

Ottawa, ON, Canada

September 2009

CCopyright 2009, Omar Mahmoud 
Library and Archives

Canada

Published Heritage

Branch

395 Wellington Street Ottawa ON K1A 0N4 Canada
Bibliotheqque et

Archives Canada

Direction du

Patrimoine de l'édition

395 , rue Wellington

Ottawa ON K1A ON4

Canada
Your file Votre reférence

ISBN: 978-0-494-60265-2

Our file Notre reférence

ISBN: 978-0-494-60265-2
NOTICE:

The author has granted a nonexclusive license allowing Library and Archives Canada to reproduce, publish, archive, preserve, conserve, communicate to the public by telecommunication or on the Internet, loan, distribute and sell theses worldwide, for commercial or noncommercial purposes, in microform, paper, electronic and/or any other formats.

The author retains copyright ownership and moral rights in this thesis. Neither the thesis nor substantial extracts from it may be printed or otherwise reproduced without the author's permission.
AVIS:

L'auteur a accordé une licence non exclusive permettant à la Bibliothèque et Archives Canada de reproduire, publier, archiver, sauvegarder, conserver, transmettre au public par télécommunication ou par l'Internet, prêter, distribuer et vendre des thèses partout dans le monde, à des fins commerciales ou autres, sur support microforme, papier, électronique et/ou autres formats.

L'auteur conserve la propriété du droit d'auteur et des droits moraux qui protège cette thèse. $\mathrm{Ni}$ la thèse ni des extraits substantiels de celle-ci ne doivent être imprimés ou autrement reproduits sans son autorisation.
In compliance with the Canadian Privacy Act some supporting forms may have been removed from this thesis.

While these forms may be included in the document page count, their removal does not represent any loss of content from the thesis.
Conformément à la loi canadienne sur la protection de la vie privée, quelques formulaires secondaires ont été enlevés de cette thèse.

Bien que ces formulaires aient inclus dans la pagination, il n'y aura aucun contenu manquant. 


\begin{abstract}
The emergence of the Model-Driven Architecture (MDA) has changed the focus of software development from code to models, and raised the need for model transformations. The objective of this thesis is to develop a transformation of a UML model extended with MARTE performance annotations in to a more abstract performance model, which can then be used for the analysis of real-time properties. Up until now, the main method of implementing this kind of transformation has been through general-purpose languages such as Java. But this approach is not entirely consistent with the goals of MDA for reasons that will be further discussed.
\end{abstract}

The goal of this thesis is to use a special-purpose model transformation language to transform UML 2.1 Sequence Diagrams (with applied MARTE stereotypes) to Core Scenario Models. This transformation represents a task from a larger project entitled Performance by Unified Model Analysis (PUMA). The transformation language employed in this study is the ATL language by the ATLAS Group. As a hybrid between declarative and imperative rules, ATL is a fairly distinct language from general-purpose object-oriented languages. However, the use of this language reveals many advantages over the alternative approach, as will be demonstrated. Be that as it may, neither ATL nor other rule-based transformation languages like MOF-QVT have been around that long, and so conventional wisdom on their use remains narrow. One of the areas further explored in this thesis is the development of design strategies and patterns for model transformations. 


\begin{abstract}
The emergence of the Model-Driven Architecture (MDA) has changed the focus of software development from code to models, and raised the need for model transformations. The objective of this thesis is to develop a transformation of a UML model extended with MARTE performance annotations in to a more abstract performance model, which can then be used for the analysis of real-time properties. Up until now, the main method of implementing this kind of transformation has been through general-purpose languages such as Java. But this approach is not entirely consistent with the goals of MDA for reasons that will be further discussed.
\end{abstract}

The goal of this thesis is to use a special-purpose model transformation language to transform UML 2.1 Sequence Diagrams (with applied MARTE stereotypes) to Core Scenario Models. This transformation represents a task from a larger project entitled Performance by Unified Model Analysis (PUMA). The transformation language employed in this study is the ATL language by the ATLAS Group. As a hybrid between declarative and imperative rules, ATL is a fairly distinct language from general-purpose object-oriented languages. However, the use of this language reveals many advantages over the alternative approach, as will be demonstrated. Be that as it may, neither ATL nor other rule-based transformation languages like MOF-QVT have been around that long, and so conventional wisdom on their use remains narrow. One of the areas further explored in this thesis is the development of design strategies and patterns for model transformations. 
Table of Contents

LIST OF FIGURES ...................................................................................................................

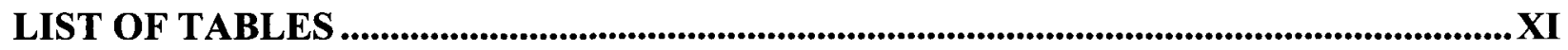

LIST OF CODE FRAGMENTS..........................................................................................

ACRONYMS .......................................................................................................................

CHAPTER 1. INTRODUCTION ................................................................................ 1

1.1 Motivation and Objectives .................................................................................................. 1

1.2 Research Scope of Thesis ................................................................................................... 3

1.3 Thesis Contributions ............................................................................................................... 5

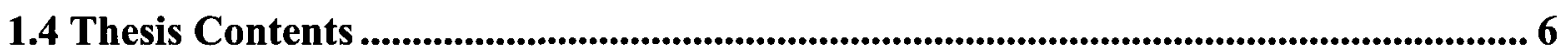

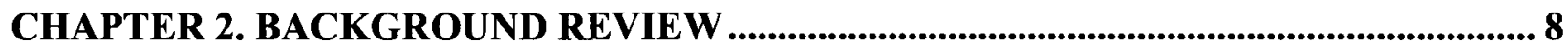

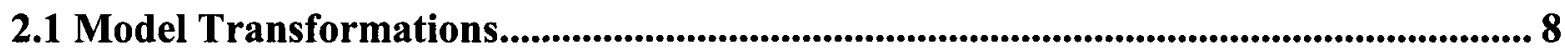

2.2 Metamodels .......................................................................................................................... 9

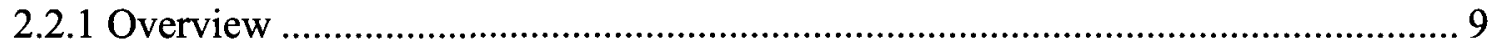

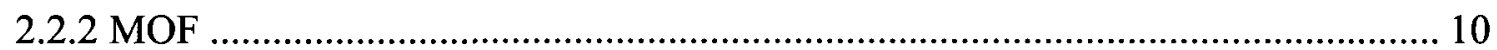

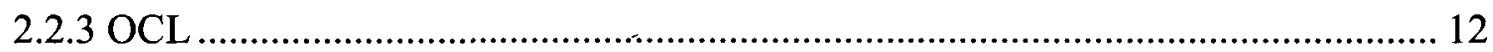

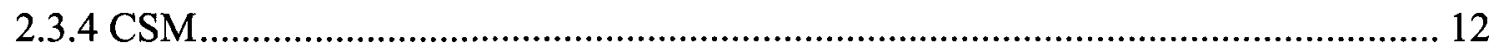

2.3 UML Profiles ......................................................................................................................................... 15

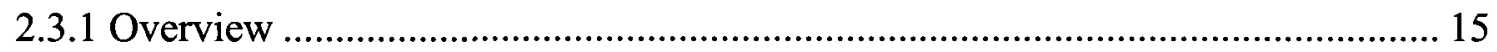

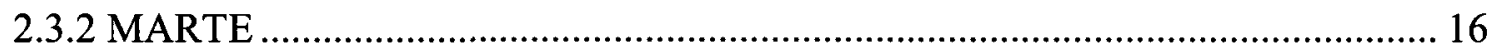

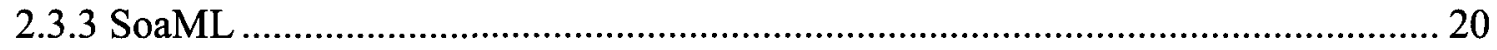

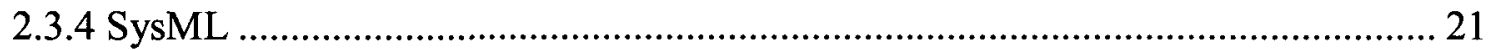

2.4 Modeling Tools and Transformation Languages................................................................ 21

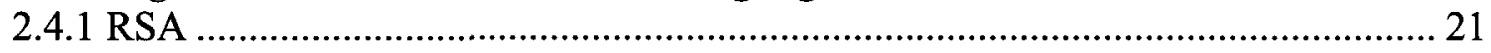

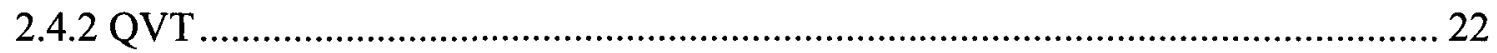

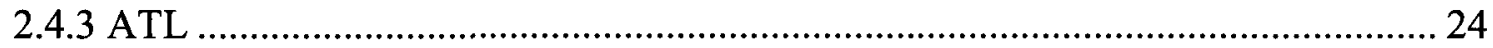

2.5 Methodologies For Model Transformations .................................................................. 29

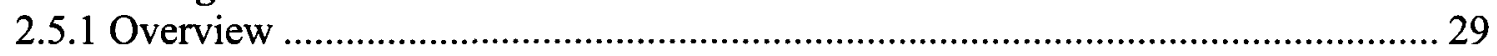

2.5.2 Transformations By Example …………………................................................ 30

2.5.3 Design Patterns .......................................................................................................... 32

CHAPTER 3. PROPOSED TRANSFORMATION APPROACH........................................34

3.1 Source Model................................................................................................................................ 34

3.2 Source to Target Mapping ................................................................................................... 38

3.3 Transformation Rules .............................................................................................................. 42

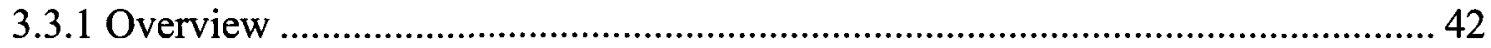

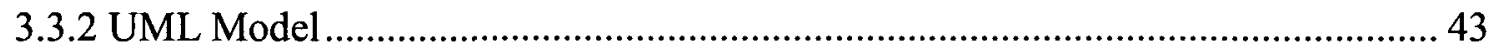

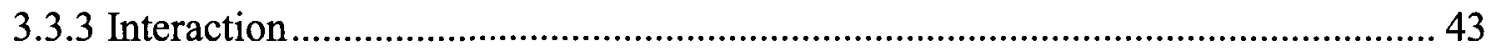

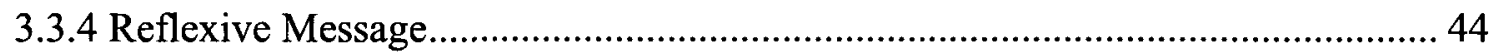

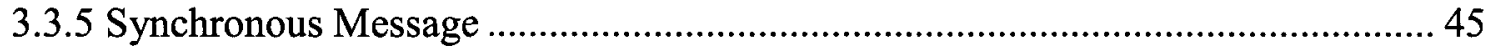

3.3.6 Asynchronous Message ................................................................................. 47 
3.3.7 Alternate Fragment ........................................................................................... 48

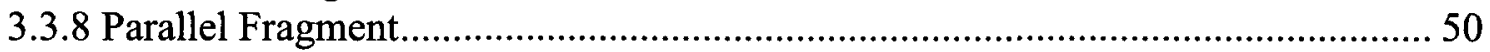

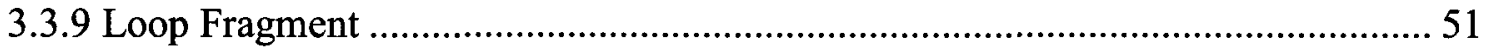

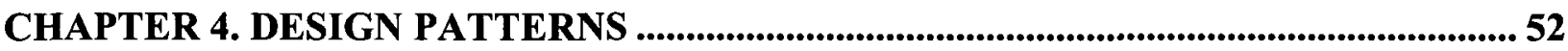

4.1 Two-tier Rule Approach ................................................................................................................ 52

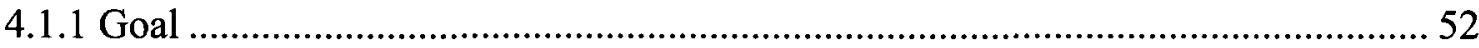

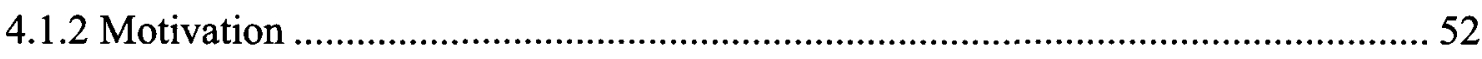

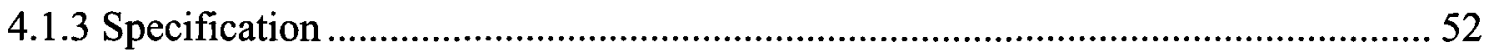

4.1.4 ATL Solution................................................................................................ 53

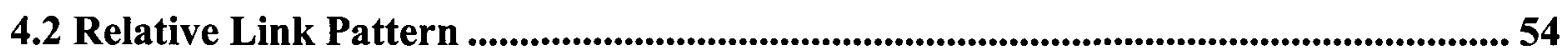

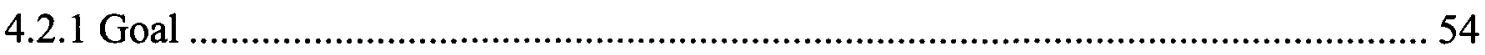

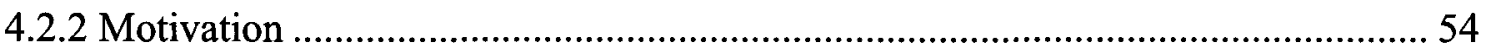

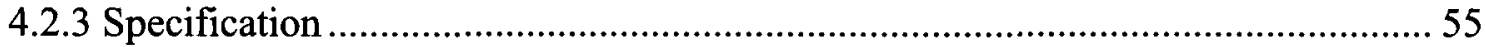

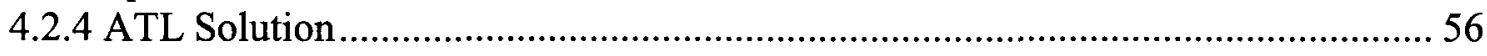

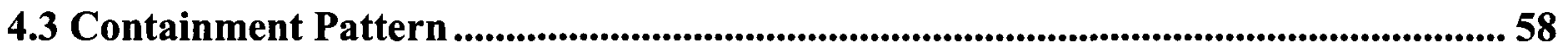

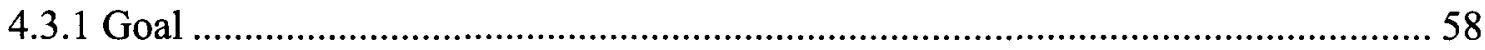

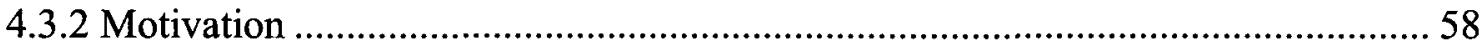

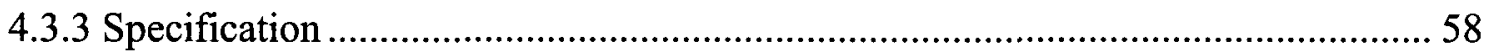

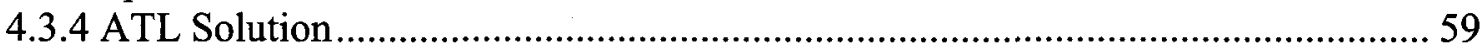

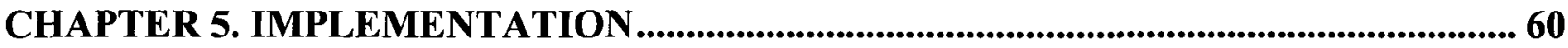

5.1 Transformation Model ...................................................................................... 60

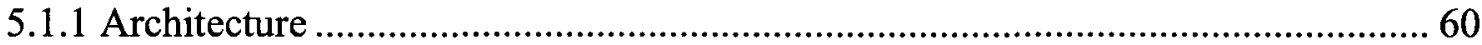

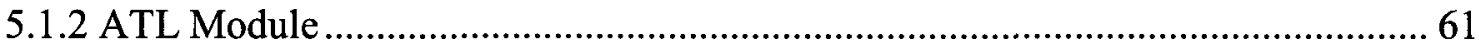

5.2 Transformation Process ....................................................................................................... 66

5.3 Output Visualization ........................................................................................................... 68

CHAPTER 6. VERIFICATION AND CASE STUDY ….................................................. 73

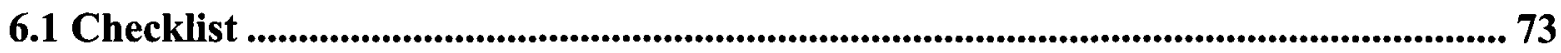

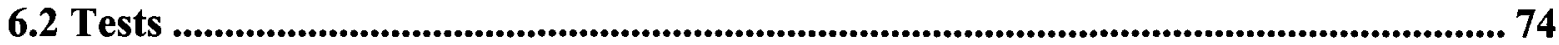

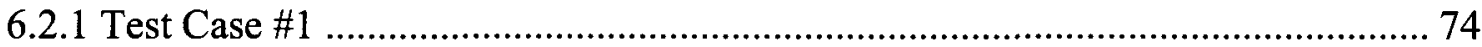

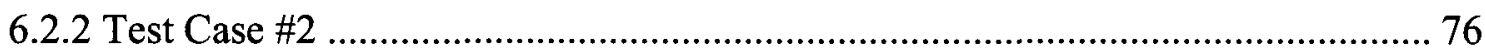

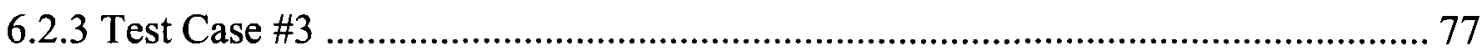

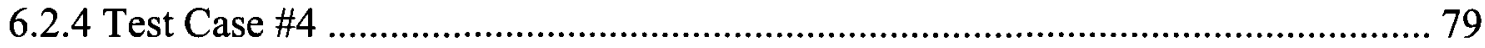

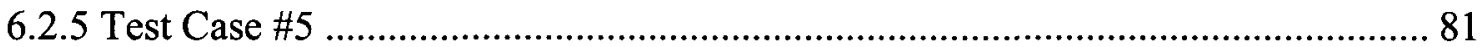

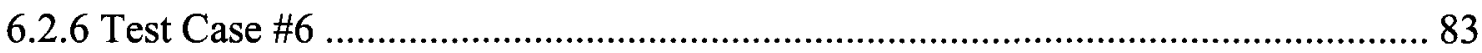

6.2.7 PUMA Example Test Case ................................................................................... 85

6.3 Case Study: Video Acquisition Scenario ................................................................................... 88

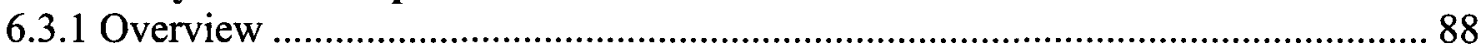

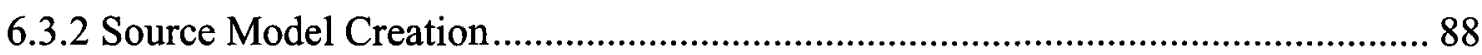

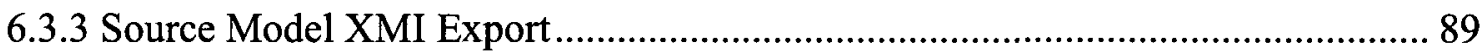

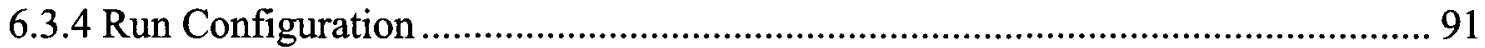

6.3.5 Transformation Execution ..................................................................................... 93

6.3.6 CSM Visualizer Execution .................................................................................. 94

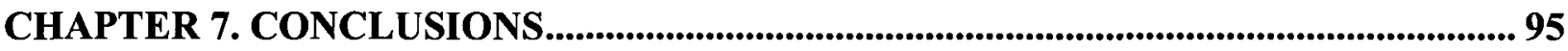

7.1 Accomplishments ............................................................................................................... 95 


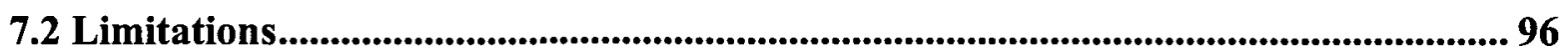

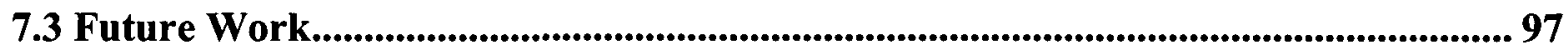

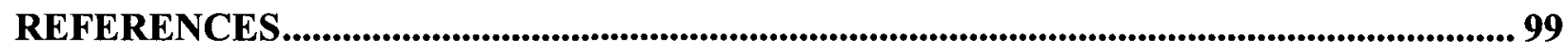

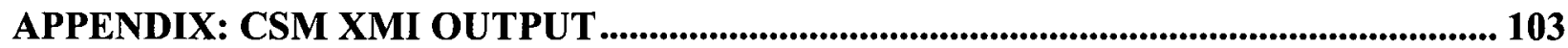

A.1 Example Interaction ...................................................................................................................... 103

A.2 Test Case \#1 ............................................................................................................................................... 104

A.3 Test Case \#2 ..................................................................................................................... 105

A.4 Test Case \#3 .......................................................................................................................................... 107

A.5 Test Case \#4 ................................................................................................................ 109

A.6 Test Case \#5 ...................................................................................................................................... 109

A.7 Test Case \#6 ..................................................................................................................... 112

A.8 PUMA Example .................................................................................................................... 113

A.9 Video Store ................................................................................................................................. 115 


\section{List of Figures}

FIGURE 1-1: UML+MARTE INTERACTION DIAGRAM TO CSM TRANSFORMATION PROCESS

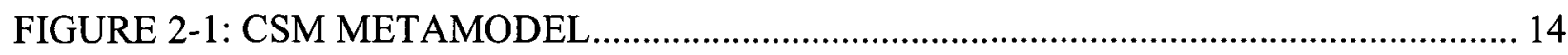

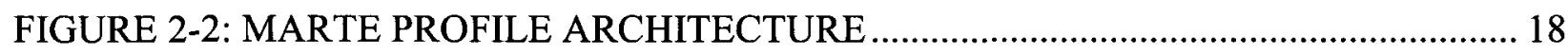

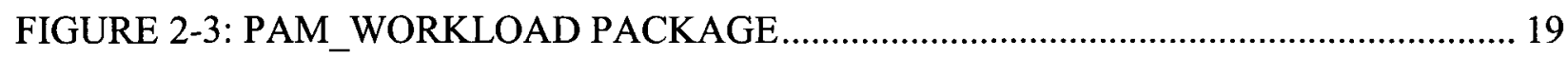

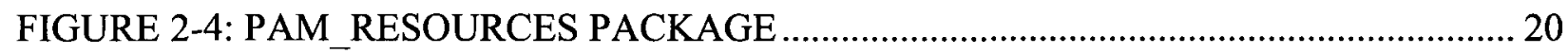

FIGURE 2-5: ATL TRANSFORMATION EXAMPLE - METAMODELS …………………...... 25

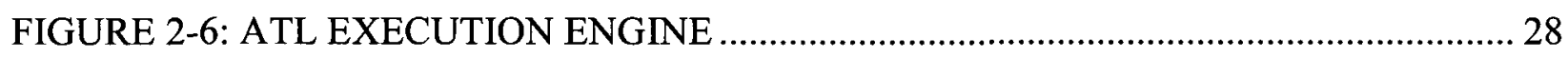

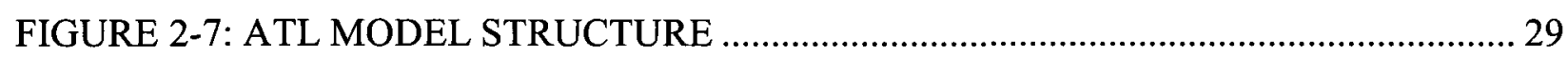

FIGURE 2-8: MODEL TRANSFORMATION BY EXAMPLE PROCESS OVERVIEW .........31

FIGURE 3-1: PARTIAL UML2 SEQUENCE DIAGRAM METAMODEL ................................ 35

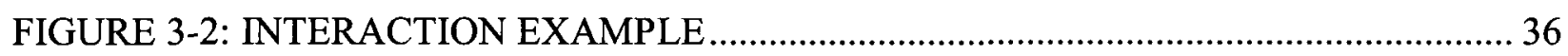

FIGURE 3-3: OBJECT DIAGRAM SHOWING THE MODEL ELEMENTS OF THE

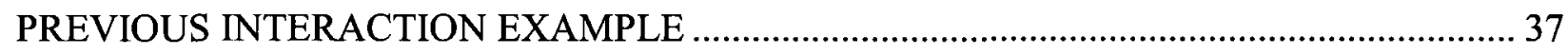

FIGURE 3-4: UML MODEL - TRANSFORMATION RULE ............................................... 43

FIGURE 3-5: INTERACTION - TRANSFORMATION RULE ................................................ 43

FIGURE 3-6: REFLEXIVE MESSAGE - TRANSFORMATION RULE.....................................4 44

FIGURE 3-7: REFLEXIVE MESSAGE - TRANSFORMATION EXAMPLE ............................ 44

FIGURE 3-8: SYNCHRONOUS MESSAGE - TRANSFORMATION RULE ............................ 45

FIGURE 3-9: REPLY MESSAGE - TRANSFORMATION RULE .......................................... 46

FIGURE 3-10: SYNCHRONOUS/REPLY MESSAGES - TRANSFORMATION EXAMPLE 46

FIGURE 3-11: ASYNCHRONOUS MESSAGE - TRANSFORMATION RULE ...................... 47

FIGURE 3-12: ASYNCHRONOUS MESSAGE - TRANSFORMATION EXAMPLE .............. 48

FIGURE 3-13: ALTERNATE FRAGMENT - TRANSFORMATION RULE ………………..... 49

FIGURE 3-14: ALTERNATIVE FRAGMENT - TRANSFORMATION EXAMPLE................ 49

FIGURE 3-15: PARALLEL FRAGMENT - TRANSFORMATION RULE................................50

FIGURE 3-16: PARALLEL FRAGMENT - TRANSFORMATION EXAMPLE ........................50 
FIGURE 3-17: LOOP FRAGMENT - TRANSFORMATION RULE..................................... 51

FIGURE 3-18: LOOP FRAGMENT - TRANSFORMATION EXAMPLE ................................ 51

FIGURE 4-1: TWO-TIER RULE - GENERIC TRANSFORMATION STRUCTURE............... 53

FIGURE 4-2: TWO-TIER RULE - ATL TRANSFORMATION STRUCTURE........................54

FIGURE 4-3: RELATIVE RELATIONSHIPS OF SOURCE AND TARGET MODELS ......... 55

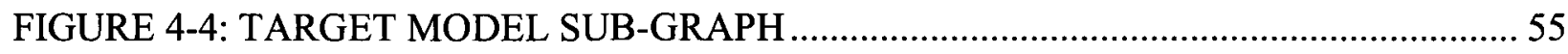

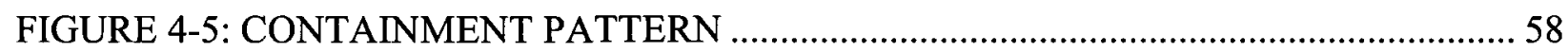

FIGURE 5-1: UML+MARTE INTERACTION DIAGRAM TO CSM ATL MODEL................... 60

FIGURE 5-2: MATCHED RULE DOMAIN OF MM2CSM ……………………...................... 62

FIGURE 5-3: USER STEPS IN TRANSFORMATION PROCESS ..........................................67

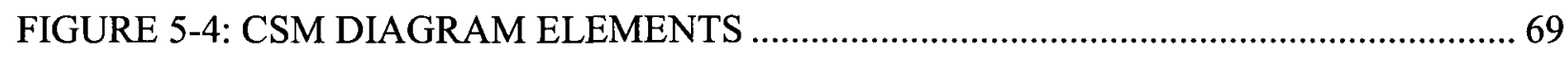

FIGURE 5-5: CSM VISUALIZER JAVA CLASS DIAGRAM............................................... 72

FIGURE 6-1: TEST CASE \#1 - SOURCE MODEL................................................................. 74

FIGURE 6-2: TEST CASE \#1 - TARGET MODEL.................................................................... 75

FIGURE 6-3: TEST CASE \#2 - SOURCE MODEL_............................................................... 76

FIGURE 6-4: TEST CASE \#2 - TARGET MODEL ................................................................. 77

FIGURE 6-5: TEST CASE \#3 - SOURCE MODEL ..................................................................... 77

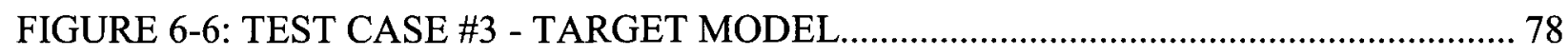

FIGURE 6-7: TEST CASE \#4 - SOURCE MODEL_.................................................................... 79

FIGURE 6-8: TEST CASE \#4 - TARGET MODEL ...................................................................... 80

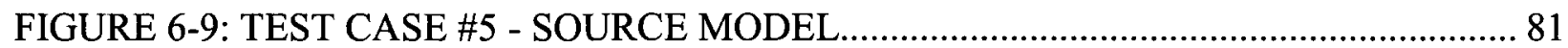

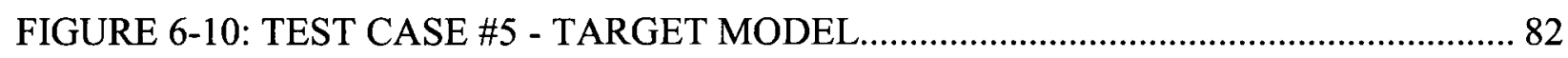

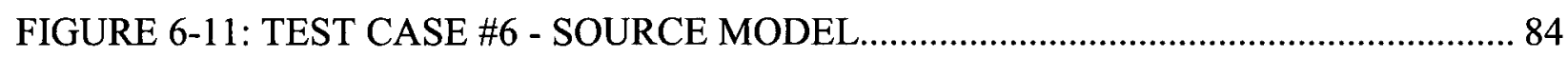

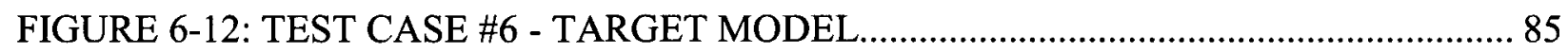

FIGURE 6-13: PUMA EXAMPLE TEST CASE - SOURCE MODEL ........................................ 86

FIGURE 6-14: PUMA EXAMPLE TEST CASE - TARGET MODEL ……................................. 87

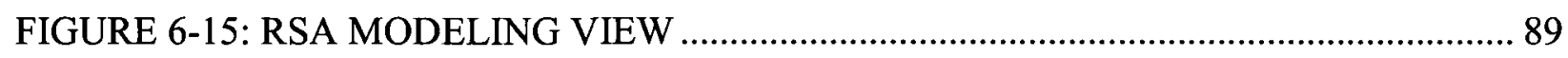

FIGURE 6-16: SOURCE MODEL XMI EXPORT DIALOG - $1^{\text {ST }}$ SCREEN ……...................... 90 
FIGURE 6-17: SOURCE MODEL XMI EXPORT DIALOG - $2^{\text {ND }}$ SCREEN ...................... 91

FIGURE 6-18: ATL TRANSFORMATION RUN CONFIGURATION...............................92

FIGURE 6-19: CSM VISUALIZER RUN CONFIGURATION ....................................... 93

FIGURE 6-20: CSM VISUALIZER EXECUTION .................................................... 94 


\section{List of Tables}

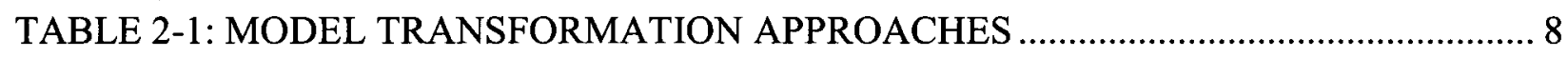

TABLE 2-2: FOUR-LAYER METAMODEL HIERARCHY ................................................... 10

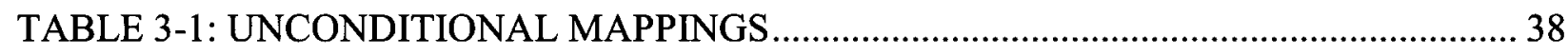

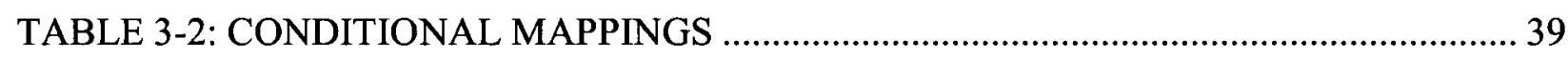

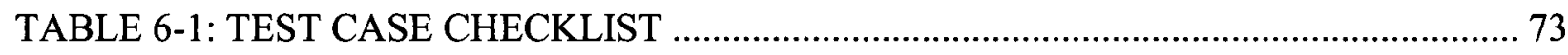




\section{List of Code Fragments}

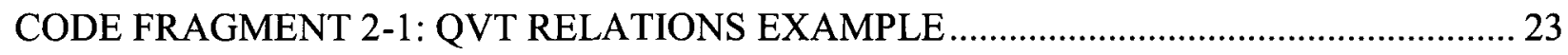

CODE FRAGMENT 2-2: ATL TRANSFORMATION EXAMPLE - MODULE ......................... 26

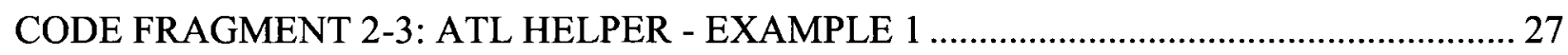

CODE FRAGMENT 2-4: ATL HELPER - EXAMPLE 2 …………....................................... 27

CODE FRAGMENT 4-1: ATL ALGORITHM FOR RELATIVE LINK PATTERN.................. 57

CODE FRAGMENT 4-2: ATL IMPLEMENTATION OF CONTAINMENT PATTERN ........ 59

CODE FRAGMENT 4-3: XMI OF TARGET MODEL FOLLOWING APPLICATION OF

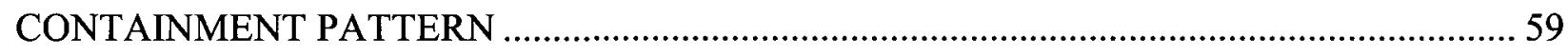

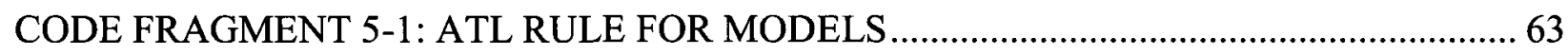

CODE FRAGMENT 5-2: ATL HELPER FOR GETTING PREVIOUS MESSAGE .................. 64

CODE FRAGMENT 5-3: ATL RULE FOR SYNCHRONOUS MESSAGES ...........................64

CODE FRAGMENT 5-4: ALGORITHM FOR CONSTRUCTING CSM DIAGRAM .............. 70 


\section{Acronyms}

\begin{tabular}{|c|c|}
\hline ATL & Atlas Transformation Language \\
\hline BFS & Breadth-First Search \\
\hline $\mathrm{CCM}$ & CORBA Component Model \\
\hline CORBA & Common Object Request Broker Architecture \\
\hline CSM & Core Scenario Model \\
\hline ECORE & EMF Core meta-metamodel \\
\hline EMF & Eclipse Modeling Framework \\
\hline GQAM & Generic Quantitative Analysis Modeling \\
\hline IDE & Integrated Development Environment \\
\hline MARTE & Modeling and Analysis of Real-Time and Embedded systems \\
\hline MDA & Model Driven Architecture \\
\hline MDD & Model Driven Development \\
\hline MOF & Meta-Object Facility \\
\hline NFP & Non-Functional Property \\
\hline OCL & Object Constraint Language \\
\hline
\end{tabular}




$\begin{array}{ll}\text { OMG } & \text { Object Management Group } \\ \text { QVT } & \text { Query/View/Transformations } \\ \text { PAM } & \text { Performance Analysis Modeling } \\ \text { PIM } & \text { Platform-Independent Model } \\ \text { PSM } & \text { Platform-Specific Model } \\ \text { PUMA } & \text { Performance by Unified Model Analysis } \\ \text { RDBMS } & \text { Relational Database Management System } \\ \text { ReST } & \text { Representational State Transfer } \\ \text { RSA } & \text { Rational Software Architect } \\ \text { RTES } & \text { Real-Time Embedded Systems } \\ \text { XMI } & \text { XML Metadata Interchange } \\ \text { SOA } & \text { Service Oriented Architecture } \\ \text { SPT } & \text { Schedulability, Performance and Time } \\ \text { UML } & \text { Unified Modeling Language } \\ & \\ \text { XML } & \end{array}$




\section{Chapter 1. Introduction}

\section{$\underline{1.1 \text { Motivation and Objectives }}$}

OMG's Model Driven Architecture (MDA) has emerged as a new paradigm for the software development process by raising the level of abstraction of models to allow for the automatic generation of code from models [MDA]. The goals of MDA are as follows: improve understanding of problems, minimize development risks, improve communication between stakeholders, increase focus on the domain problems rather than their implementations, and improve both interoperability and portability of software systems [SV+06]. By realizing these

goals, gains can be made in development speed, productivity, software quality, and reusability.

The central idea behind MDA is that there exist different levels of abstraction of modeling. There are Platform-Independent Models (PIMs), which describe the domain-related specifications, and Platform-Specific Models (PSMs), which describe the implementation of a PIM on different platforms. PSMs are generated from PIMs, through model-to-model transformations, and contain specific concepts of the target platform [SV+06]. From PSMs, model-to-code transformations produce platform-specific code based on code templates of the given platform. However, the development of software-intensive systems through the transformation of models to models, and then to executable components and applications, requires a standard way to express transformations [Miller+04].

By changing the focus of software development from code to models, MDA enable us to approach the verification of non-functional properties of software (such as performance, reliability, security, etc.) by transforming UML design specifications annotated with extra information corresponding to the property to be verified into appropriate analysis models. Over 
the years, many modeling formalisms, techniques and tools have been developed for the analysis of different non-functional properties (e.g., Markov chains, queueing networks, Petri nets, fault trees, reliability block diagrams, formal logic models, process algebras, simulation, etc). The research challenge is to bridge the gap between MDA-based software development tools and different existing analysis tools by using model transformations. The non-functional property of interest in this thesis is software performance. The thesis addresses the problem of automatic derivation of a performance model from a UML software model with performance annotations, as presented in section 1.2 .

Up until recently, many of the approaches to model transformations have been based on general-purpose programming languages, such as Java [CGM+08]. While the use of the Direct Manipulation paradigm can satisfy all the functional requirements of a transformation model, there is no way to formally verify that the target model is consistent with the source model that it was generated from. There are many possible paradigms that can be used to transform models in addition to Direct Manipulation including Relational, Graph-based transformations, Structure Driven, and Hybrid approaches (as discussed in Chapter 2). OMG has released a StructureDriven standard, MOF 2.0 QVT, for the specification of transformation models. Based on this standard, the ATLAS INRIA \& LINA Group released the Atlas Transformation Language (ATL) framework. Both of these languages can be classified as rule-based transformation languages.

ATL offers semantics that allow the specification of transformation rules. This offers several advantages over the Direct Manipulation approach described above. ATL transformations are much more easily maintained than the previously mentioned approach because rules can be added, removed or modified without effecting the implementation aspects, such as rule scheduling or composition [CGM+08]. These aspects are managed by the ATL 
engine, as opposed to transformation developers in Java who must explicitly address these aspects within their code. ATL also benefits from having a structure that can be understood much more easily among developers of different disciplines within a team, as opposed to Java where the transformation logic is more difficult to retrieve since it's deeply embedded within the code. Lastly and perhaps the most pertinent advantage of ATL is that it opens up the possibility of using formal proofs to verify transformation correctness through ATL's rule traceability capability. Formal proofs are not feasible with transformation models written in general purpose programming languages [CGM+08].

Being a relatively young language, transformation development in ATL has a longer start-up time than development in general-purpose languages because the education, time, and skills necessary to properly utilize ATL are not as common as the latter. Also, the ATL language requires the definition and maintenance of source and target metamodels, of which the transformation model is tightly coupled to. These challenges have given rise to research in developing design methodologies targeted to ATL and other rule-based transformation languages.

\subsection{Research Scope of Thesis}

The research context of this thesis is the Performance by Unified Model Analysis (PUMA) architecture, which proposes to annotate UML design specifications with quantitative performance attributes for the purposes of analysis, as described in the paper "Methods and Tools for Performance by Unified Model Analysis (PUMA)" [WPPSIM+05]. At the paper's time of writing, a UML Profile for Schedulability, Performance and Time (SPT) defined for UML 1.X was used for performance annotations [SPT]. For the purposes of non-functional analyses, 
PUMA attempted to unify different kinds of performance models generated from UML extended with SPT annotations.

The PUMA architecture transformed UML+SPT models in to an intermediate model named Core Scenario Model (CSM), which abstracted the design information relevant to performance analysis. CSM presents a visual model much simplified from its UML origins, where performance verification is far less difficult and error-prone. However, since the publishing of the PUMA paper, OMG has produced another UML Profile for Modeling and Analysis of Real-time and Embedded Systems (MARTE) defined for UML 2.X, that replaces SPT. In this thesis, the ground work laid out in the PUMA project is continued by transforming UML models extended with MARTE stereotypes in to CSMs.

Figure 1-1 shows the entire transformation process of UML+MARTE Interaction Diagrams to CSMs. The main modeling tool used for producing UML Sequence Diagrams is the visual diagram tool of Rational Software Architect. Additionally, a third party plugin that adds MARTE stereotypes to the RSA modeling environment is available [MARTEPlugin]. Produced UML models can then be serialized in XML Metadata Interchange (XMI) form using an export feature available in RSA. The entire transformation sub-process takes place within the Eclipse environment, whereby the source file is read by Eclipse's internal reader. After parsing the file, a virtual UML+MARTE is constructed using the Eclipse Modeling Framework (EMF) library, which is interoperable with the ATL Transformation Model. After accepting the source model and executing the transformation, the ATL Engine produces a virtual CSM specified in the EMF Core meta-metamodel (ECORE), which in turn gets serialized by Eclipse's internal XMI writer. The target file can then be used for analysis by performance domain utilities. 


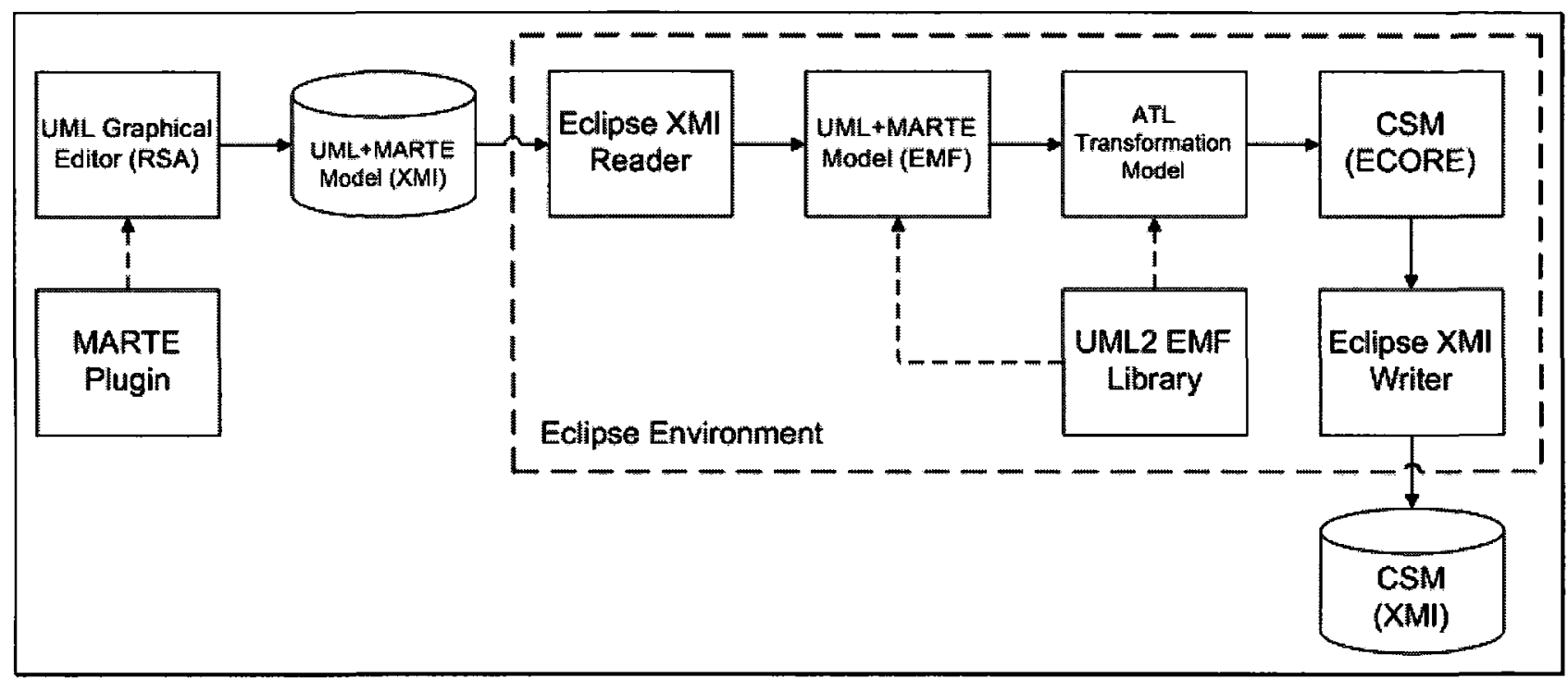

Figure 1-1: UML+MARTE Interaction Diagram to CSM Transformation Process

\subsection{Thesis Contributions}

The goal of this thesis is to define and implement an ATL transformation model of UML sequence diagrams (with MARTE applied stereotypes) to CSMs. The necessary steps for achieving this goal have resulted in the following contributions:

- Transformation Mappings - The design of mapping rules of UML+MARTE elements to CSM elements through a thorough analysis of the problem domain. Furthermore, since these mappings are platform-independent, they form the basis for similar transformation models using tools other than ATL.

- Model Transformation Design Patterns - Design patterns describe solutions to common software design problems arising within a certain context; patterns generally codify expert knowledge and "best practices" and facilitate reuse of successful designs [GHJV+95]. Model transformation design patterns are targeted toward the development of model transformations. In the thesis, a number of model transformation design patterns 
are formulated as a contribution to knowledge, based on the experience gained from developing the ATL transformation.

- ATL Transformation Implementation - The transformation mappings and transformation model design patterns of this study are implemented in an ATL project, which showcases the methodology explored in this thesis and forms the basis for future work. The test cases used to verify the implementation are also described.

- CSM Visualizer - Because of the XMI storage facility of the target model produced by ATL, this study benefited from the development of a CSM Visualizer utility. This utility produces a graphical representation of the CSMs generated by the ATL transformation model, making it easier to perform a human visual verification of the target model.

\subsection{Thesis Contents}

This thesis consists of 7 chapters, including this introductory chapter, and an appendix. Each chapter is broken down in to separate sections and subsections.

Chapter 2 gives background material on transformation models, which includes: descriptions of the different approaches, language specifications of the models, tools used to bring about the design and transformation of the models, and finally, methodologies used to design transformation models.

The 3rd chapter deals with the specific domain problem of this study, which is the transformation of UML+MARTE models to CSMs. Given here are detailed descriptions of the metamodels of the source and target models, an in-depth look at the structure of model instances, and the main mappings between the source and target models. 
Chapter 4 describes the transformation design patterns derived and employed in this study. Each pattern is described in full, with an analysis of each of their platform-independent and ATL-specific approaches.

In chapter 5, a detailed analysis of the ATL implementation of UML+MARTE models to CSMs is made. Through each of the chapter sections, insights in to the transformation architecture, its process, and the development of CSM Visualizer, will be gained.

Chapter 6 presents a requirements checklist, several test cases to perform trials on, and a case study which presents all the necessary steps to execute the model transformation of this study (from start to finish).

The 7 th and final chapter of this study is the conclusion. Here, a section detailing the accomplishments of the work performed in this study will be presented, along with sections discussing the study's limitations and possible areas for future work.

The appendix contains the full XML output of the target models produced by the model transformation of this study. 


\section{Chapter 2. Background Review}

\section{$\underline{\text { 2.1 Model Transformations }}$}

A model transformation is the process of converting one model to another model of the same system [MDA]. Ultimately, this means converting a source model in to a target model, both of which conform to their respective metamodels [BJP+05]. Furthermore, these metamodels are based on a common meta-metamodel. Each transformation is based around a set of rules, where a relationship is formed between a source element and target element(s).

Model transformations can be implemented using many different programming paradigms. Logic programming with its unification-based matching, search, and backtracking seems a natural choice to implement the relational approach, where predicates can be used to describe the relations $[\mathrm{CH}+03]$.

Table 2-1 details the multiple approaches available for model-to-model transformations [CH+03]:

\begin{tabular}{|l|l|l|}
\hline \multicolumn{1}{|c|}{$\begin{array}{c}\text { Transformation } \\
\text { Approach }\end{array}$} & \multicolumn{1}{c|}{ Description } & \multicolumn{1}{c|}{ Examples } \\
\hline Direct Manipulation & $\begin{array}{l}\text {-usually implemented as an object-oriented } \\
\text { framework with minimal infrastructure to } \\
\text { organize the transformations }\end{array}$ & Jamda, JMI, Java \\
\hline Relational & $\begin{array}{l}\text {-involves specifying relations between } \\
\text { source and target elements using declarative } \\
\text { constraints and implementing the } \\
\text { transformation through executable semantics }\end{array}$ & $\begin{array}{l}\text { QVT and related } \\
\text { technologies }\end{array}$ \\
\hline
\end{tabular}

Table 2-1: Model Transformation Approaches 


\begin{tabular}{|l|l|l|}
\hline $\begin{array}{l}\text { Graph-Transformation- } \\
\text { Based }\end{array}$ & $\begin{array}{l}\text {-consists of transformation rules that map a } \\
\text { left-hand side graph pattern to a right-hand } \\
\text { side graph pattern }\end{array}$ & $\begin{array}{l}\text { VIATRA, ATOM, } \\
\text { GreAT, UMLX, and } \\
\text { BOTL }\end{array}$ \\
\hline Structure-Driven & $\begin{array}{l}\text {-rule based methodology with two distinct } \\
\text { phases: creating the hierarchical structure of } \\
\text { the target model; setting attributes and } \\
\text { references in the target. }\end{array}$ & OPT, OptimalJ \\
\hline Hybrid & $\begin{array}{l}\text {-is a composition of declarative and } \\
\text { imperative approaches. } \\
\text {-in addition to mapping rules, operation rules } \\
\text { exist as executable transformation rules. }\end{array}$ & ATL, XDE \\
\hline
\end{tabular}

Table 2-1: Model Transformation Approaches (cont'd)

It is possible for an application developer to come up with the logic necessary to design the transformation based on a few examples. However, it becomes increasing difficult to develop a model transformation that converts all possible source models in to a target model. This is especially true in more complicated transformations. For many developers, an incomplete transformation model may be sufficient given the application requirements, sparing them much time and resources over building a complete transformation model [SLSS+08]. Typically, transformation development occurs incrementally, covering each requirement on a step-by-step basis.

\section{$\underline{\text { 2.2 Metamodels }}$}

\subsubsection{Overview}

A metamodel is a model that defines the abstract syntax and static semantics of a modeling language. This includes the modeling language's constructs, relationships and 
constraints. The concrete syntaxes (ex. visual, textual) of a modeling language are defined separately from the metamodel. In turn, specifications of metamodels requires their own metamodeling language, known as a meta-metamodel. Officially, OMG has specified the Metaobject Facility (MOF) for this purpose, although alternate meta-metamodels do exist (like ECORE for the Eclipse environment).

OMG has specified a four layer hierarchy to metamodeling [UML2Infra]. This hierarchy consists of the following:

\begin{tabular}{|l|l|l|}
\hline Layer & \multicolumn{1}{|c|}{ Description } & \multicolumn{1}{|c|}{ Example } \\
\hline M3 & $\begin{array}{l}\text { Meta-metamodels define languages for specifying metamodels, } \\
\text { preferably ones sharing common design philosophies and } \\
\text { constructs. }\end{array}$ & MOF, ECORE \\
\hline M2 & $\begin{array}{l}\text { Metamodels are more elaborate, they define languages for } \\
\text { specifying models. }\end{array}$ & UML2 \\
\hline M1 & $\begin{array}{l}\text { Models define languages that describe semantic domains such } \\
\text { as software, business, processes and requirements. }\end{array}$ & $\begin{array}{l}\text { UML2 Class/State/ } \\
\text { Sequence Diagrams }\end{array}$ \\
\hline M0 & $\begin{array}{l}\text { This layer contains run-time instances of model elements } \\
\text { defined in M1. }\end{array}$ & Java Thread \\
\hline
\end{tabular}

Table 2-2: Four-layer Metamodel Hierarchy

\subsubsection{MOF}

The internet is composed of many applications and data sources that rely on transparent data exchange [MOF]. Applications, databases, middleware, and other software all use metadata to describe the data that can be exchanged. However, the incompatibilities that exist between the different proprietary metadata used by each system remain a central challenge to data exchange. A common metadata framework could benefit applications that rely heavily on data exchange 
such as data warehousing, business intelligence, e-commerce, and information portals [MOF]. In order to address this concern, the OMG released the Meta Object Facility (MOF), which in addition to providing a common metadata framework, includes metadata services that enable the development and interoperability of model and metadata driven systems [MOF].

In order to maintain interoperability with other OMG standards and provide a platform independent solution, MOF 2.0 reuses the UML2 Infrastructure library. Standard class modeling concepts such as importing, classes, generalizations and associations all exist in MOF 2.0. This framework also includes packages that define new extensions for addressing metadata management concerns. They introduce: Reflections, which allow models to be self describing; Identifiers, which uniquely identify metamodel objects without relying on model data that may be subject to change; and finally Extensions, which can be used to extend model elements with name/value pairs [MOF].

MOF has two variants: Essential MOF (EMOF) and Complete MOF (CMOF). EMOF is a straightforward framework for mapping MOF models to implementations such as XMI or Java [MOF]. By allowing metamodels to be defined using simple concepts, EMOF provides a low barrier entry for use of its metadata facilities. The meta-metamodel employed by the Eclipse environment, is based on EMOF [Eclipse]. The other variant, CMOF, provides extended capabilities over the EMOF package, which allow the definition of more complex metamodels, like the UML2.0 framework.

The XML Metadata Interchange (XMI) standard allows MOF metadata to be exchanged in XML form. This also provides the benefit of XML validation, which verifies that a model conforms to a metamodel, at least as far as structure since some of the model's semantic 
constraints can't be expressed in XMI form. Each XMI document conforms to an XMI model, which is an instance of MOF for describing XMI-specific information in the document such as versions, extensions, and differences [XMI]. XMI models enable both: XMI document metadata to be treated as MOF metadata, and standard MOF APIs to access and construct XMI elements as MOF objects.

\subsubsection{OCL}

Highly abstract models, like UML class diagrams, are typically not refined enough to provide specifications for constraints, which are often described using natural languages [OCL]. A formal language offers a less ambiguous way of defining constraints, however they have the disadvantage of being more difficult to understand by someone who does not have a strong mathematical background. As a result of this shortcoming, OMG introduced the Object Constraint Language (OCL), a formal language that can describe expressions on UML models.

The OCL specification is based on the core libraries of MOF and UML, although taken as a whole, it can only be used with the latter [OCL]. Since OCL is a declarative language, its expressions do not change anything in the models they describe, they only return a value. Hence, OCL applications include: use as query languages, describing guards, specifying operation constraints, describing pre/post conditions on operations/methods, and many others uses [OCL].

\subsubsection{CSM}

The Core Scenario Model (CSM) is a metamodel for the building of performance models for a wide variety of applications, including telecommunications, business systems and web services $[\mathrm{PW}+06]$. Models of this type are usually generated from data extracted from UML 
models. The generated CSM then allows for a review of the consistency and completeness of the original model $[\mathrm{PW}+06]$.

CSM follows the SPT UML profile, in that it consists of a Scenario main class containing a series of Steps, each connected by PathConnections. PatchConnections have several subtypes including Forks, Joins, Decisions and Merges. Each step may also contain a sub-Scenario, containing its own Steps and PathConnections.

Every Scenario has a Workload specified which may be one of two types, open or closed. OpenWorkloads have arrivals from the environment, while ClosedWorkloads have a fixed number of requests that may be in the system $[\mathrm{PW}+06]$.

Steps may have one of two types of Resources associated with them, active or passive. ActiveResources spontaneously generate events, while passive Resources respond to requests $[\mathrm{PW}+06]$.

Figure 2-1 illustrates the metamodel of CSM. It should be noted that the CSM metamodel implemented in the transformation model of this study is identical to the one represented in the diagram, except that Start and End are subclasses of the Step class as defined in older specifications of CSM $[\mathrm{PW}+04]$. 


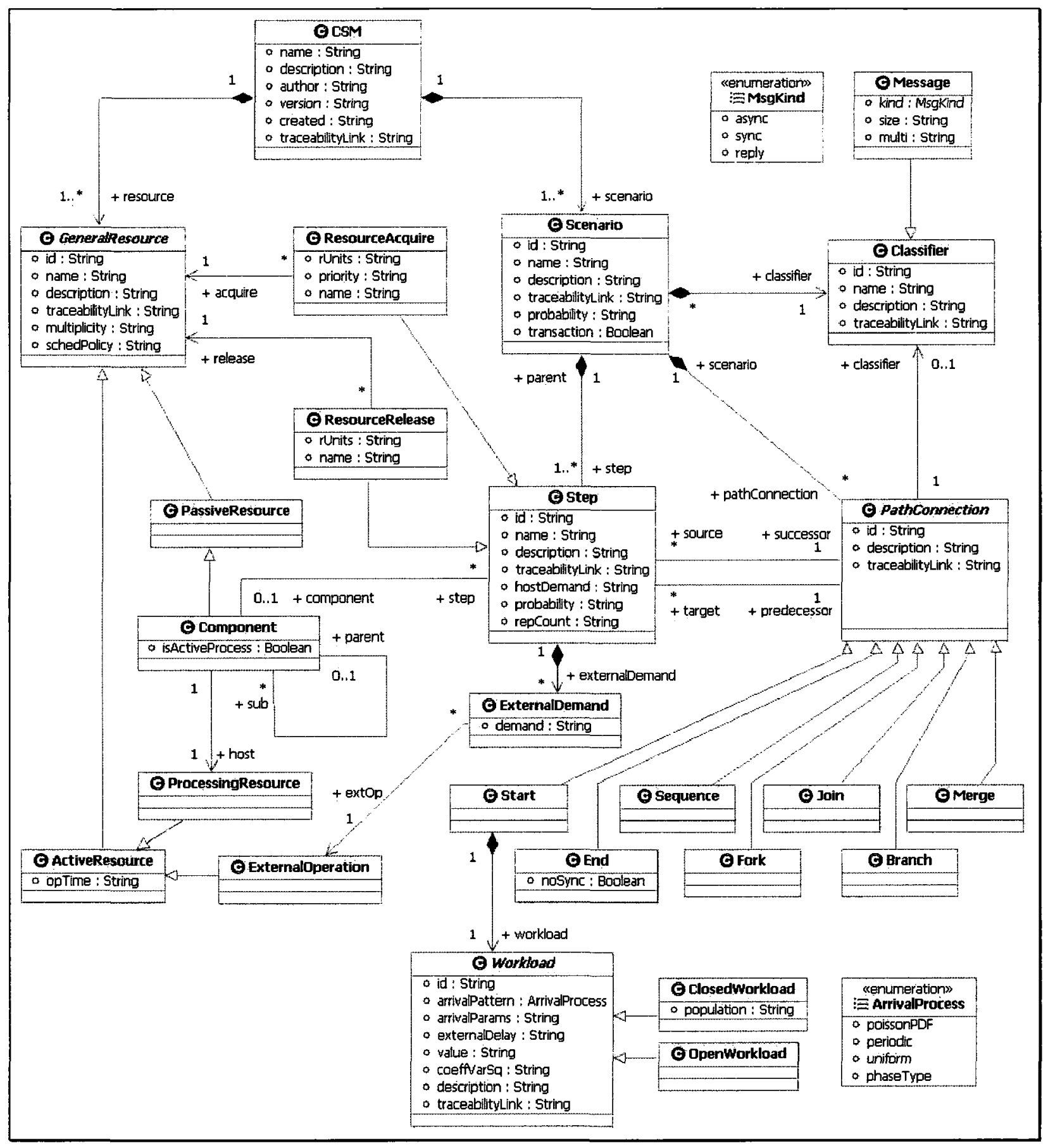

Figure 2-1: CSM Metamodel 


\subsection{UML Profiles}

\subsubsection{Overview}

A UML Profile is a package of related specializations of general UML elements that capture domain-specific concepts. A new dialect of UML can be defined by using Profiles to customize the language for particular platforms (e.g., J2EE/EJB, .NET/COM+) and domains (e.g., finance, telecommunications, aerospace) [UML2Infra].

Profiles forms one of two packages in the UML InfrastructureLibrary. The other package, Core, is a complete metamodel designed for high reusability, where other metamodels at the same metalevel either import or specialize its specified metaclasses [UML2Infra]. The Profiles package makes use of the abstract syntax and semantics defined in the Core package, making Profiles dependent upon it. Although Profiles are intended to be used with the UML metamodel, it's possible to use them with any metamodel that is based on the Core package [UML2Infra].

Profiles do not extend any existing UML metaclasses, nor do they add any new ones, however they do make use of standard extension mechanisms. These mechanisms are stereotypes, tagged values, and constraints, all of which apply to already existing metaclasses. These extension mechanisms are supported by tool vendors, allowing standard UML tools able to accept new profile definitions and usage without changes to the tools.

Of the two existing general approaches to extending UML, using profiles is considered to be a lightweight approach. A heavyweight approach involves modifying the UML metamodel by using MOF. An example of this is when it is required to add a new UML metaclass to the UML metamodel. The main disadvantage of the heavyweight approach is that the standard UML tools 
do not recognize the extensions, so the tools need to be modified as well. In the following subsections, a few UML profiles standardized by OMG are described.

\subsubsection{MARTE}

Since the adoption of the UML standard and its new advanced release UML2, the modeling language has been used in the development of a large number of time-critical and resource-critical systems [MARTE]. However, while a useful tool, a consensus emerged that UML lacked a few key features necessary for real-time and embedded systems (RTES) development. In particular, it was noticed that the lack of a quantifiable notion of time and resources was an impediment to its broader use in the real-time and embedded domain [MARTE]. Fortunately, the extensible nature of UML allowed the definition of a new standard, MARTE, without making use of heavyweight extensions.

MARTE is a UML Profile that provides support for specification, design, and verification/validation stages of RTES models [MARTE]. It is intended as a replacement for the UML SPT profile, of which MARTE borrows many constructs. SPT, which was defined for older UML versions (1.X), lacked a mechanism to extend or refine these constructs for more specific needs, hence the formulation of a new UML Profile for UML 2.X versions was necessary [MARTE].

MARTE defines foundations for model-based description of both the software and hardware aspects of a RTES, including the relationships between them. These core concepts can then be used for modeling and performance/schedulability analysis. This is done through annotations applied to existing UML models. 
MARTE shares many commonalities with the following OMG specifications: UML Profile for Modeling Quality of Service and Fault Tolerance Characteristics and Mechanisms, UML Profile for Systems Engineering, Executable UML Foundation, RTCORBA and CCM [MARTE].

The main benefits of MARTE are: providing a common modeling tool for RTES that improves communication among developers, enabling interoperability between development tools in RTES, and fostering the construction of models that may be used to make quantitative predictions regarding real-time and embedded features of systems [MARTE].

MARTE is structured around two main concerns, one to model the features of real-time and embedded systems (MARTE design model) and the other to annotate application models in order to support analysis of system properties [MARTE]. Both concerns reference a shared library, MARTE foundations, that specifies time and concurrency semantics within MARTE models.

In MARTE, it is convenient to group application properties into two categories: functional properties, which are primarily concerned with the purpose of an application (i.e., what it does at run-time); and non-functional properties (NFPs), which are more concerned with its fitness for purpose (i.e., how well it does it or it has to do it) [MARTE]. NFPs use stereotypes to map different properties, such as throughput, delays, overheads, scheduling policies, deadline, or memory usage to UML model elements. They consist of an attribute of one or more instances in terms of a named relationship to a value or values. 


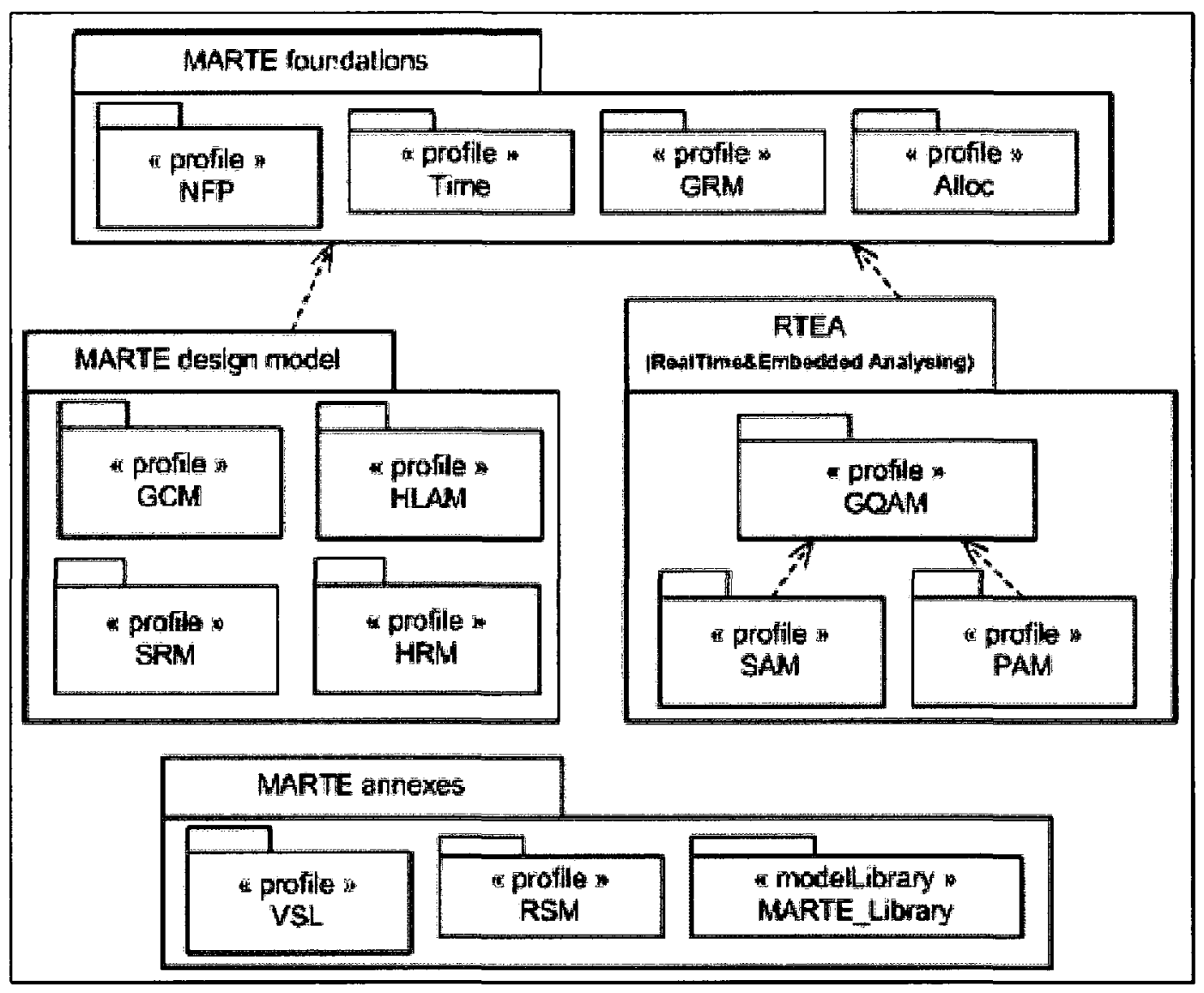

Figure 2-2: MARTE Profile Architecture

[MARTE]

The one profile in MARTE that is of particular relevance to this project is the Performance Analysis Modeling (PAM) profile. Examples of the properties described in the performance analysis domain include mean throughput and delay, or the probability of missing a target response time. PAM stereotypes extend many Generic Quantitative Analysis Modeling (GQAM) profile stereotypes, while adding several domain-specific descriptions. The PAM profile is broken down in to two packages, PAM_Workload and PAM_Resources.

The first package, PAM_Workload, defines stereotypes that describe performance related measures of operations. These include specifications such as operation count, demand or priority. 


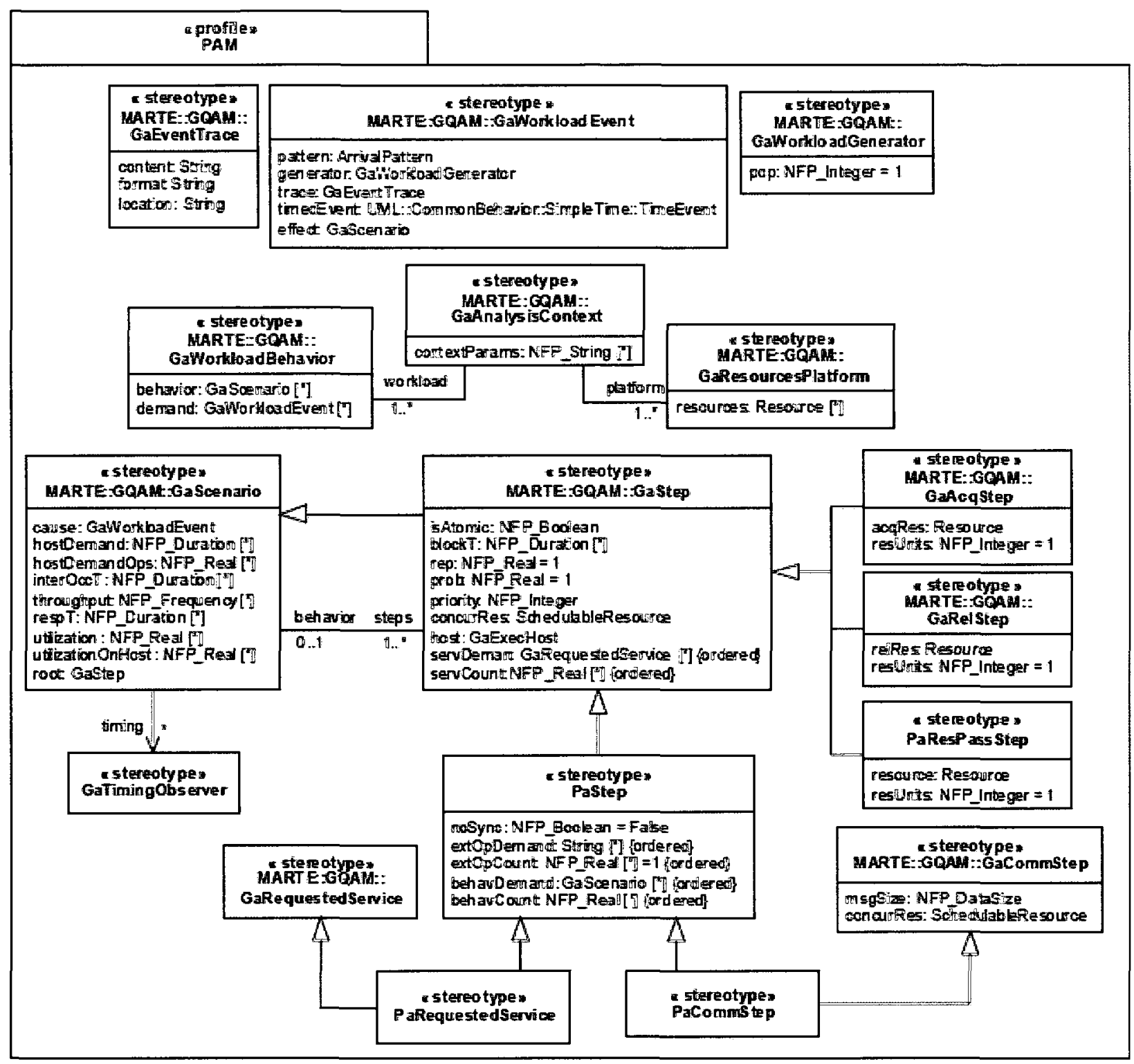

\section{Figure 2-3: PAM_Workload Package [MARTE]}

The second package, PAM_Resources, defines stereotypes for the purpose of detailing how system resources are utilized by the system. This includes properties of resources such as thread pools sizes or throughput. 


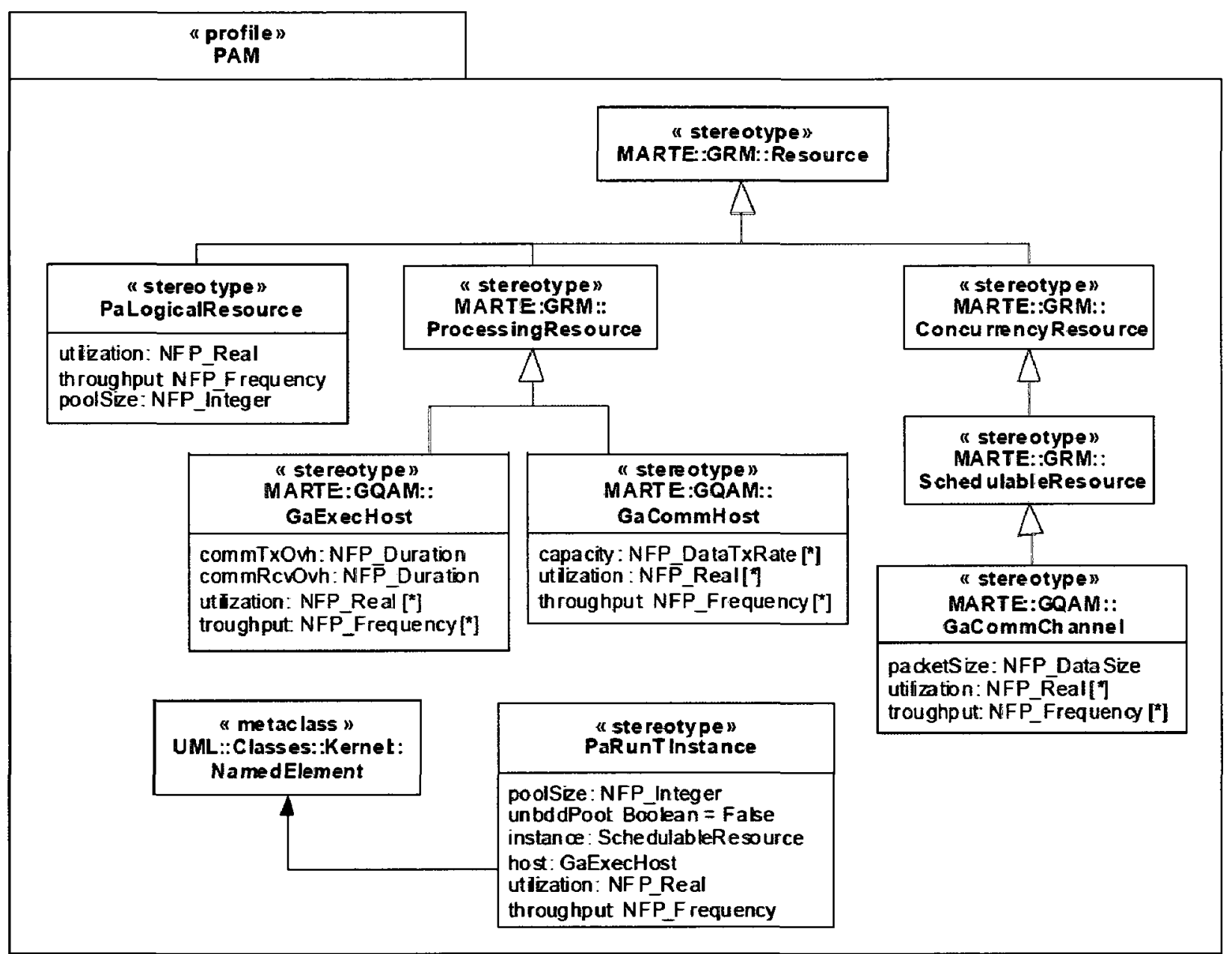

Figure 2-4: PAM_Resources Package

[MARTE]

\subsubsection{SoaML}

SOA systems are networks of participants, whether they are people, organizations, or systems, that provide services for use amongst themselves. It benefits large enterprises that wish to integrate large systems without causing the concerns of the participants to interfere with one another. SoaML provides a metamodel and a UML profile for the specification and design of services within a SOA architecture [SoaML]. It fosters the use of MDA in the design of SOA systems to allow the architecture to be continually implemented even as the technologies of the 
day change. SoaML covers extensions to UML2.1 to support new modeling capabilities such as service identification, service specification, service provider/consumer definition, service policies, and more [SoaML].

\subsubsection{SysML}

Since UML has been explicitly designed to be a general purpose modeling language for software systems, its syntax and semantics have been lacking for systems engineering models. These models require non-software components such as hardware, software, information, processes, personnel, and facilities. These requirements have lead to the development of UML Profile for systems engineering applications. SysML is a domain-specific modeling language that supports the specification, analysis, design, verification and validation of a broad range of complex systems [SysML]. It reuses a subset of the UML 2.1 metamodel, such as diagram components, and provides additional extensions needed for the unique concerns of system models. Some of these concerns include specifying requirements, structure, behaviour, allocations, and constraints on system properties to support engineering analysis [SysML].

\section{$\underline{2.4}$ Modeling Tools and Transformation Languages}

\subsubsection{RSA}

The source models of the transformation process of this study, UML2 Sequence Diagrams, were created with IBM's Rational Software Architect 7.5 [RSA]. RSA is a modeling and development environment that leverages UML methodology for MDD. It is built on the open-source Eclipse IDE [Eclipse]. RSA is widely used around the industry for designing architectures for $\mathrm{C}++$ and Java software applications. 
RSA's main capabilities involve transforming UML models directly in to any of its supported programming languages, which in addition to Java and $\mathrm{C}++$ include: $\mathrm{CH}, \mathrm{XSD}$, CORBA, and several other APIs [RSA]. Existing code can also be reverse-engineered, allowing developers to refine, or integrate existing models in to a new project. RSA also provides advanced visual tools for building UML diagrams.

Since RSA is built on Eclipse, it can make use of the many extensions produced by the platform's community. RSA may also be itself used as a plugin for Eclipse, allowing developers to integrate RSA modeling capabilities in to their coding environments. This was one of the major shortcomings of one of RSA predecessors, Rose RT, where modeling had to be done separately from the development IDEs.

\subsubsection{QVT}

The need for model transformations in MDD has lead to the development of domainspecific transformation languages. In response to a demand for a framework for transformation of MDA-compliant models, OMG released the MOF 2.0 Query/View/Transformations (QVT) standard [QVT]. QVT has 3 domain-specific languages: Relations, Core and Operation Mappings.

The Relations metamodel and language is a declarative specification that supports complex object pattern matching between MOF models, as well as the creation of trace classes to record the details of a transformation execution [QVT]. Relations transformations define a set of conditions of the candidate models that must be satisfied before a transformation from one to the other can take place. Code Fragment 2-1 demonstrates a Relations mapping, along with all the mandatory conditions, for transformations between UML models and RDBMS models [QVT]. 


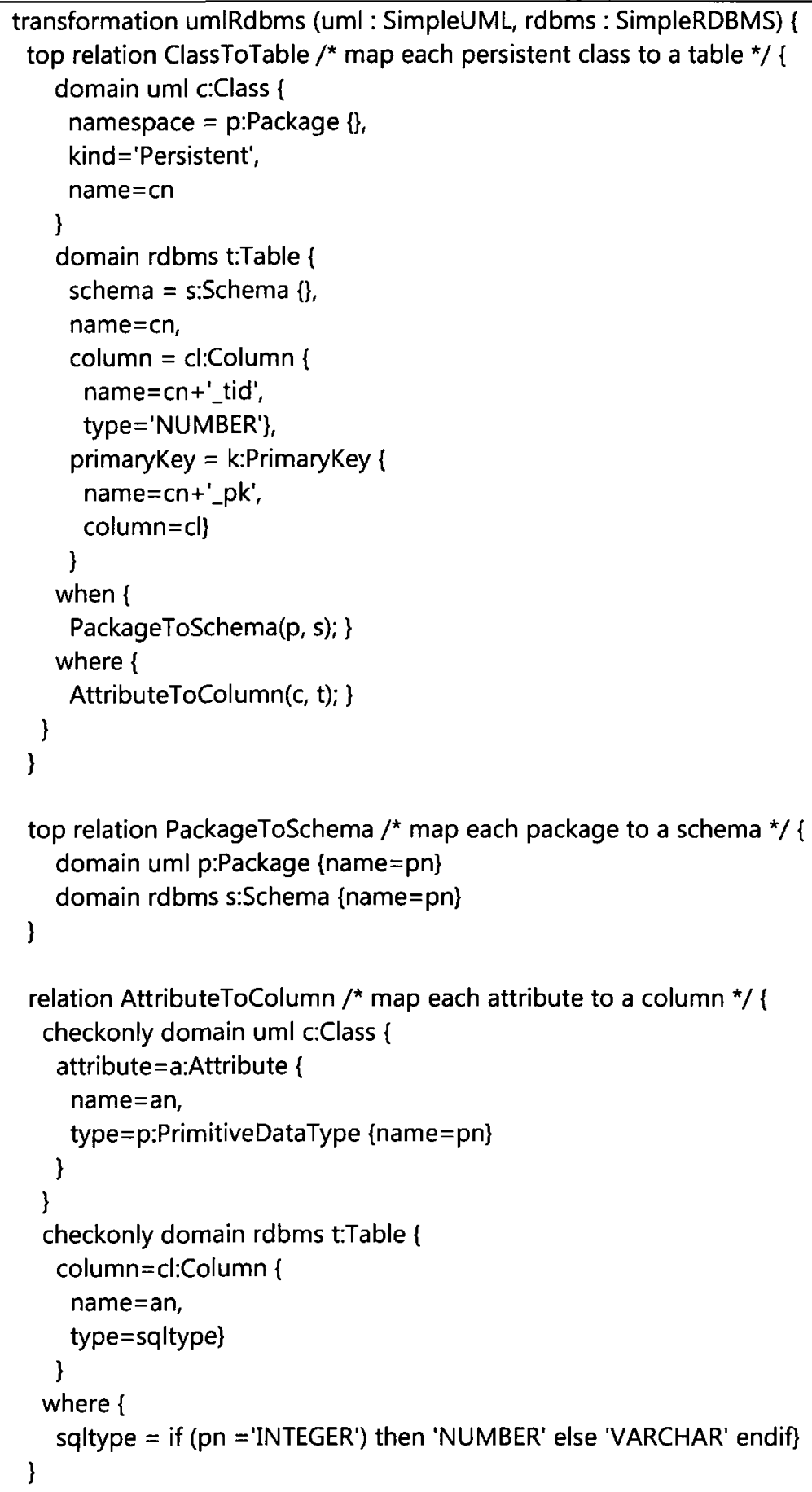

\section{Code Fragment 2-1: QVT Relations Example}

The Core metamodel and language uses minimal extensions to the Essential MOF and OCL languages. It only supports pattern matching over a flat set of variables by evaluating conditions over those variables against a set of models. As a result, transformation descriptions 
are defined in a far more verbose technical manner. In addition, unlike the Relations language, trace models have to be explicitly defined. The core model may be directly implemented, or derived from a Relations model. This is similar to how Java code is transformed in to byte code by the Java compiler [QVT].

The Operation Mappings language is a specification for providing imperative transformations. It supports several extensions to OCL which allow a more procedural syntax for describing complex relations between models. Operations Mappings use the same trace models as Relations language, meaning they can be used interchangeably with the latter.

\subsubsection{ATL}

The Atlas Transformation Language, built by the ATLAS INRIA \& LINA Group, is a model transformation tool that is based on MOF 2.0 QVT Core. Like QVT, ATL is a hybrid between declarative and imperative programming [ATL]. An ATL transformation largely consists of rules written in OCL syntax for converting an element from the source model to element(s) in the target model. These rules allow the transformation to be modularized [Kurtev+07]. These rules may be either of the aforementioned types, matched or imperative.

ATL has two modes of execution, default and refine. Default mode, which was employed in this study, requires the developer to specify the rules that will generate all modified and unmodified elements from the source to target models. Refine mode eases programming in cases where the source and target models are similar.

Matched rules are deterministic and are certified to terminate during execution. Their whole process of execution is also transparent. Imperative rules, which include lazy and called rules, are explicitly specified using complex source-to-target algorithms. They are non- 
deterministic and always have the possibility of not terminating during execution. Both kinds of rules may be used in all cases, however in specific cases design decisions may factor in which type is best used depending on its strengths, weaknesses, and the transformation requirements.

The following is from an example ATL transformation available on the ATL portal [ATL]. Figure 2-5 describes the Metamodels A and B, which the source and target models are based on, respectively.

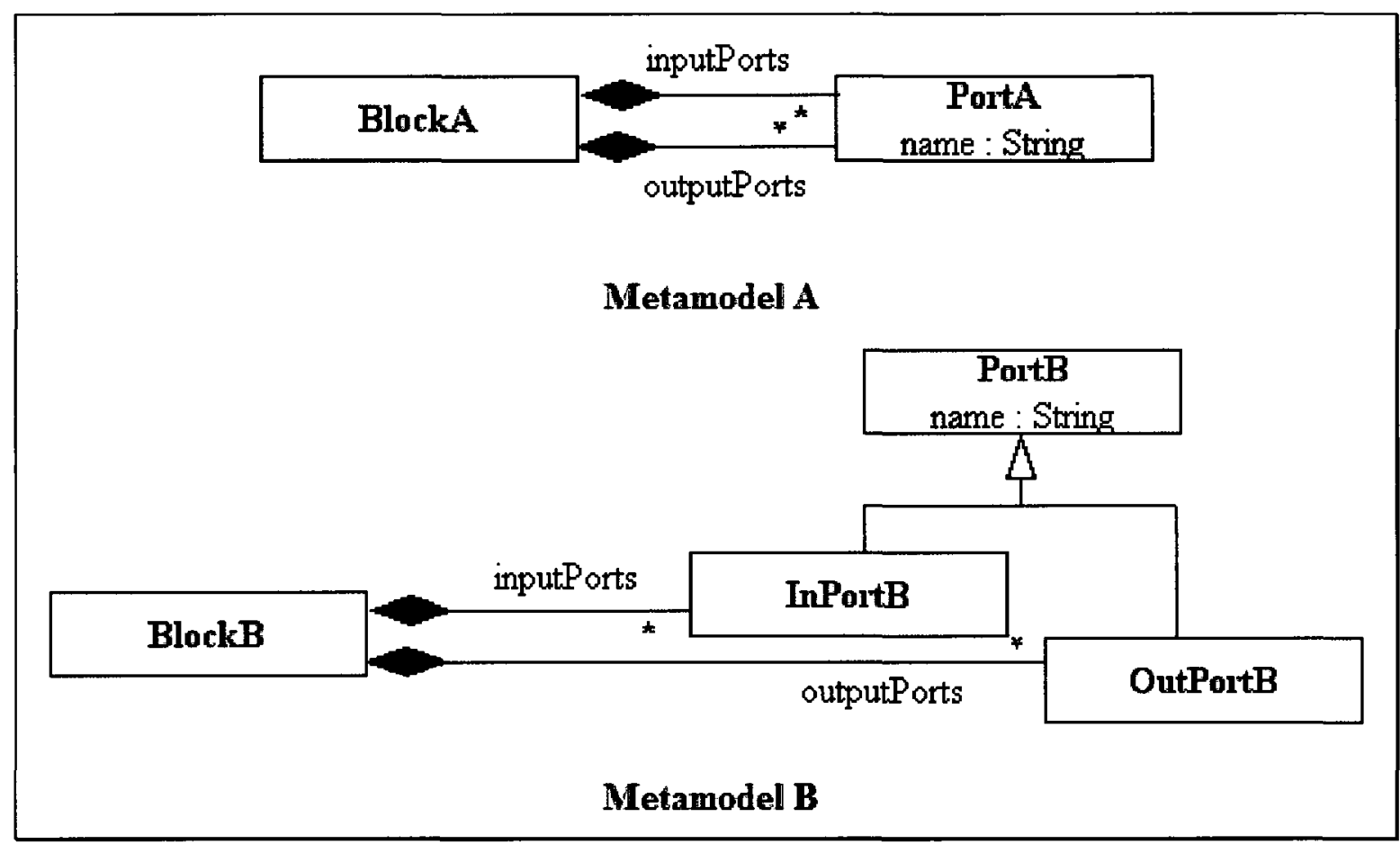

Figure 2-5: ATL Transformation Example - Metamodels [ATL]

The module for this transformation can be written in several different ways, but one common solution is combining the declarative and imperative constructs in ATL as expressed in Code Fragment 2-2. The transformation for BlockA to BlockB is executed automatically through 
the declared matched rule, while lazy rules for transforming PortA in to InPortB and OutPortB are expressed separately so that they me be called imperatively by the matched rule.

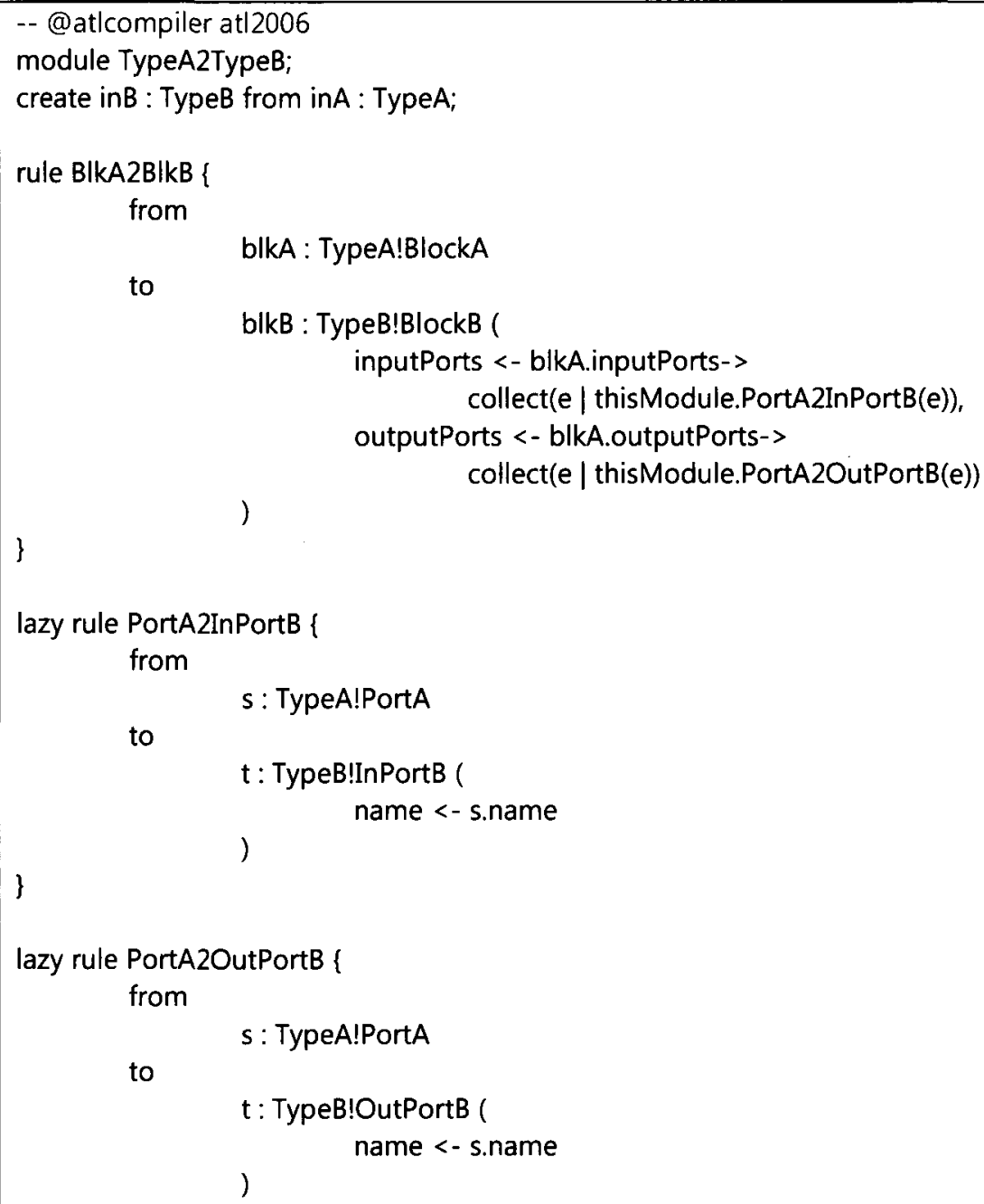

\section{Code Fragment 2-2: ATL Transformation Example - Module} [ATL]

To complement rules, ATL provides helpers, which are analogous to Java methods. They allow the developer to specify factorized ATL code that can be invoked anywhere from within an ATL transformation module [ATL]. All helpers have a name, context type, return value type, code expression, and an optional set of parameters. 
Code Fragment 2-3 is an example of a helper (with no parameters) that can be called by Integer types:

helper context Integer def $:$ double() $:$ Integer $=$ self * 2 ;

\section{Code Fragment 2-3: ATL Helper - Example 1}

[ATL]

Also, it is possible to define a helper that has no context and which can invoked by the ATL module instance (specified by the object "thisModule"):

helper def : $\max (x 1:$ Integer, $x 2:$ Integer $):$ Integer $=\ldots ;$

\section{Code Fragment 2-4: ATL Helper - Example 2}

[ATL]

ATL contains a layered architecture that allows a good degree of flexibility in dealing with other technologies. These layers include:

- ATL programs - the tool (Eclipse or Netbeans) used to write the program

- ATL compiler - transforms programs in to byte code

- ATL VM - executes byte code

- Model Handler Abstraction Layer - translates instructions for model handlers

- Model Handlers - provide programming interfaces for model manipulation (ex. EMF, MDR)

- Model repository - provides storage facilities for models (ex. XMI) 


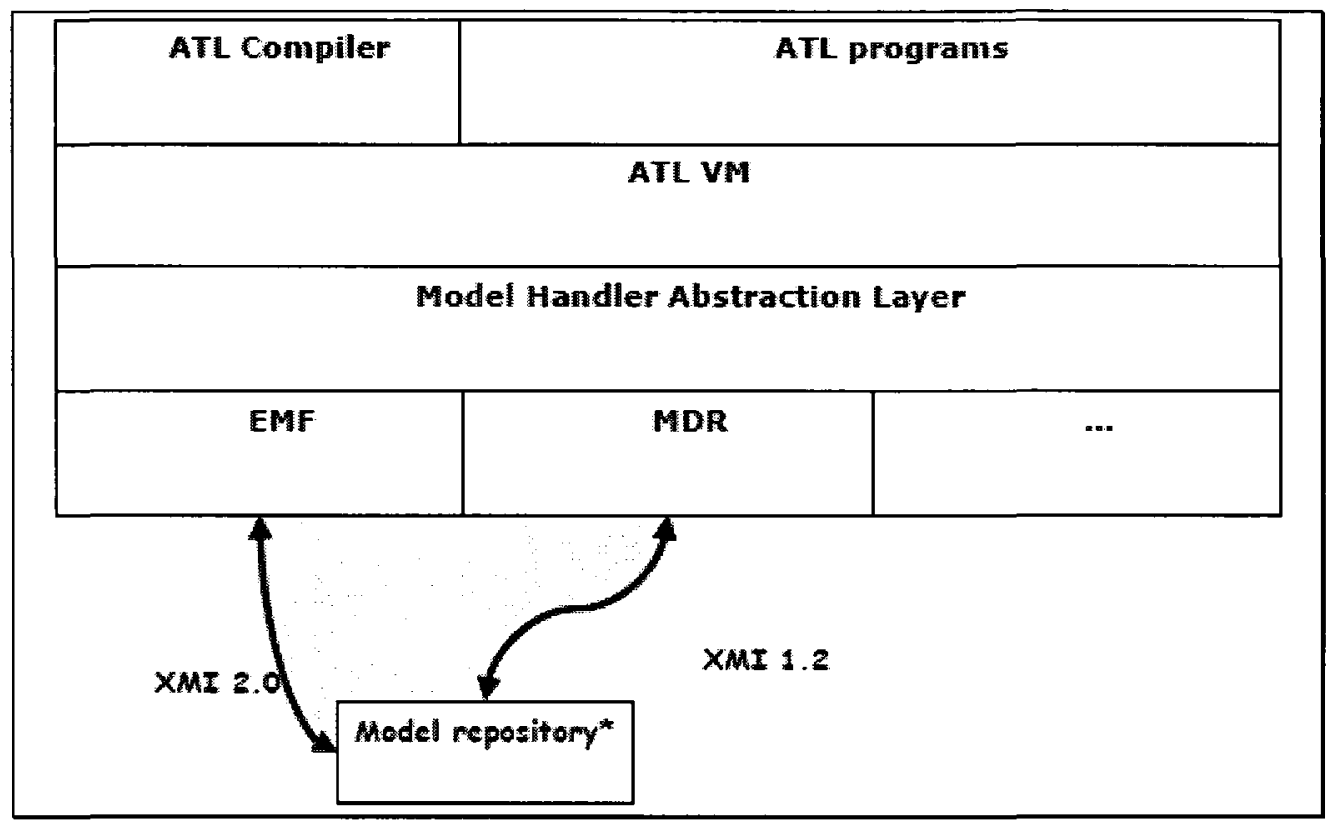

Figure 2-6: ATL Execution Engine

$[\mathrm{JA}+06]$

An ATL transformation is built upon ECORE, an EMF meta-metamodel which defines the very base semantics of ATL itself, the source and target models, and their metamodels.

Figure 2-7 displays the common basic structure of an ATL transformation. Models $M_{a}$ and $M_{b}$ are both based on metamodels $M M_{a}$ and $M M_{b}$ respectively. The transformation model $M_{t}$ in turn is based on $M M_{t}$, the ATL metamodel. Each of the metamodels in the diagram are based on $M M M$, which is the ECORE meta-metamodel.

Since each element in the source model is transformed independently by a matched rule and/or called rules, ATL transformation models have a linear runtime (based on the number of source model elements). This allows a good degree scalability for very large source models. 


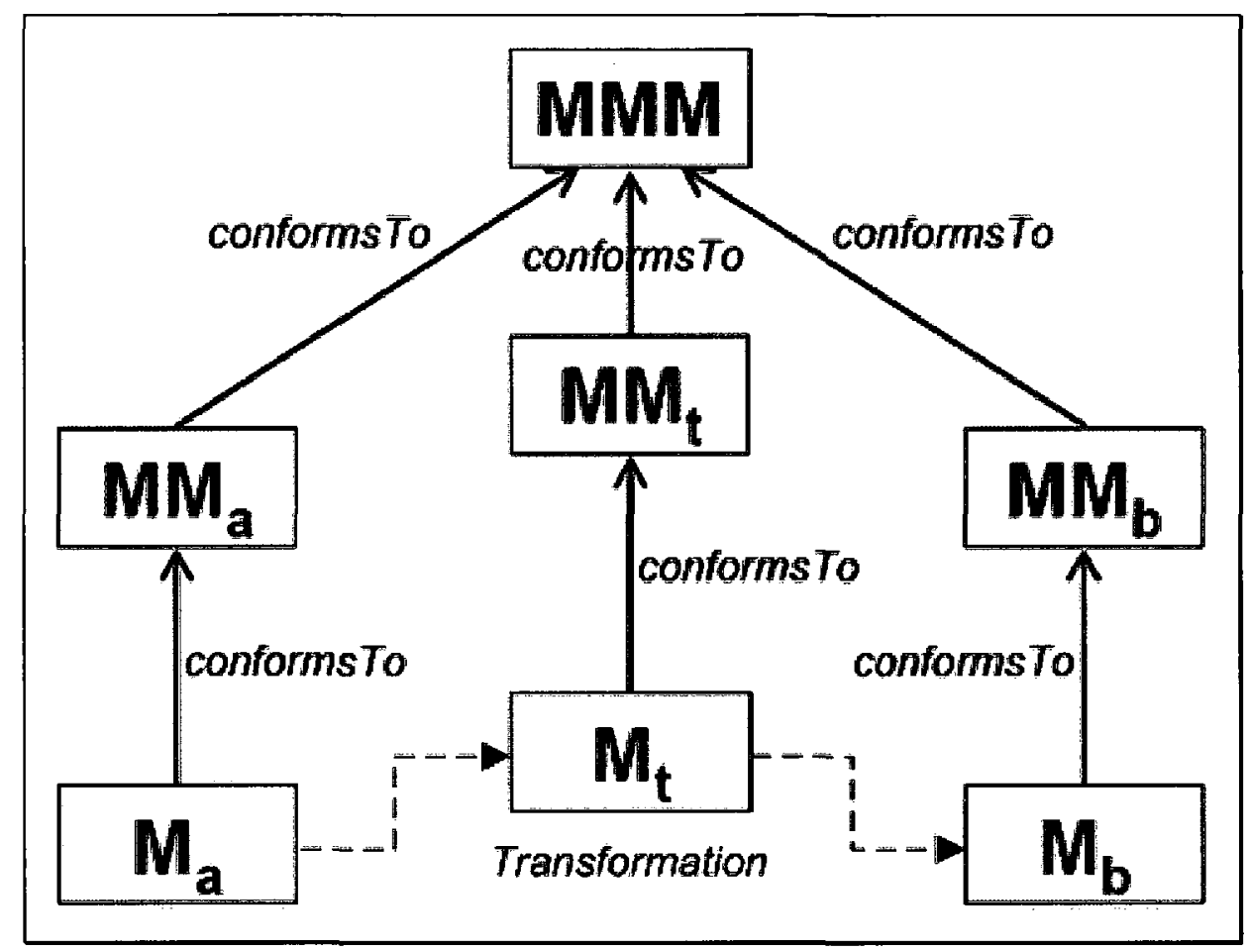

Figure 2-7: ATL Model Structure

[ATL]

\subsection{Methodologies For Model Transformations}

\subsubsection{Overview}

Transformations of software models are complex models themselves, and as such need to be designed, implemented and tested with the same design methodologies that have governed the development of software systems. The area of model transformations is still a relatively young field, but research in to possible design approaches have drawn inspiration from both standard MDE practices and non-conventional techniques. However, model transformation design approaches are often constrained by the multitude of possible paradigms used (such as generalpurpose, graph, or rule-based) and the structure of the source and target metamodels. 


\subsubsection{Transformations By Example}

One paper by Dániel Varró proposes the design and specifications of Model Transformations by Example [Varró+06]. This approach, which is conducted iteratively, involves the semi-automatic derivation of transformation rules from a set of source-target model mappings. After each iteration of the process, the transformation model is either improved or expanded upon. The main advantage of this approach is that the specification (i.e. the prototypical source-target model pairs) and the design (i.e. transformation rules) of a model transformation are kept separate [Varró+06].

The initial step in Model Transformation by Example approach is first assembling a set of prototype mapping models, each being a source and target model pair that captures the relationships between their respective elements.

The second step involves automatically deriving the transformation rules from the prototype mapping models. This also includes rules that derive links between the target elements based on the connectivity of their corresponding source elements.

In the third step, the transformation designer refines the rules by adding attribute conditions or providing generalizations of existing rules [Varró+06]. Attribute conditions refer to situations where there is no direct mapping between source and target elements. In these cases, the transformation designer needs to modify the generated rules manually. As for rule generalizations, in order to reduce the number of model transformation rules, the transformation designer should try to generalize the transformation rules by identifying a more general source pattern from which all the different cases can be derived [Varró+06]. 
Finally, the generated transformation rules are executed on new cases, where the designer can then certify the correctness of the rules. If the resultant target models are unsatisfactory, the new cases can be reintroduced as prototype mapping models in the next iteration of the process.

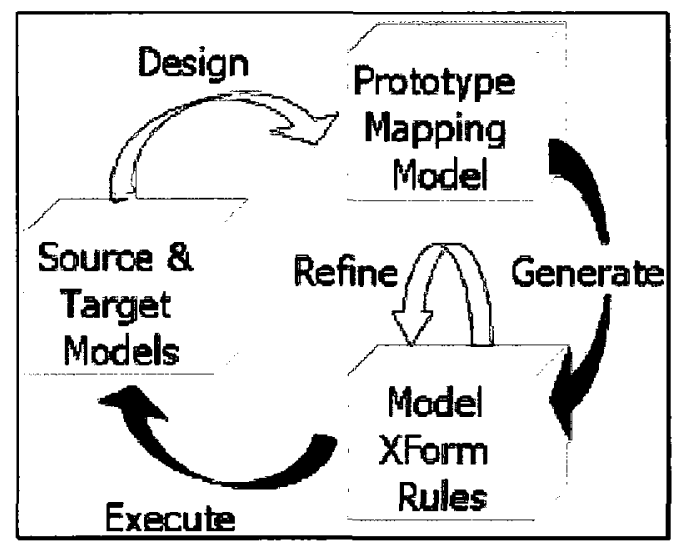

Figure 2-8: Model Transformation by Example Process Overview [Varró+06]

As a proof of concept, the paper performed the process on the transformation of UML class diagrams to a relational database tables. All the steps in the process were performed, with examples of designer interaction illustrated.

Despite the advantage of separating the problem domain from the solutions domain, the Model Transformation by Example approach has some shortcomings. The automatic synthesis of transformation rules only works for generic model mapping prototypes. More complicated mappings with attribute condition require significant refinement from the transformation designer. However despite these limitations, this research has laid the foundations for future work on a fully automated approach to the synthesis of a transformation model. 


\subsubsection{Design Patterns}

Software systems are made up of functional and non-functional requirements, of which the latter are more difficult to implement. Non-functional requirements differ from the functional in that they usually apply to techniques to resolve problems as opposed to the solutions to problems themselves. In the object-oriented domain, NFRs can include concepts such as polymorphism, abstraction, encapsulation, delegation dynamic binding, or inheritance [FHL+06]. What makes NFRs difficult to implement is that their use is subjective. Everyone may have different views on their importance and the potential trade-offs resulting from their use.

Design patterns have long been regarded as a means to capture successful solutions to recurring problems that arise when building software systems [GHJV+95], [FHL+06]. They provide both: a) a way to satisfy NFRs; and b) better solutions to the problem at hand. Using this methodology for transformations, transformation design patterns can be defined as reusable solutions to a general model transformation problem [BM+08]. However, since highly declarative languages, like the QVT standard, have not been around for long, there has not been much research in to model transformation patterns. The research paper, "Reusable Model Transformation Patterns," proposes several design patterns [ISH+08]:

- Mapping Pattern - This pattern establishes one-to-one relationships between source and target model elements in cases where both the source and target models have different metamodels. The predecessor and successor references of each of these elements is mapped to their respective target elements, keeping the ordering of elements in both models relative. This pattern is used in virtually all transformations. 
- Refinement Pattern - This pattern deals strictly with graphs (consisting of nodes connected to each other by edges), whereby the goal is to obtain a more detailed target model by refining an edge to multiple, possible interrelated, edges. In order to add more detail to elements, the Refinement Pattern transforms them in to sub-elements. In contrast to the Mapping Pattern, this pattern deals only with transformation problems where the source and target models use the same metamodel.

- Node Abstraction pattern - Here, nodes are abstracted in the graph source model while keeping all the incidence relations of these nodes. This is useful in transformations where it is only required to remove certain elements from a model, leaving behind only useful elements for a domain-specific analysis. Like the Refinement Pattern, the source and target models must have the same metamodel in order for this pattern to be used.

- Duality pattern - This pattern deals with transformation problems involving models of processes or flow control, where different transformation mappings of nodes or states occur due to their respective relationships to edges or transitions. Put differently, source elements may have "dual characters." Cases like these necessitate more than one transformation mapping for each dual character source element, each determined by the source element's predecessor or successor.

- Flattening pattern - If a model needs to be parsed by a program or analyzed in a specific way, it may need to be flattened. The goal of this pattern is to remove the hierarchy from the source model, where all elements, including their children, reside on one level (while still retaining the correct relationships). It assumes the source and target models have the same metamodel and are both encapsulated by the same root element. 


\section{Chapter 3. Proposed Transformation Approach}

\subsection{Source Model}

The source model of the transformation proposed in the thesis is a UML2 sequence diagram with MARTE performance annotations. A sequence diagram is a scenario notation used to describe the behaviours of distributed systems at an abstract level or to represent a componentbased system's behavioural requirements $[\mathrm{KFJ}+07]$. They describe a finite sequence of operations between different objects.

Sequence diagrams contain model elements such as lifelines, synchronous/ asynchronous messages, alternate/parallel/loop fragments, and behaviour execution specifications. Each of these elements may have an applied stereotype from the MARTE profile, specifying its real-time attributes. The order of appearance of each of these elements in a sequence diagram defines how the resulting target model is structured.

Figure 3-1 represents a simplified view of the UML2 metamodel for sequence diagrams, given for the convenience of the reader. This specific diagram does not contain all the elements from the official OMG specifications. Since the model transformation in this study concerns only a small number of details of the UML2 metamodel, it is more intuitive for the reader to see how those elements are related to one another on a smaller diagram rather than looking at the complete, and complex, UML2 sequence diagram specification. The diagram from Figure 3-1 was constructed by combining relevant parts of several class diagrams from the UML2 Superstructure document [UML2Super]. 


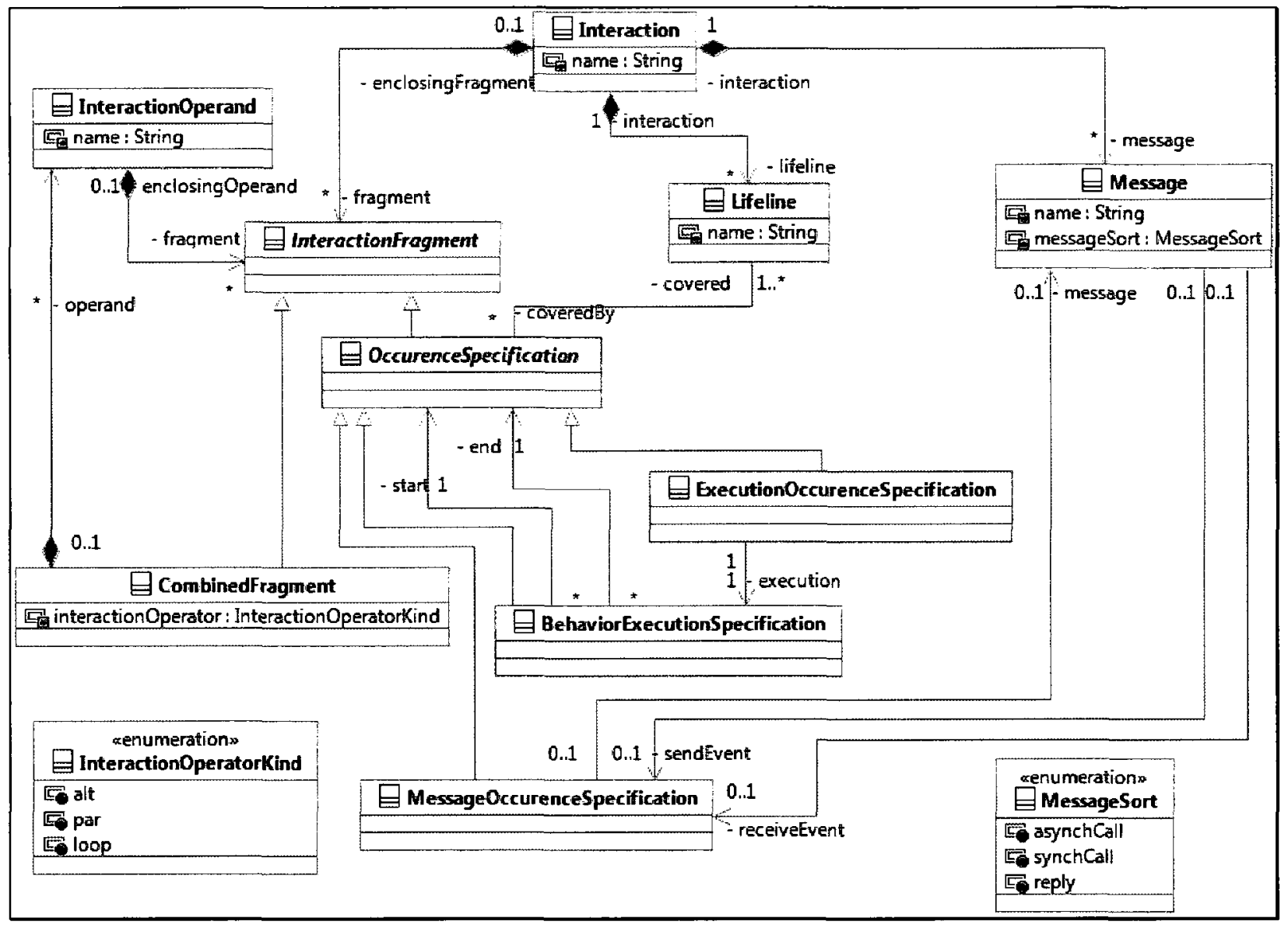

Figure 3-1: Partial UML2 Sequence Diagram Metamodel

Figure 3-2 contains a screenshot of the RSA tool illustrating the graphical notation for a sequence diagram example containing a variety of the different elements within the UML2 metamodel and the MARTE profile. Some of the diagram elements that have an applied MARTE stereotype also have corresponding descriptions of the stereotype attributes. The model elements shown graphically in Figure 3-2 are represented internally by a data structure composed of a set of metaobjects (i.e. instances of the metaclasses of the UML2 metamodel). 


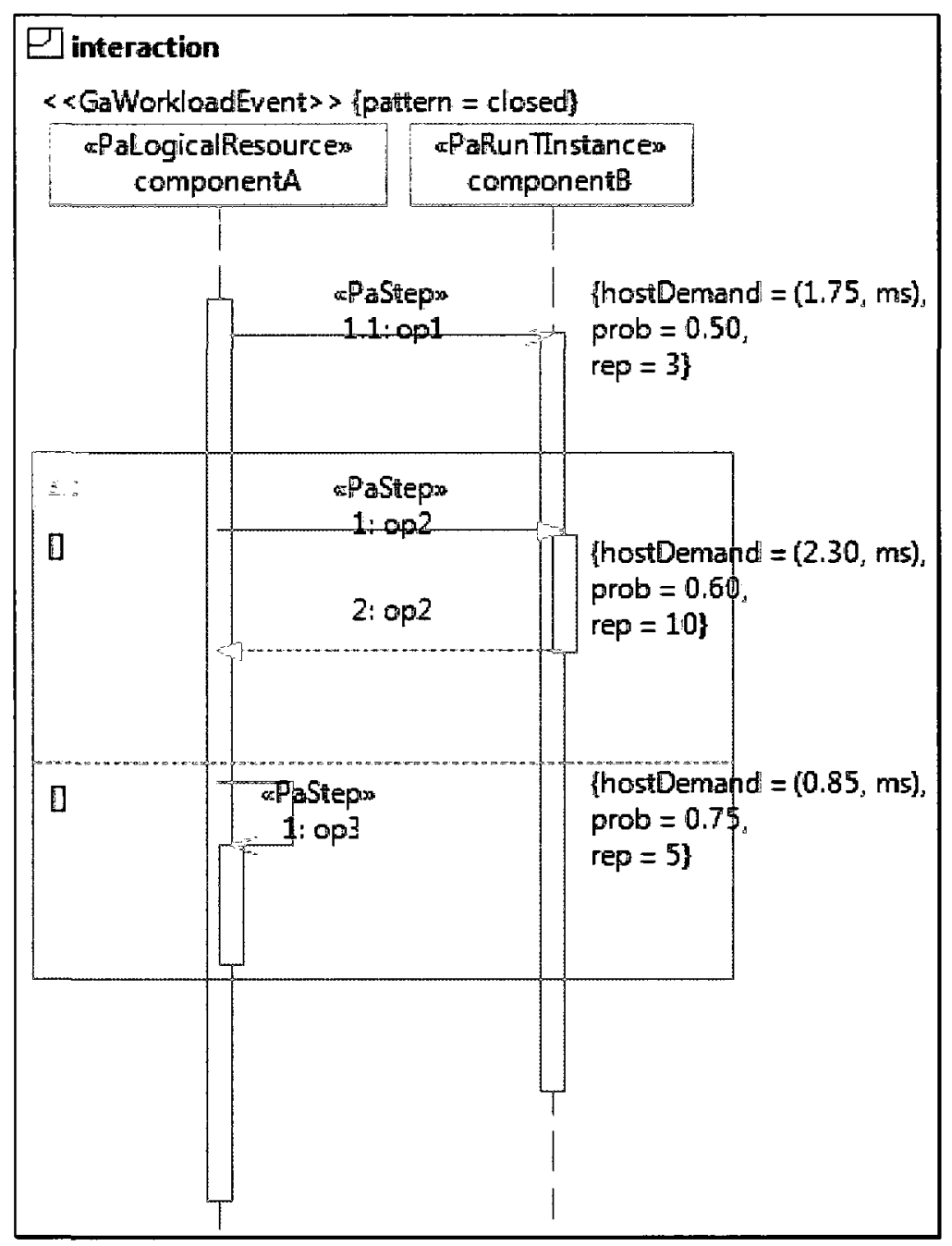

Figure 3-2: Interaction Example

The internal data structure for the Interaction Example given in Figure 3-2 is represented by the UML object diagram in Figure 3-3. The diagram conforms to the UML2 Sequence Diagram metamodel detailed in Figure 3-1, however as noted in Figure 3-3, the ordering of the fragments is intrinsic to the top-down order of appearance of elements within the sequence diagram example from Figure 3-2. 


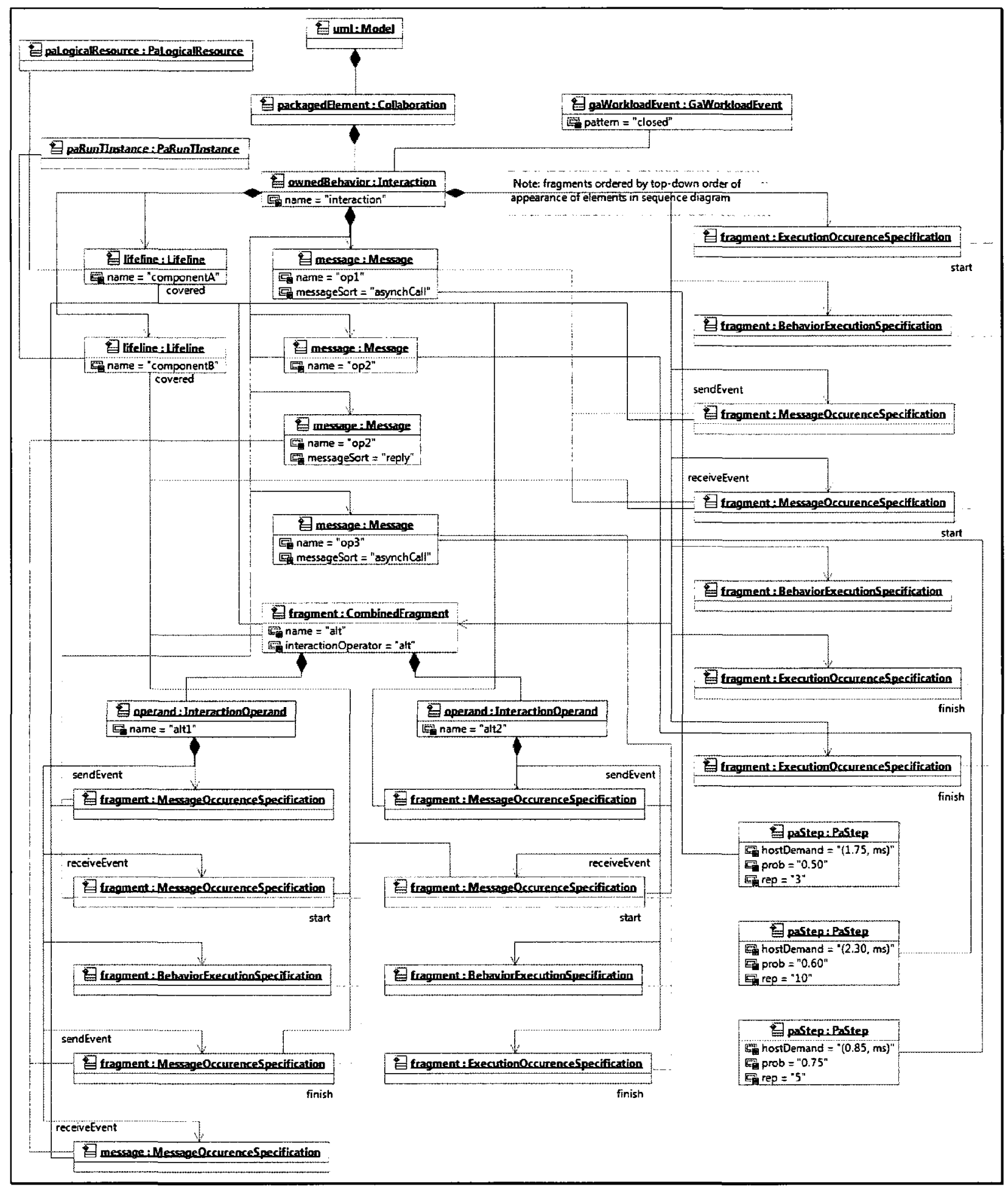

Figure 3-3: Object Diagram showing the Model Elements of the previous Interaction Example 
It should be noted that in this study, we consider that sequence diagrams can have one starting point only; otherwise, the number of transformation rules arising from the numerous element combinations increase considerably. Each message after the first in the model must be preceded by another message, whether being received or sent by the sender lifeline of the message in question. In other words, the proposed transformation can deal only with sequence diagrams containing a single starting point. Similarly, each operand within a fragment must start with a message originating from a common sender lifeline. Unless the fragment is the first event in the sequence diagram, the common sender lifeline must continue from the predecessor (whether it is a message or another fragment).

\subsection{Source to Target Mapping}

At the root of the source model is a UML model element. This model maps to a CSM element, which is the root container of all elements in the target model. Contained within the UML model is an Interaction element, which acts as a container of all elements within the sequence diagram. This element gets transformed in to a Scenario, the container for all elements in a CSM diagram. Generally, every message in a sequence diagram will be transformed in to a Step, along with some other elements depending on the type of message. The only exceptions to this rule are reply messages, which always pair up with synchronous messages.

Table 3-1 illustrates the unconditional mappings between elements from the source and target models:

\begin{tabular}{|l|l|}
\hline \multicolumn{1}{|c|}{ UML+MARTE Sequence Diagram } & \multicolumn{1}{c|}{ CSM } \\
\hline Model & Model \\
\hline Interaction & Scenario \\
\hline
\end{tabular}

Table 3-1: Unconditional Mappings 


\begin{tabular}{|l|l|}
\hline Reflexive Message & Step \\
\hline Synchronous Message & ResourceAcquire, PathConnection, Step \\
\hline Reply Message & Resource Release \\
\hline \multirow{2}{*}{ Asynchronous Message } & ResourceRelease, PathConnection (RR to RA), \\
& ResourceAcquire, PathConnection (RA to Step), \\
\hline Alternate Fragment & Step \\
\hline Parallel Fragment & Branch, Merge \\
\hline Loop Fragment & Fork, Join \\
\hline GaWorkloadEvent & Step, Refinement, Scenario \\
\hline PaLogicalResource & Workload \\
\hline PaRunTInstance & Component (passive) \\
\hline
\end{tabular}

Table 3-1: Unconditional Mappings (cont'd)

In addition to the unconditional mappings there are conditional mappings, where source elements are only transformed in to target elements depending on whether a set of conditions is satisfied. Generally, these conditions are determined by the transformation designer.

\begin{tabular}{|l|l|l|}
\hline \multicolumn{1}{|c|}{$\begin{array}{c}\text { UML+MARTE } \\
\text { Sequence Diagram }\end{array}$} & \multicolumn{1}{|c|}{ Condition } & \multicolumn{1}{|c|}{ CSM } \\
\hline \multirow{4}{*}{ Message (any) } & $\begin{array}{l}\text {-has no preceding messages in sender } \\
\text { lifeline and is not first in any operand }\end{array}$ & $\begin{array}{l}\text { Start, PathConnection (Start to } \\
\text { RA), ResourceAcquire }\end{array}$ \\
\cline { 2 - 3 } & -is first in loop fragment & Start \\
\cline { 2 - 3 } & $\begin{array}{l}\text {-has no succeeding messages in receiver } \\
\text { lifeline and is not last in any operand }\end{array}$ & $\begin{array}{l}\text { PathConnection (Step to RR), } \\
\text { ResourceRelease, } \\
\text { PathConnection (RR to End), } \\
\text { End }\end{array}$ \\
\cline { 2 - 4 } & -is last in loop fragment & $\begin{array}{l}\text { PathConnection (Step to End), } \\
\text { End }\end{array}$ \\
\hline
\end{tabular}

Table 3-2: Conditional Mappings 


\begin{tabular}{|c|c|c|}
\hline \multirow{6}{*}{$\begin{array}{l}\text { Asynchronous } \\
\text { Message }\end{array}$} & $\begin{array}{l}\text {-has no preceding outgoing } \\
\text { asynchronous messages in the sender } \\
\text { lifeline }\end{array}$ & Fork \\
\hline & $\begin{array}{l}\text {-is a fork message (see previous } \\
\text { condition) } \\
\text { - and - } \\
\text {-has no succeeding messages and is not } \\
\text { last in any operand }\end{array}$ & $\begin{array}{l}\text { ResourceRelease, } \\
\text { PathConnection (RR to End), } \\
\text { End }\end{array}$ \\
\hline & $\begin{array}{l}\text {-has no preceding asynchronous } \\
\text { messages in the sender lifeline } \\
\text { - and - } \\
\text {-is last in loop fragment }\end{array}$ & End \\
\hline & $\begin{array}{l}\text {-is joining a behaviour execution thread } \\
\text { that includes a preceding outgoing } \\
\text { asynchronous message } \\
\text { - or - } \\
\text {-is joining a behaviour execution thread } \\
\text { that includes succeeding incoming } \\
\text { asynchronous messages }\end{array}$ & Join \\
\hline & $\begin{array}{l}\text {-is a join message (see previous } \\
\text { condition) } \\
\text { - and - } \\
\text {-preceded by a forking message }\end{array}$ & Step (dummy) \\
\hline & $\begin{array}{l}\text {-is a join message (see previous } \\
\text { condition) } \\
\text { - and - } \\
\text {-succeeded by a forking message }\end{array}$ & Step (dummy) \\
\hline Reflexive Message & $\begin{array}{l}\text {-is not preceded by any forking/joining } \\
\text { messages or alternate/parallel fragments }\end{array}$ & $\begin{array}{l}\text { PathConnection (Predecessor } \\
\text { to Step) }\end{array}$ \\
\hline
\end{tabular}

Table 3-2: Conditional Mappings (cont'd) 


\begin{tabular}{|c|c|c|}
\hline $\begin{array}{l}\text { Synchronous } \\
\text { Message }\end{array}$ & $\begin{array}{l}\text {-is not preceded by any forking/joining } \\
\text { messages or alternate/parallel fragments }\end{array}$ & $\begin{array}{l}\text { PathConnection (Predecessor } \\
\text { to RA) }\end{array}$ \\
\hline Reply Message & $\begin{array}{l}\text {-is not preceded by any forking/joining } \\
\text { messages or alternate/parallel fragments }\end{array}$ & $\begin{array}{l}\text { PathConnection (Predecessor } \\
\text { to RR) }\end{array}$ \\
\hline \multirow{6}{*}{$\begin{array}{l}\text { Alternate/Parallel } \\
\text { Fragment }\end{array}$} & $\begin{array}{l}\text {-is the first occurrence in an } \\
\text { alternate/parallel fragment } \\
\text { - or - } \\
\text {-is preceded by another alternate/parallel } \\
\text { fragment } \\
\text { - or - } \\
\text {-is preceded by a forking/joining } \\
\text { message }\end{array}$ & Step (dummy) \\
\hline & $\begin{array}{l}\text {-is the last occurrence in an } \\
\text { alternate/parallel fragment } \\
\text { - or - } \\
\text {-is succeeded by a forking/joining } \\
\text { message }\end{array}$ & Step (dummy) \\
\hline & -is the first occurrence in a loop fragment & Start \\
\hline & $\begin{array}{l}\text {-has no preceding messages and is not } \\
\text { first in any operand }\end{array}$ & $\begin{array}{l}\text { Start, PathConnection (Start to } \\
\text { RA), ResourceAcquire }\end{array}$ \\
\hline & $\begin{array}{l}\text {-has no succeeding messages and is not } \\
\text { last in any operand }\end{array}$ & $\begin{array}{l}\text { ResourceRelease, } \\
\text { PathConnection (RR to End), } \\
\text { End }\end{array}$ \\
\hline & -is the last occurrence in a loop fragment & End \\
\hline \multirow{2}{*}{$\begin{array}{l}\text { Alternate/Parallel } \\
\text { Operand }\end{array}$} & -begins with a forking message & Step (dummy) \\
\hline & -ends with a forking/joining message & Step (dummy) \\
\hline Loop Fragment & $\begin{array}{l}\text {-is not preceded by any forking/joining } \\
\text { messages or alternate/parallel fragments }\end{array}$ & $\begin{array}{l}\text { PathConnection (Predecessor } \\
\text { to Step) }\end{array}$ \\
\hline
\end{tabular}

Table 3-2: Conditional Mappings (cont'd) 


\begin{tabular}{|l|l|l|}
\hline Loop Fragment & $\begin{array}{l}\text {-is last occurrence in a loop fragment } \\
- \text { or - } \\
\text {-has no succeeding message }\end{array}$ & PathConnection (Step to RR) \\
\hline
\end{tabular}

Table 3-2: Conditional Mappings (cont'd)

Elements in the target model are linked to each other based on how their source model counterparts are linked to each other. In the case of elements generated from conditional mappings, they are linked to the target model counterpart of the source element that lead to the conditional mapping. Also, depending on where they are located in the target model, conditionally mapped elements may also be linked to the target model counterparts of the source element's predecessor or successor (ex. join message succeeded by asynchronous messages).

\section{$\underline{\text { 3.3 Transformation Rules }}$}

\subsubsection{Overview}

In this section, the main transformation rules, corresponding to the source-to-target mappings from the previous section, are presented. Although the transformation rules are implemented in ATL, which is a textual language, we decided to present them in this section using the graphical notation of the QVT relations language because it makes each transformation more intuitive and easier to read. In the QVT transformation diagram syntax, a symbol (pictured in the center of Figure 3-4) is introduced, which represents the transformation between two domains: the source metamodel (UML+MARTE) on the left and the target package (CSM) on the right. Additionally, QVT transformation diagrams show the mappings of attributes in the source model and how they correspond to attributes in the target model (ex. model.name in Figure 3-4). 


\subsubsection{UML Model}

A UML Model is the root container of a source model. Each UML Model is transformed in to a CSM Model, the root container of the target model. CSM Models encapsulate all elements related to a CSM including scenarios, refinements, workloads, and resources.

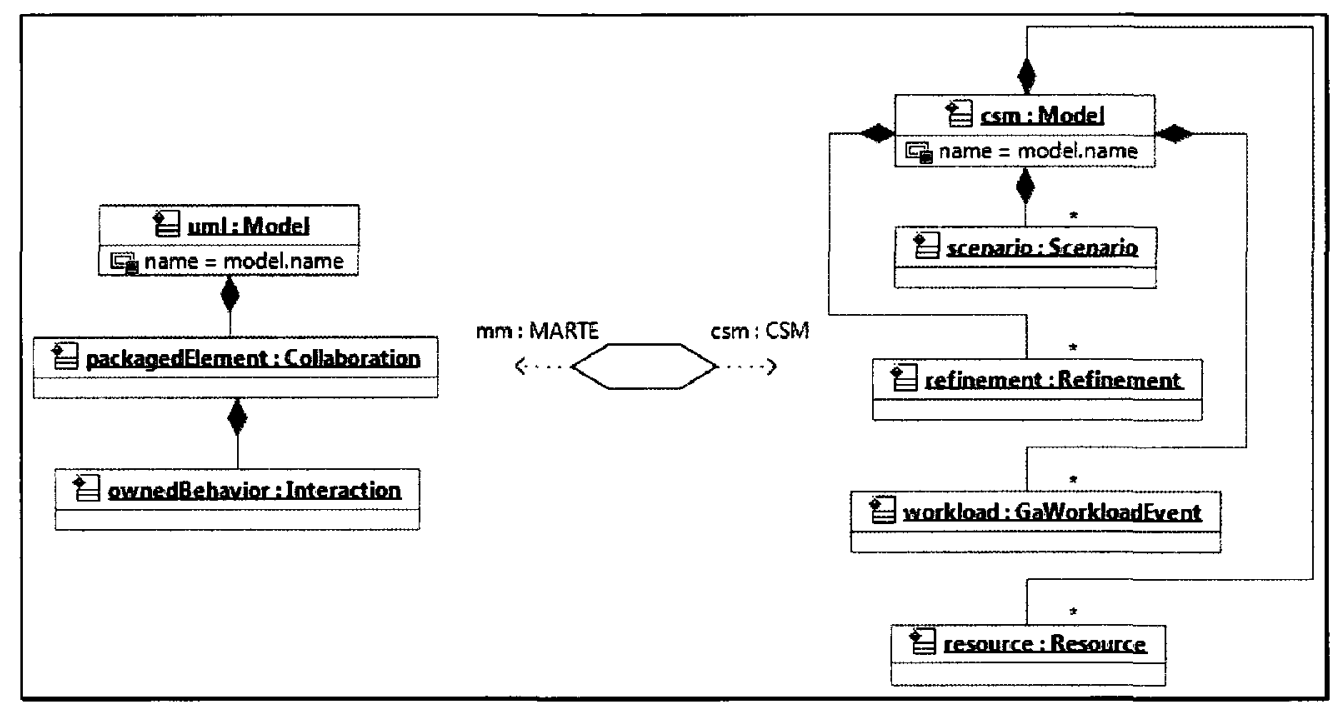

Figure 3-4: UML Model - Transformation Rule

\subsubsection{Interaction}

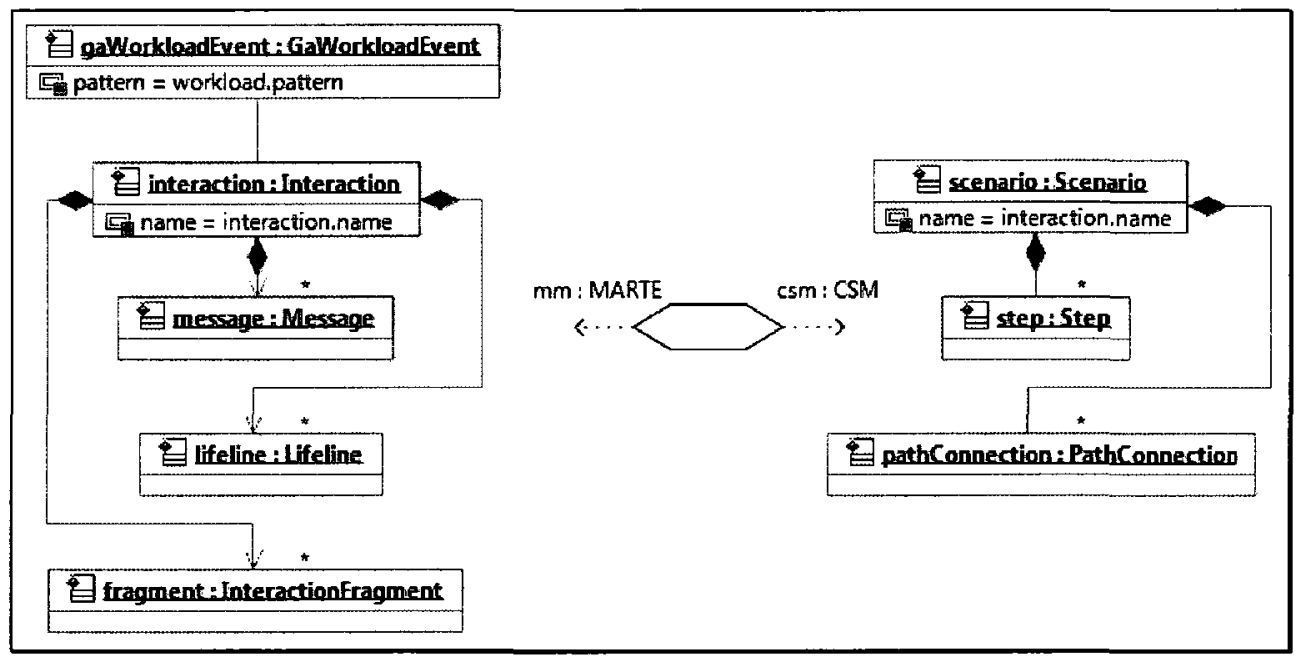

Figure 3-5: Interaction - Transformation Rule 
An Interaction contains all the visual elements that make up a UML2 Sequence Diagram.

Each Interaction maps to a Scenario in the target model. Scenarios are comprised of collections of Steps and PathConnections that make up a CSM.

\subsubsection{Reflexive Message}

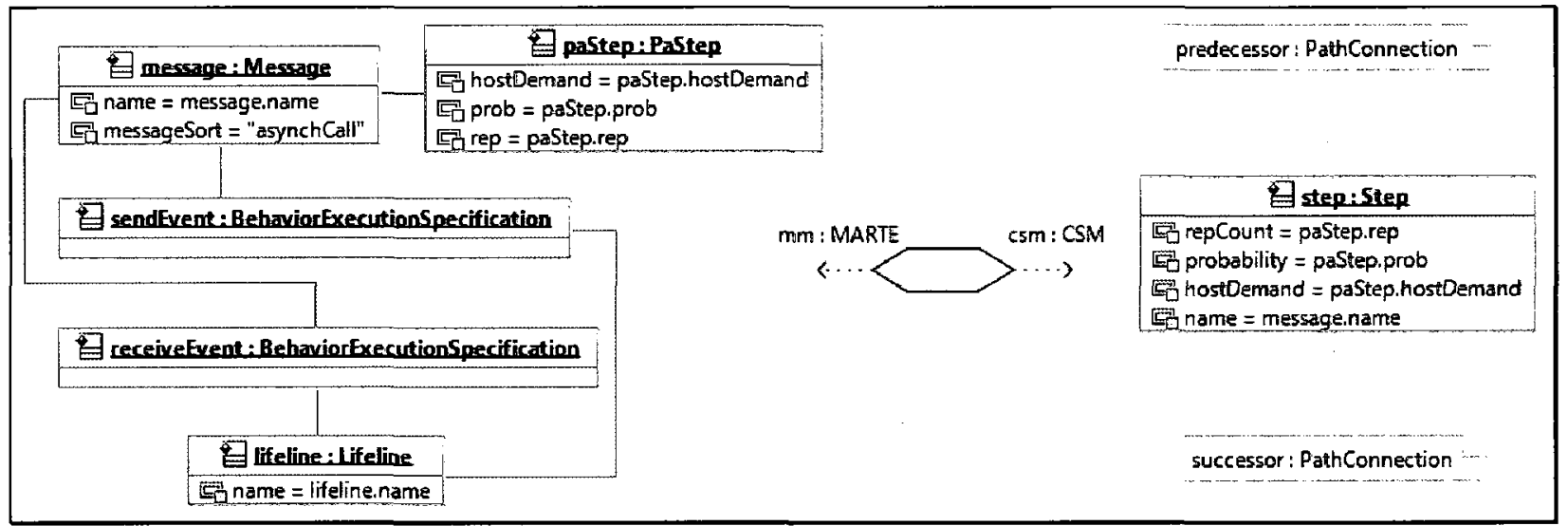

Figure 3-6: Reflexive Message - Transformation Rule

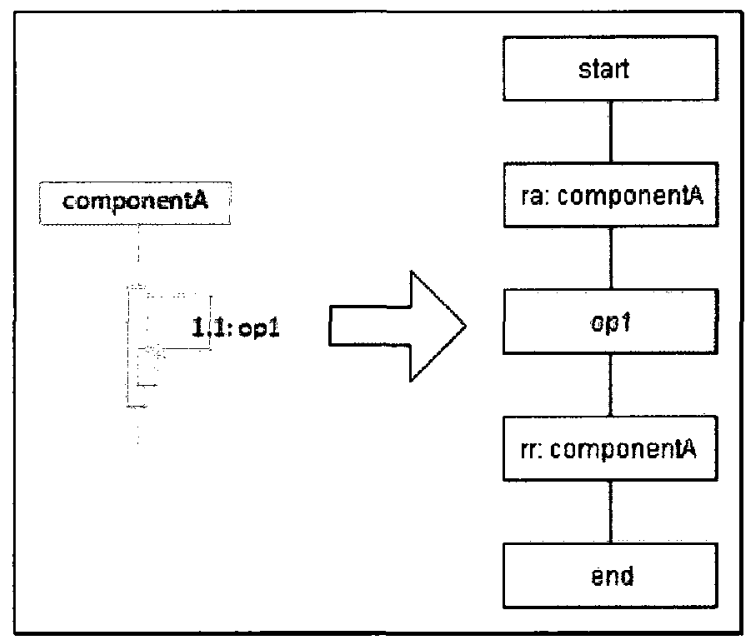

Figure 3-7: Reflexive Message - Transformation Example 
The transformation of reflexive messages is relatively simple. The message is transformed in to a single Step connected to its predecessor and successor. Resources don't need to be acquired or released unless the message in the target model has no preceding or successor messages, respectively. Target elements that are generated from other transformation mappings (like predecessors and successors) are visualized by a yellow note (in Figure 3-6). These same elements are not highlighted in the instance diagrams (like the one in Figure 3-7), whereas the conditionally mapped elements (in this case "op1") are shaded in gray. Note that "ra" and "rr" stand for Resource Acquire and Resource Release, respectively.

\subsubsection{Synchronous Message}

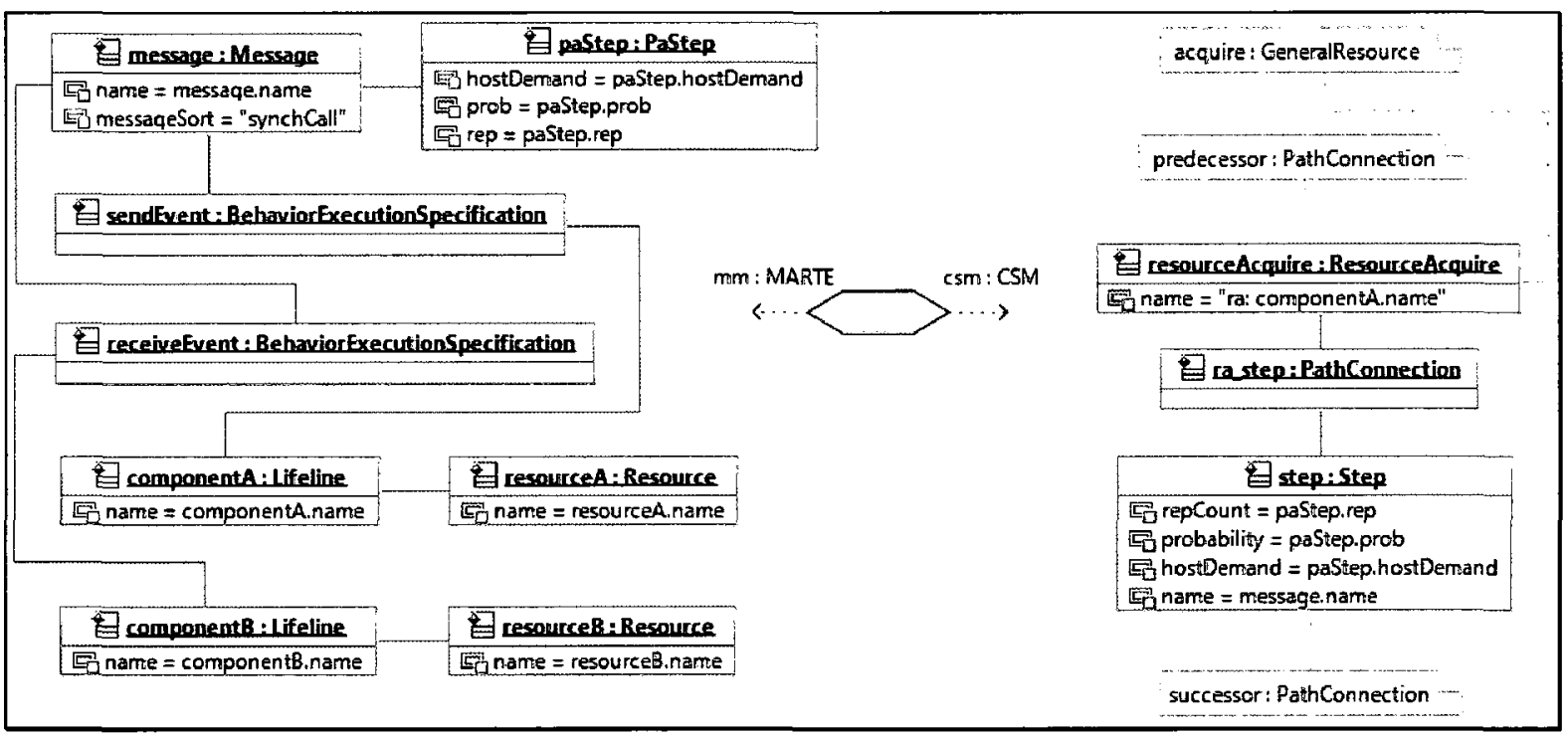

Figure 3-8: Synchronous Message - Transformation Rule 


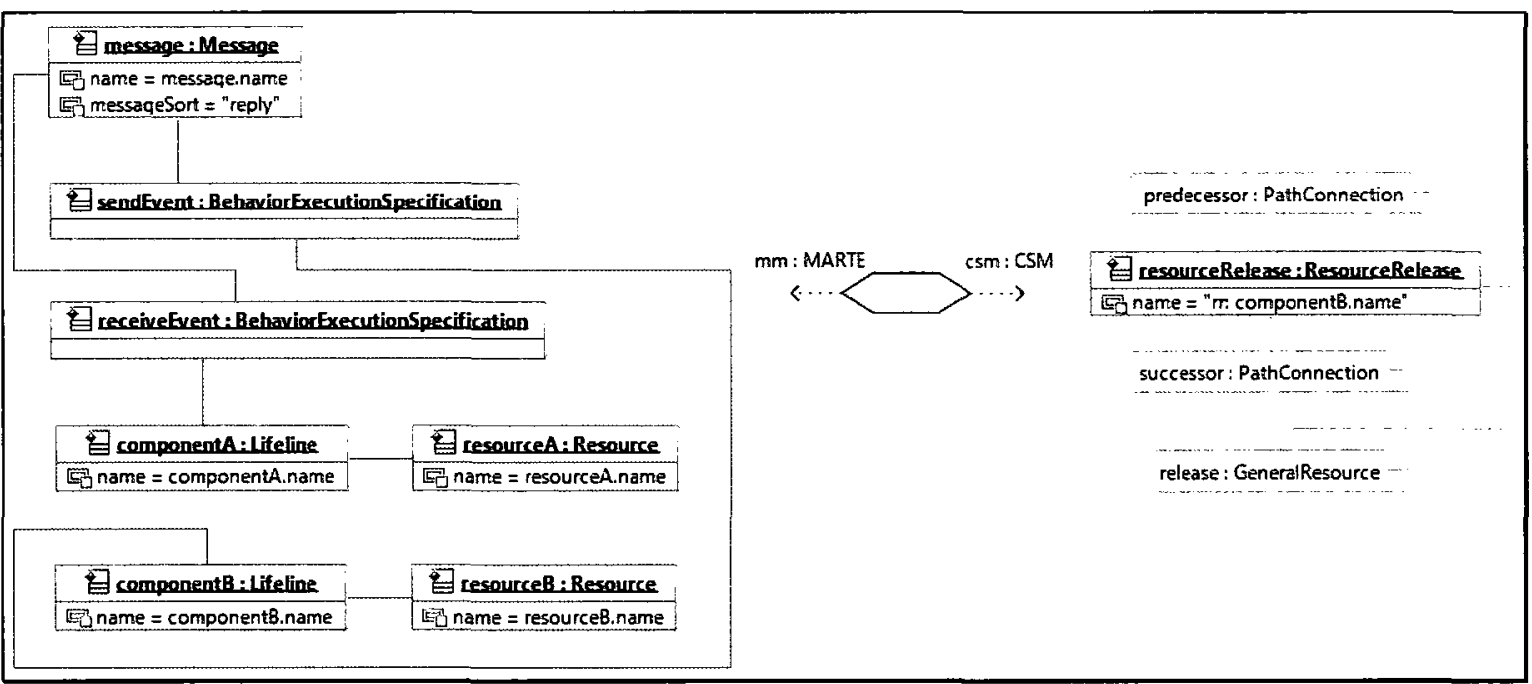

Figure 3-9: Reply Message - Transformation Rule

Synchronous/Reply message pairs are transformed in to three elements. First, the synchronous message is transformed in to a ResourceAcquire of the receiver lifeline, a PathConnection, and a Step of the operation. Then whatever succeeds the synchronous message determines what successor to the Step is in the target model. In this case, only the Reply succeeds the Synchronous Message, therefore a ResourceRelease follows the Step.

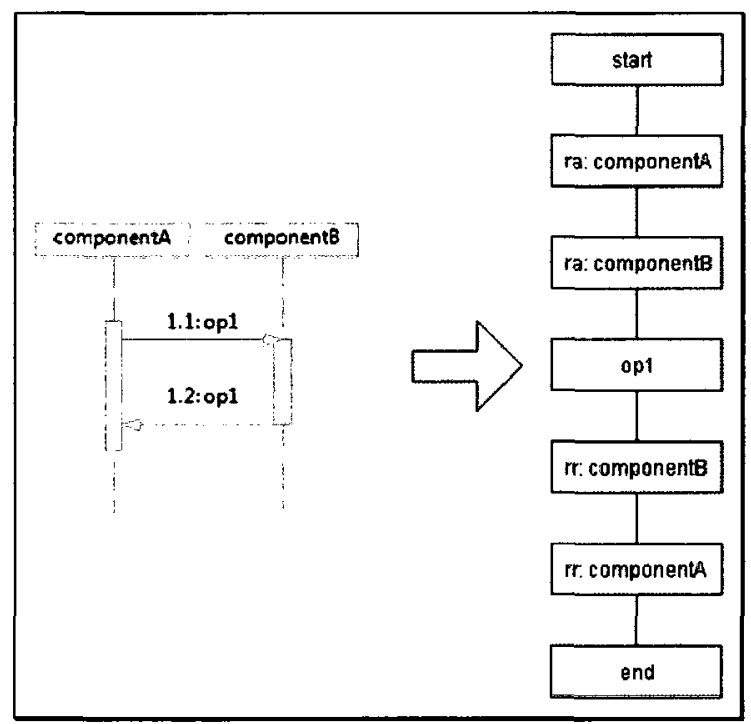

Figure 3-10: Synchronous/Reply Messages - Transformation Example 


\subsubsection{Asynchronous Message}

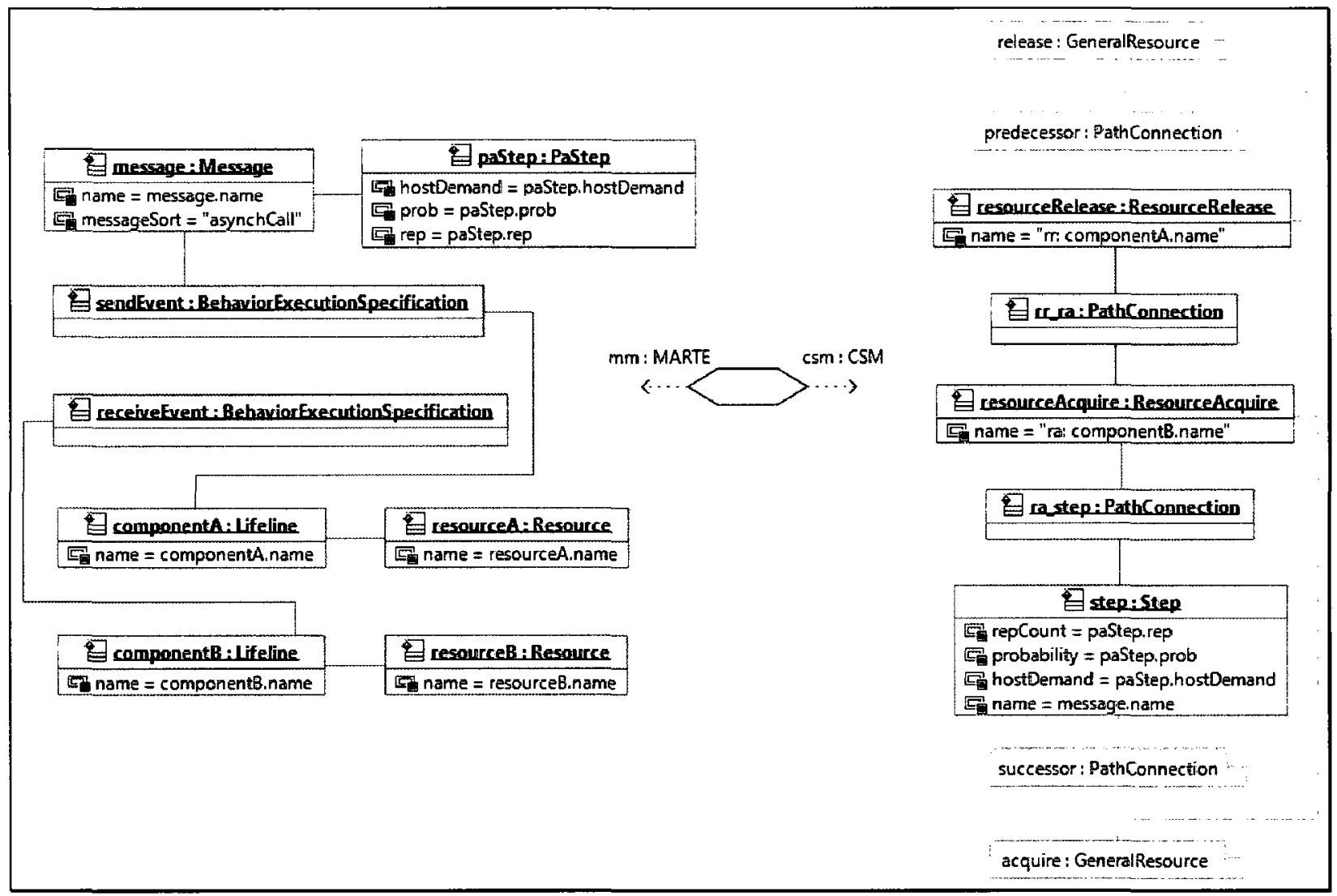

Figure 3-11: Asynchronous Message - Transformation Rule

Asynchronous messages have more complex transformation logic then the previous examples. If an asynchronous message is the first of a group (it has no preceding asynchronous messages with the same source lifeline), then a Fork PathConnection is generated. The Fork is connected to all the transformed elements of the messages succeeding within the asynchronous message group. The asynchronous message group ends where the first non-asynchronous message, or the end of the lifeline, is encountered.

The five elements an asynchronous message is transformed in to are: a ResourceRelease of the source lifeline, a PathConnection connecting it to the next element, a ResourceAcquire of 
the target lifeline, another PathConnection connecting it to the next element, and a Step of the operation. In the example of Figure 3-12, an end of the lifeline is encountered by the asynchronous message group, in which case the fork is connected to the ResourceRelease of the sender lifeline of the message.

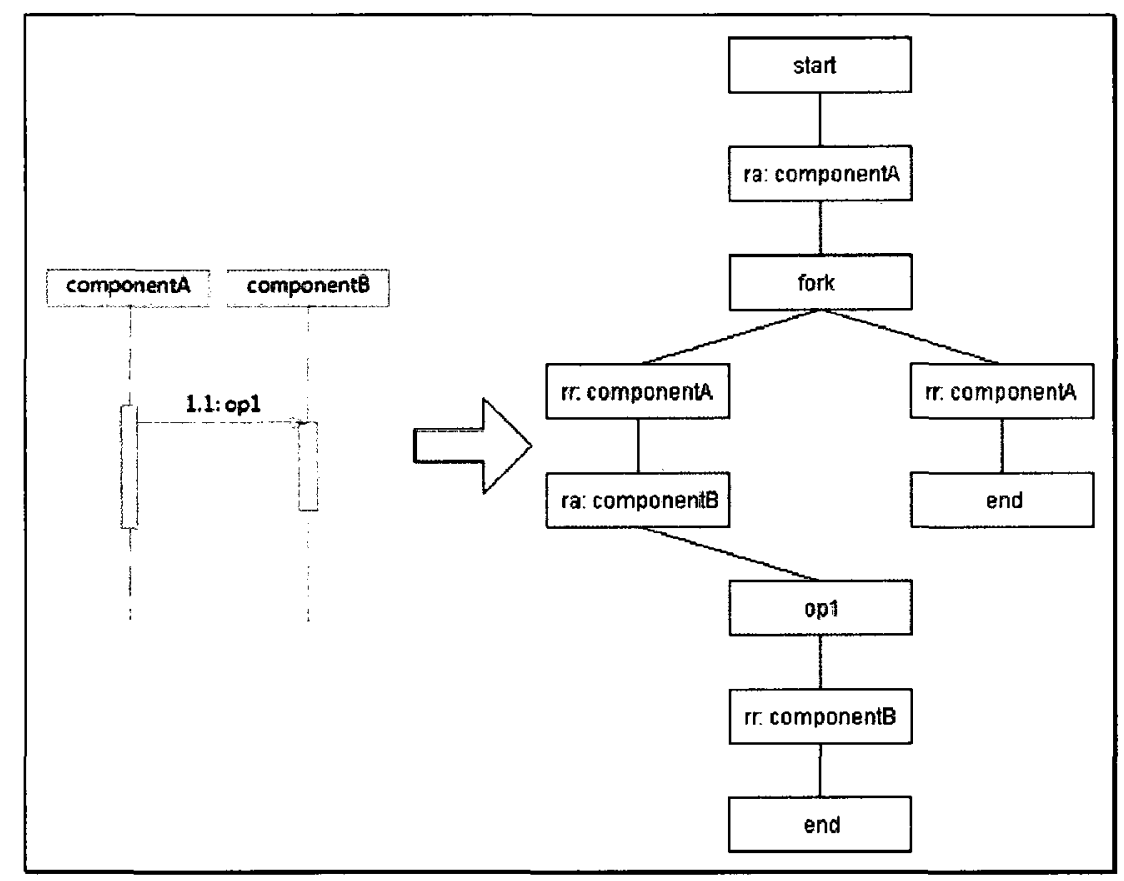

Figure 3-12: Asynchronous Message - Transformation Example

\subsubsection{Alternate Fragment}

Alternate fragments represent decision points in the sequence diagram, and so Branch and Merge PathConnections are generated from the transformation. Each operand from the fragment that contains messages has its transformed elements connected to the Branch and Merge. In cases where another PathConnection (such as the Fork from an asynchronous message) appears after the Branch or before the Merge, a dummy Step is inserted to fill in the gap. 


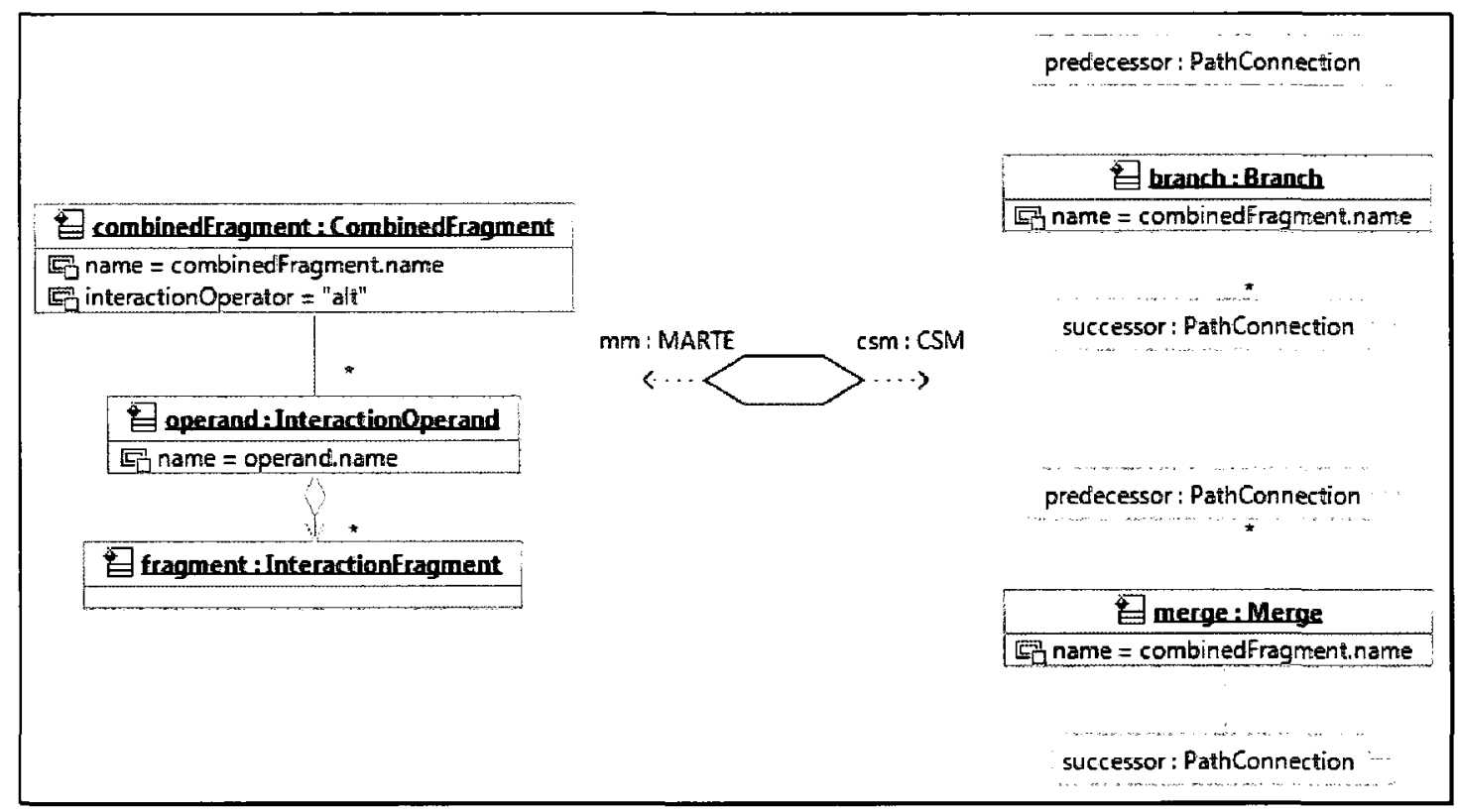

Figure 3-13: Alternate Fragment - Transformation Rule

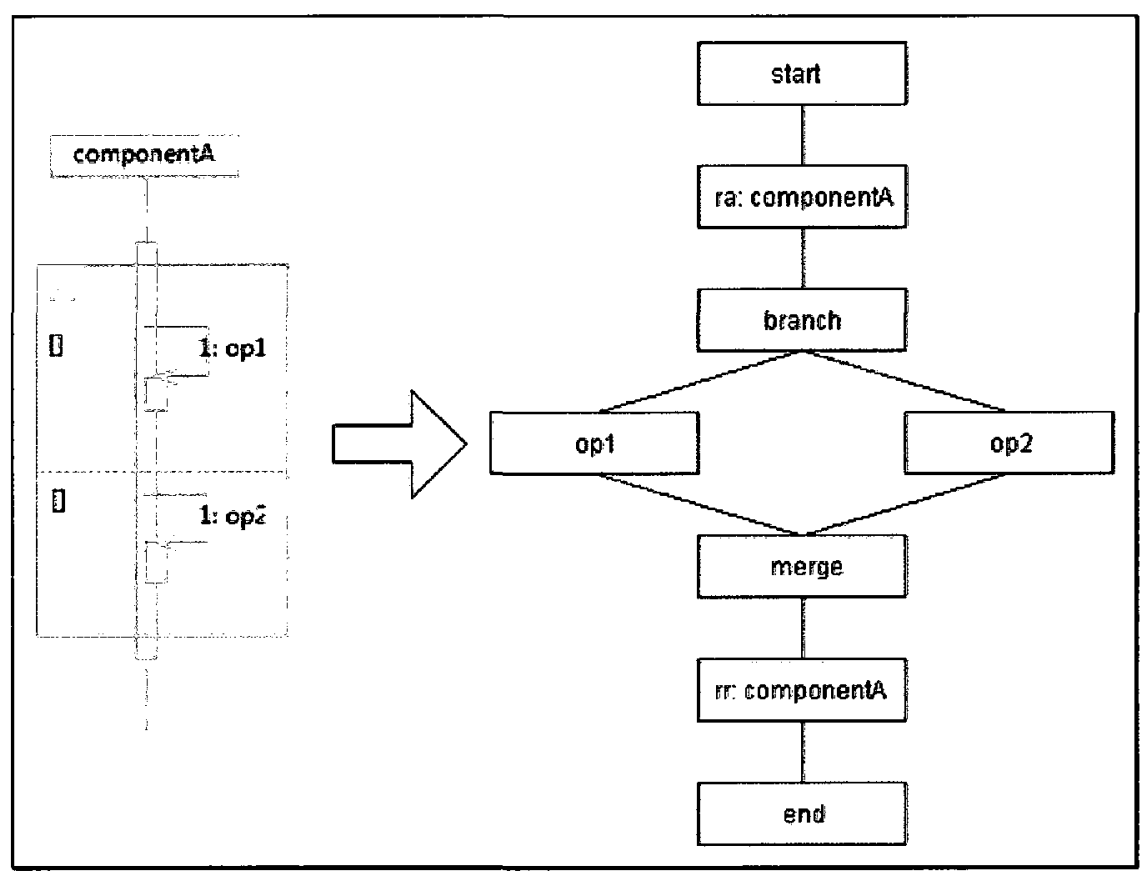

Figure 3-14: Alternative Fragment - Transformation Example 


\subsubsection{Parallel Fragment}

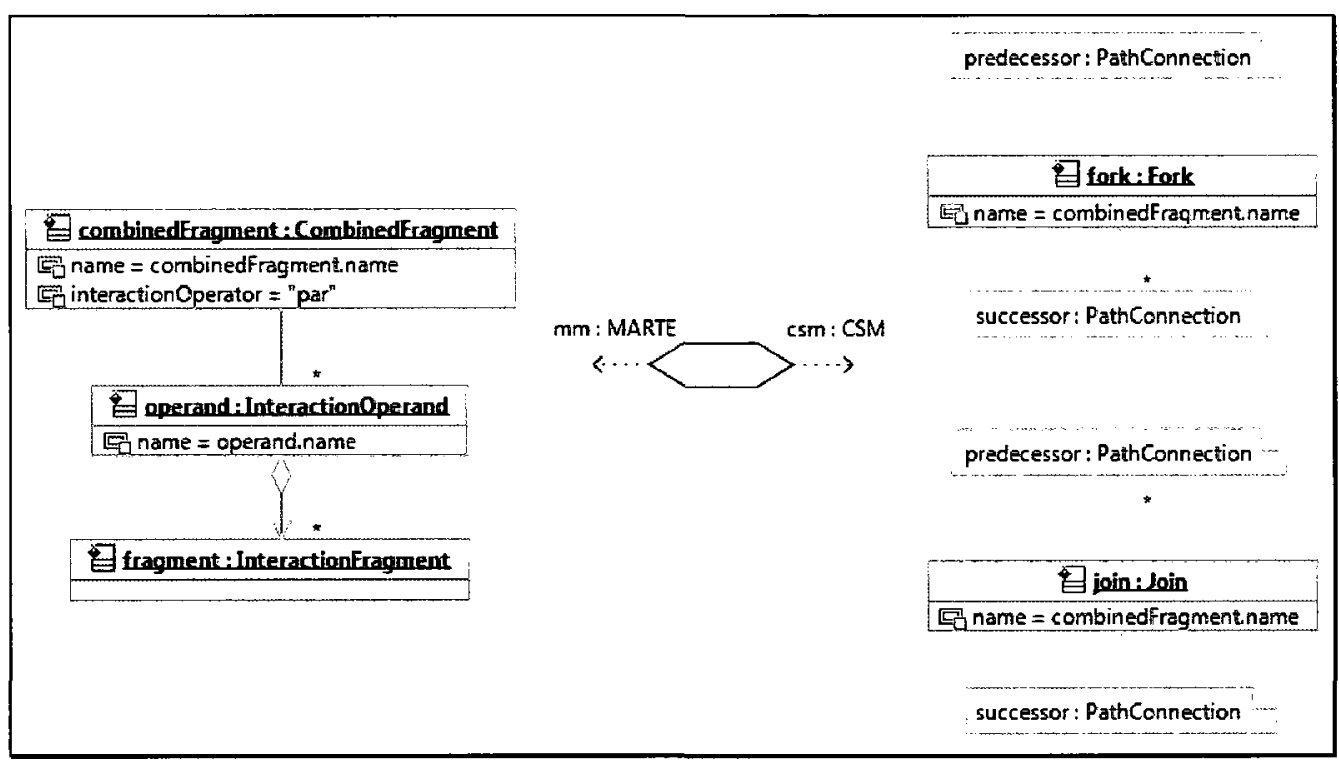

Figure 3-15: Parallel Fragment - Transformation Rule

Parallel fragments are similar to Alternate fragments, although the key difference is each operand is run as an independent concurrent process. Instead of generating Branches and Merges, Forks and Joins are transformed from Parallel fragments.

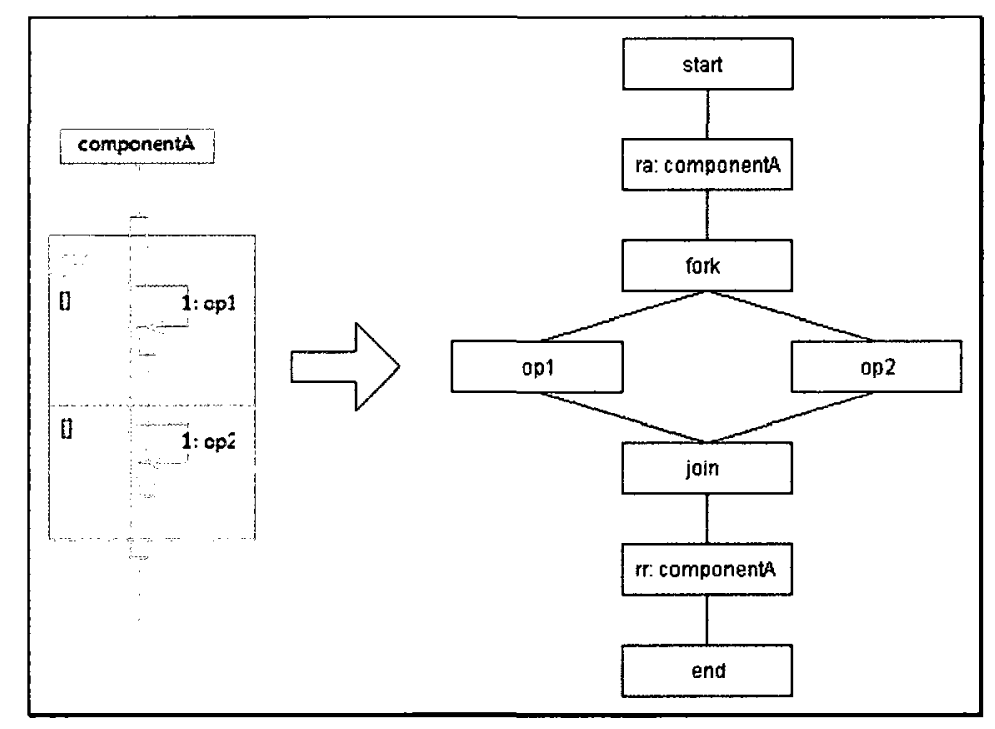

Figure 3-16: Parallel Fragment - Transformation Example 


\subsubsection{Loop Fragment}

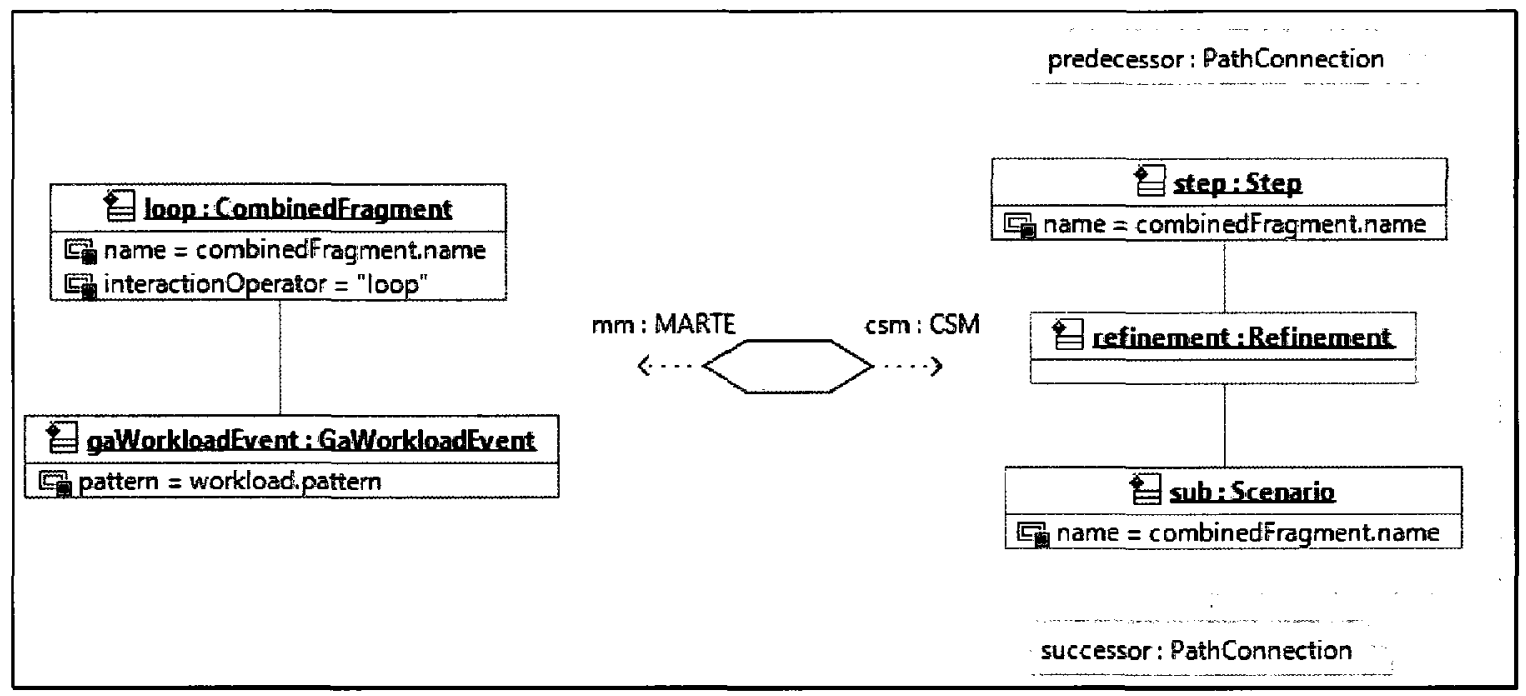

Figure 3-17: Loop Fragment - Transformation Rule

Loop fragments are transformed in to steps with their own Refinements, linking to a subscenario, each beginning and ending with a special Starts and Ends, respectively.

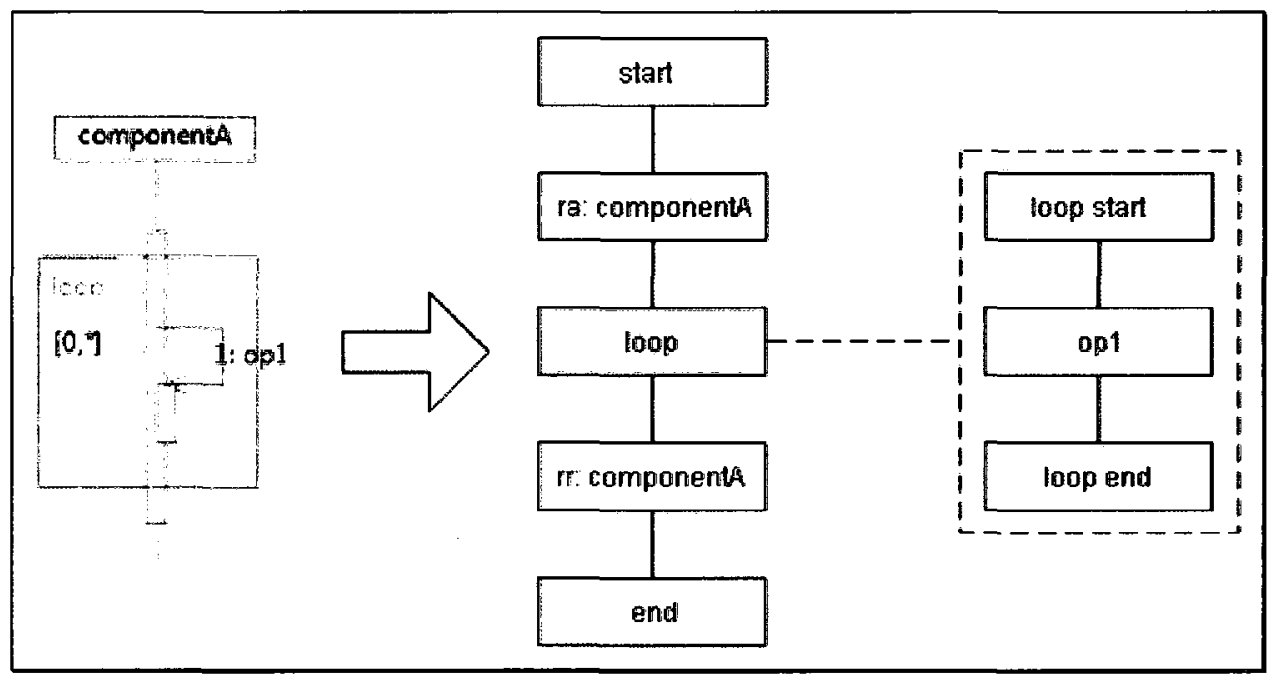

Figure 3-18: Loop Fragment - Transformation Example 


\section{Chapter 4. Design Patterns}

\subsection{Two-tier Rule Approach}

\subsubsection{Goal}

To create transformation rules for source elements that may have more than one mapping, some of which may be conditional on a set of constraints.

\subsubsection{Motivation}

One of the key aspects of designing a transformation of any model to another, especially when using a rules-based transformation language, is matching elements from the source model with those of the target model. Some of those mappings are unconditional, with the source elements always mapping to the same target elements no matter the circumstances. Other mappings are conditional and seem to rely on a special set of circumstances surrounding each source element.

Like the Duality Pattern previously described in section 2.5.2, the proposed Two-tier pattern is targeted towards those elements that have dual characters. The main difference between the patterns however is that in the Duality Pattern, independent transformation rules are created for each of these cases. The Two-tier approach removes much of this redundancy by having separate rules for target elements generated from unconditional mappings and those from conditional mappings.

\subsubsection{Specification}

In cases where mappings are unconditional, automatic deterministic logic should be employed to execute the transformation. Mappings that exist on conditional logic should have 
imperative rules that are explicitly invoked by the former. Figure 4-1 illustrates the composition of a transformation model following the Two-tier approach:

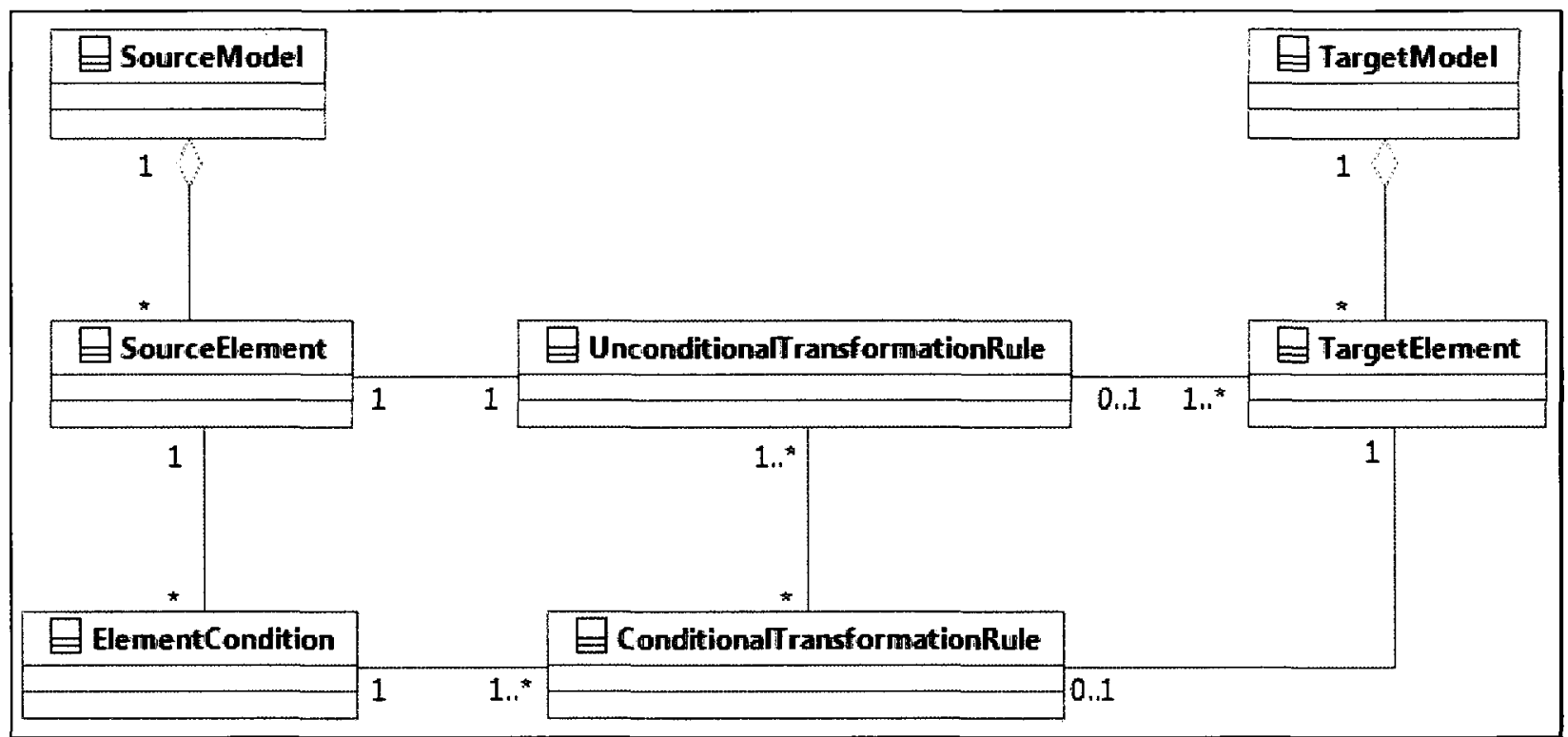

Figure 4-1: Two-tier Rule - Generic Transformation Structure

\subsubsection{ATL Solution}

ATL provides two different types of rules that can be used in each of the different types of mappings described above, matched and lazy rules. Matched rules automatically generate all specified target elements from a source element during the execution of a transformation. These types of rules are best used for unconditional mappings between source and target models. The other type of rule, lazy, must be called imperatively from another rule. This kind of rule is suitable for conditional mappings.

Figure 4-2 demonstrates the ATL-specific implementation of the Two-tier approach, with both matched and lazy rules being employed: 


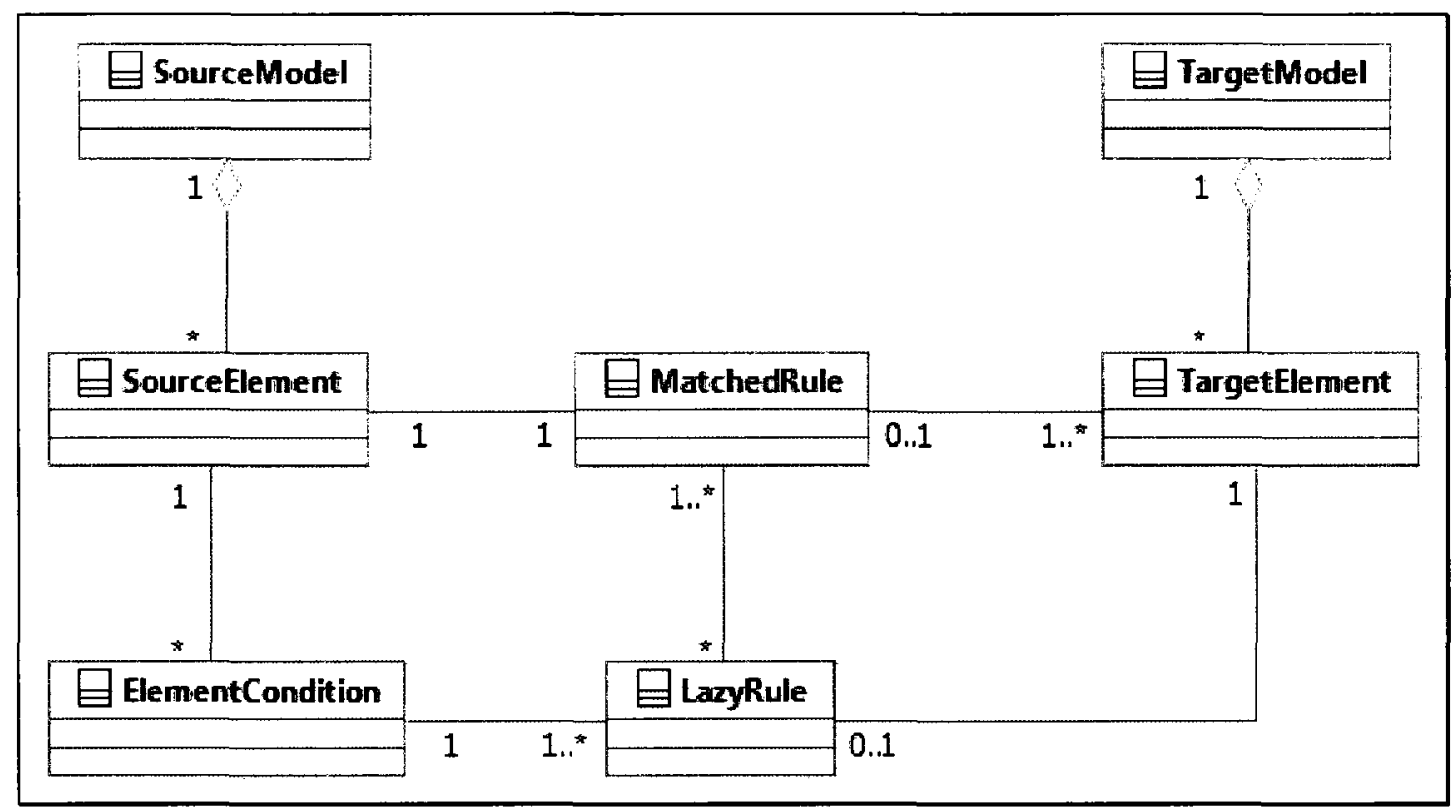

Figure 4-2: Two-tier Rule - ATL Transformation Structure

All SourceElements from the SourceModel associated with a MatchedRule will automatically be transformed in to corresponding TargetElements in the TargetModel. Any ElementCondition that the source element has will cause an invocation of a Lazy Rule, from the MatchedRule, generating the specified TargetElements.

\subsection{Relative Link Pattern}

\subsubsection{Goal}

To ensure proper linkage of elements in target models of model transformations.

\subsubsection{Motivation}

A key requirement of transformation models is that elements in a target model must correctly reference each other in a way relative to how their corresponding elements in the source model do. Drawing upon the Mapping Pattern described in section 2.5.3, is the Relative 
Link Pattern, which specifies how target elements are linked with one another. The logic is based on the relationships their source model counterparts have.

\subsubsection{Specification}

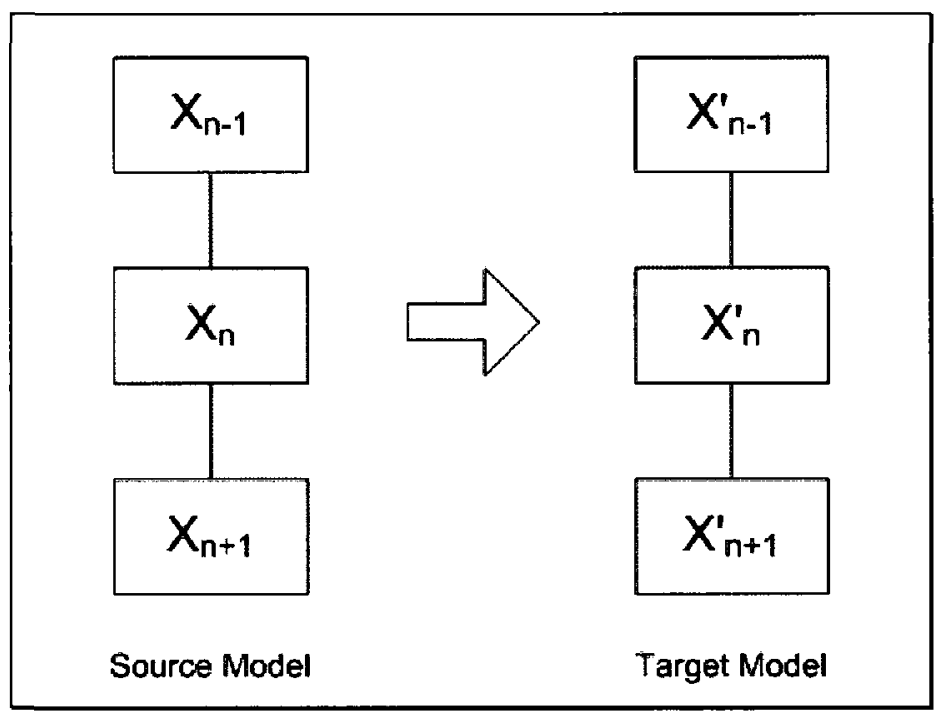

Figure 4-3: Relative Relationships of Source and Target Models

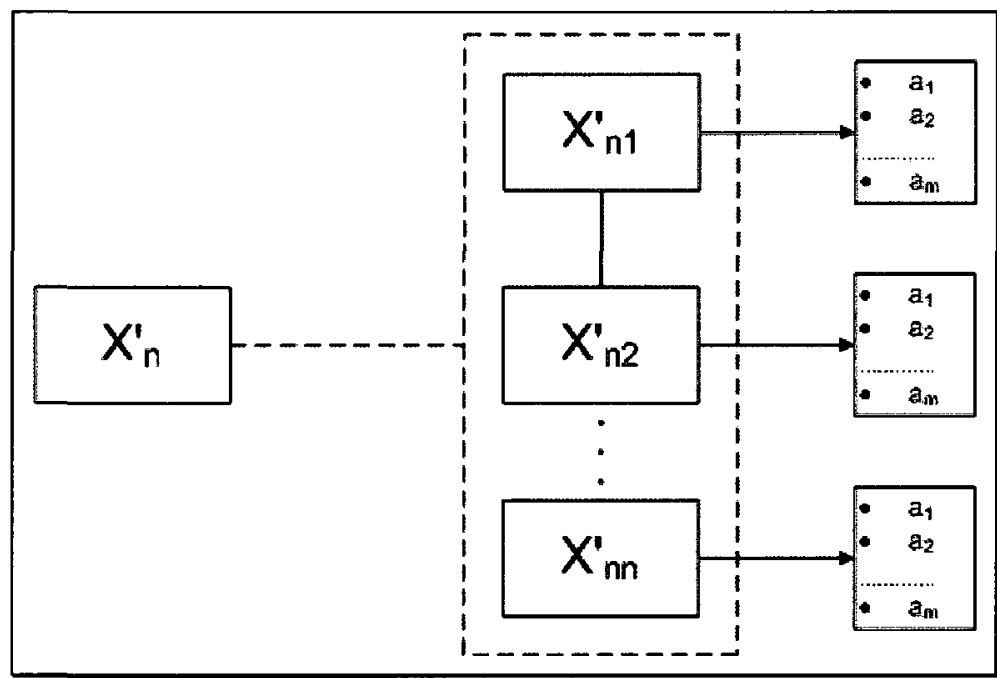

Figure 4-4: Target Model Sub-graph 
As shown in Figure 4-3, if an element $x_{n}$ has $x_{n-1}$ and $x_{n+1}$ as linked elements, then $x_{n}^{\prime}$ must have $\mathrm{x}_{\mathrm{n}-1}^{\prime}$ and $\mathrm{x}_{\mathrm{n}+1}^{\prime}$ as linked elements. Also, each target element, $\mathrm{x}_{\mathrm{n}}$, may represent more than one sub-element (as shown in Figure 4-4), whereby the sub-elements $\mathrm{x}_{\mathrm{n} 1}^{\prime}$ and $\mathrm{x}_{\mathrm{nn}}^{\prime}$ would be be linked to $\mathrm{x}_{\mathrm{n}-1}^{\prime}$ and $\mathrm{x}_{\mathrm{n}+1}^{\prime}$, respectively. Each sub-element's attributes are represented by the set $\left\{\mathrm{a}_{1}, \mathrm{a}_{2}, \ldots, \mathrm{a}_{\mathrm{m}}\right\}$.

\subsubsection{ATL Solution}

In ATL, each rule should be modeled according to Code Fragment 4-1. With the first target element, $x_{n 1}^{\prime}$, a series of if statements are made to check what condition, $i$, the source element, $x_{n}$, or its linked element, $x_{n-1}$, satisfies. Depending on which condition is satisfied, the first target element references its linked element by binding its $x_{n 1}^{\prime} a_{1}$ attribute to the appropriate rule. The other attribute, $b_{1} a_{2}$, usually binds to the next target element in the rule. However, if there is only one target element in the rule, $b$, then $x_{n 1}^{\prime} a_{m}$ follows the same logic of the last element attribute shown in Code Fragment 4-1, $\mathrm{x}_{\mathrm{nn}}^{\prime} \mathrm{a}_{\mathrm{m}}$.

In the example of Figure 3-7, if a reflexive message is first in a lifeline, the resultant Step will be preceded by a PathConnection joining it with a ResourceAcquire.

For the last target element, $\mathbf{x}_{\mathrm{n} n}^{\prime}$, a series of if statements are made to check what condition, $i$, the source element, $x_{n}$, or its linked element, $x_{n+1}$, satisfies. Depending on which condition is satisfied, the last target element references its linked element by binding its $x_{n n}^{\prime} a_{m}$ attribute to the appropriate rule.

In the example, if a reflexive message is last in its lifeline, the resultant Step will be succeeded by a PathConnection joining it with a ResourceRelease. 
Code Fragment 4-1 is the algorithm used in each ATL rule to properly link target elements with one another to reflect the linked structure of their source model counterparts.

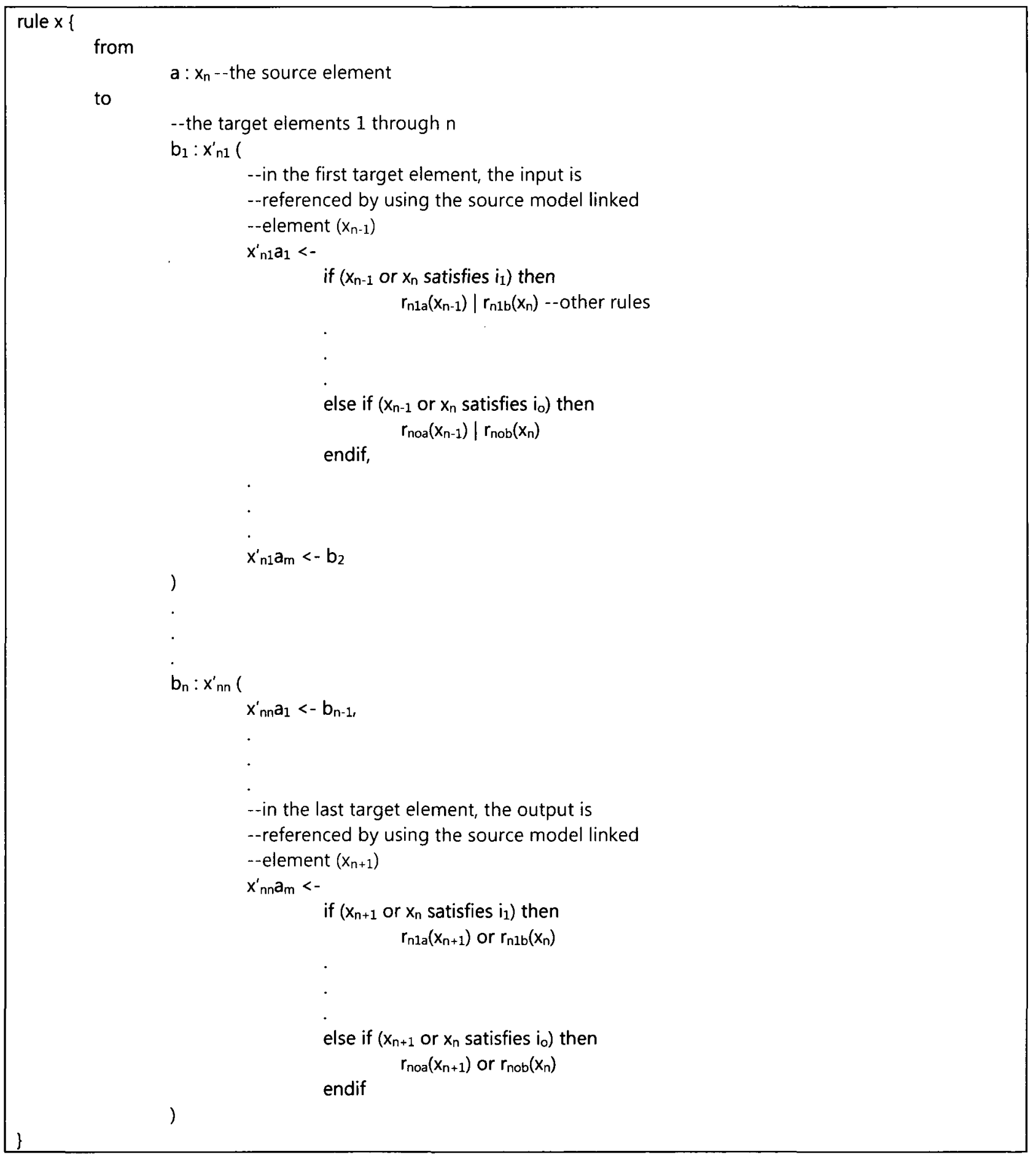

Code Fragment 4-1: ATL Algorithm for Relative Link Pattern 


\subsection{Containment Pattern}

\subsubsection{Goal}

To ensure that target models of rule-based transformation models conform with their respective metamodels in terms of structure.

\subsubsection{Motivation}

Another concern when designing a transformation model is the structure of the target model. Ultimately, one target element will become the root container of the target model, while others will become sub-containers or leaf elements. In order to have certain target elements be contained with another element, the transformation rule that generates the container must reference the rule that generates the child target element.

\subsubsection{Specification}

Figure 4-5 illustrates the logic behind containment in the rules-based paradigm.

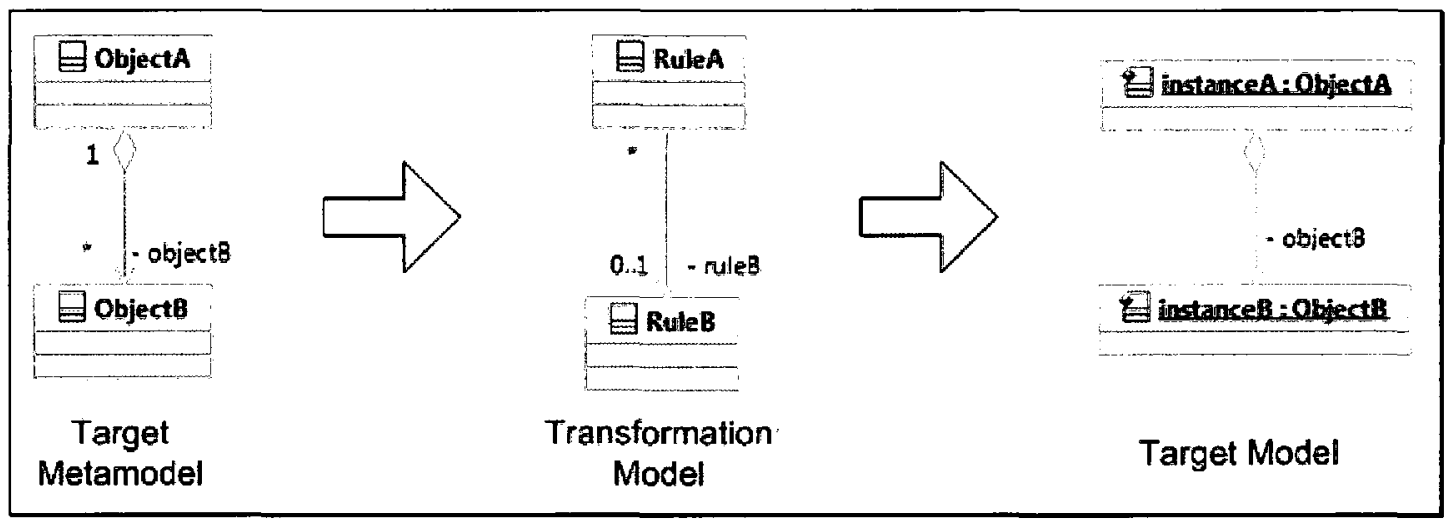

Figure 4-5: Containment Pattern

In this case, instance $A$ in the target model encapsulates a collection of instanceB by referencing the transformation rule, RuleA. If RuleA did not reference the objects from RuleB, 
all target elements would appear as one large flattened collection. Therefore, designing a rulebased algorithm around the containment hierarchy of the target metamodel is crucial in order to produce consistent target models.

\subsubsection{ATL Solution}

The ATL language provides facilities for automatically defining the attributes of target elements, even when those attributes are references. The module function, "resolveTemp", binds an attribute of a target element to another target element generated by another rule. Assuming the target metamodel of Figure 4-5 has an identical source metamodel, its implementation in ATL gives the transformation model in Code Fragment 4-2.

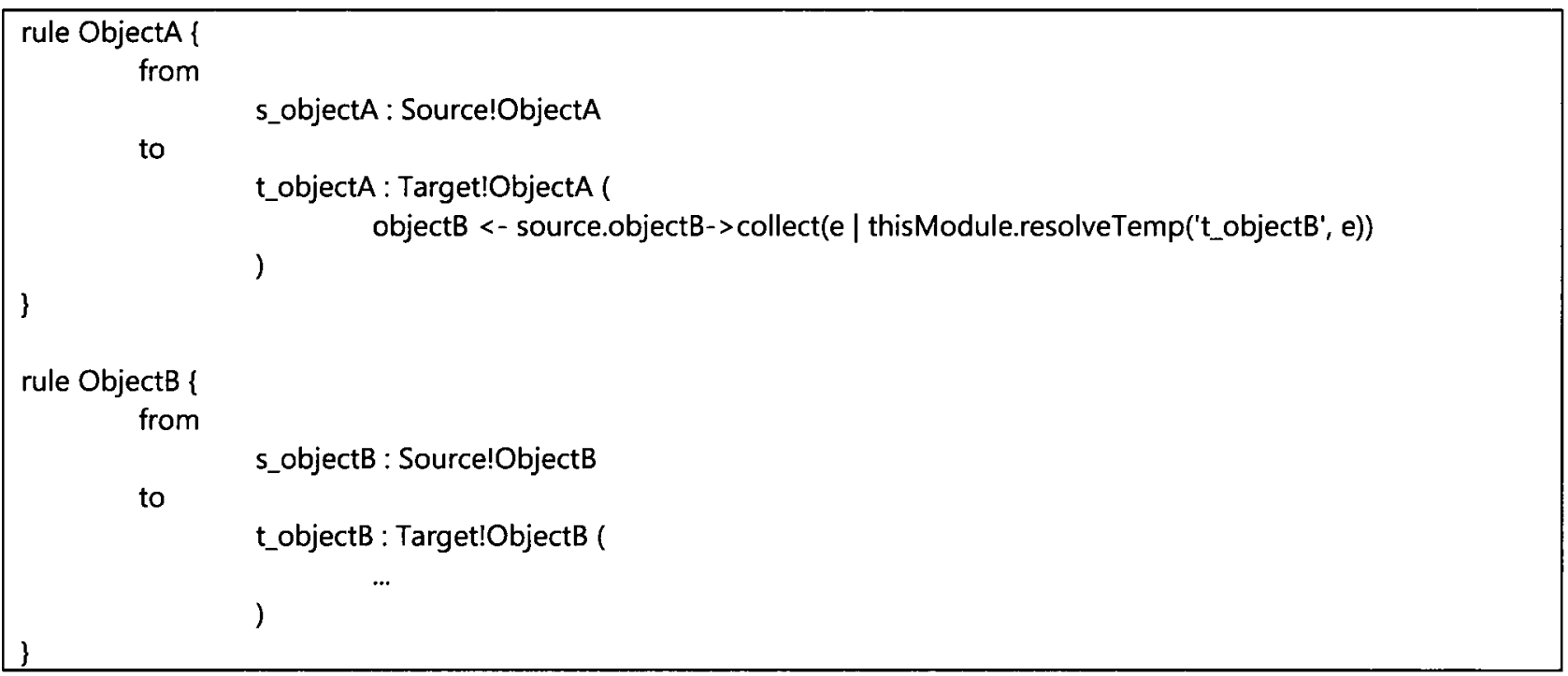

\section{Code Fragment 4-2: ATL Implementation of Containment Pattern}

By binding the target element, $t$ _objectB, to the attribute objectB of $t$ _objectA, the ATL rule ensures that objectB will be encapsulated by objectA in the target model after execution.

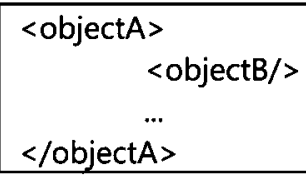

Code Fragment 4-3: XMI of Target Model Following Application of Containment Pattern 


\section{Chapter 5. Implementation}

\subsection{Transformation Model}

\subsubsection{Architecture}

The ATL implementation of the transformation in this study follows the same structure as all other ATL transformations. The source, transformation, and target models each have their own separate metamodels, which are each based on a common meta-metamodel (ECORE).

The source metamodel is the UML2 EMF library included in the Eclipse environment, while the target metamodel is custom written in ECORE. The transformation module, MM2CSM, conforms to ATL and makes use of a library of helpers called MM2CSM Lib. MM2CSM accepts a UML+MARTE source model, exported from RSA, and produces a CSM. Both the source and target models are expressed using the XMI storage facility.

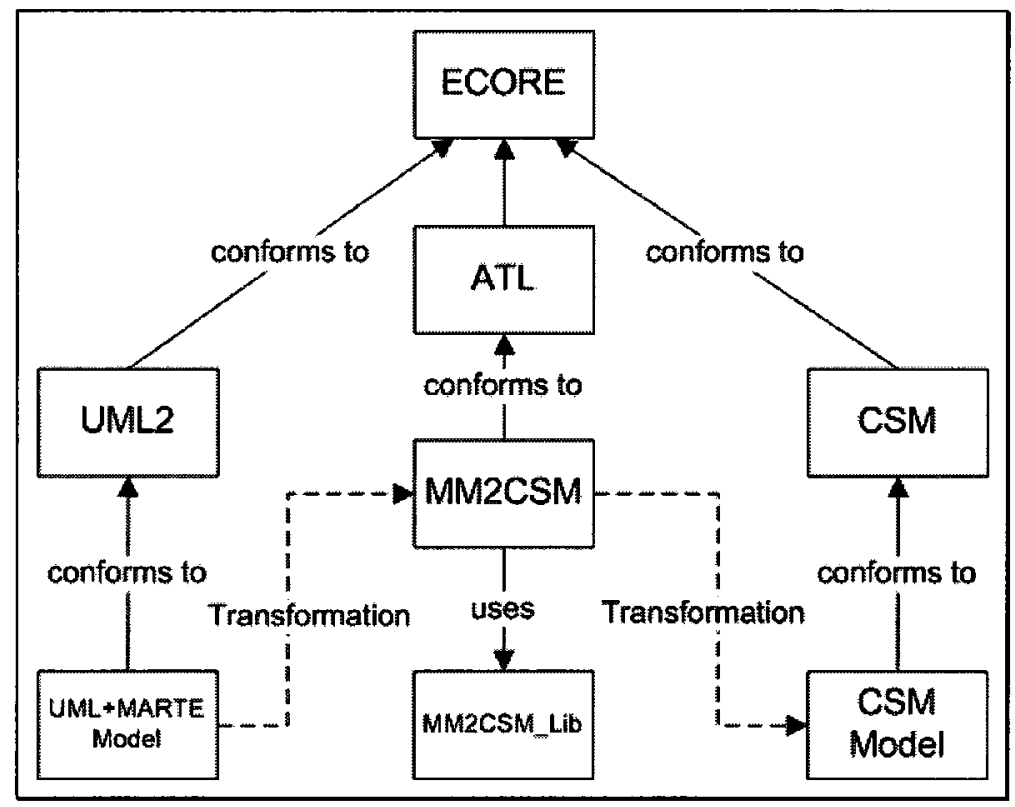

Figure 5-1: UML+MARTE Interaction Diagram to CSM ATL Model 
When the transformation architecture of a typical ATL model, presented in Figure 2-7, is applied to this specific domain and implementation, the illustration in Figure $5-1$ is conceptualized.

\subsubsection{ATL Module}

Within the ATL module containing all the transformation rules, MM2CSM, are 12 matched rules for all conditional mappings (see Table 3-1), and 30 lazy rules for all unconditional mappings (see Table 3-2). This implementation follows the ATL-specific approach of the Two-tier Rule Approach described in section 4.1.2. Matched Rules are used for source elements such as interactions, messages, fragments, and certain applied MARTE stereotypes, whereas lazy rules are used for source elements that satisfy specific conditions generating target elements such dummy steps, starts, ends, forks, joins, or any needed step sequences.

The logic linking each target elements with one another, based on the ordering of the source model's elements, is embedded within each rule. Using helpers defined in MM2CSM_Lib, each rule determines what the preceding and succeeding elements of the transformation candidate is. These helpers do this by traversing the ordered list of fragment elements (see Figure 3-3) in the source model. Then by using the Relative Link pattern detailed in section 4.2.2, rules can be implemented in such a way that target elements are properly linked with each other when generated.

Finally, the Containment pattern (of section 4.3.1) is employed in MM2CSM by binding the attribute of each target element specified within rules with the proper target element of other rules. Figure 5-2 displays the matched rule domain of MM2CSM. Since the Model rule element 
("csm" in the diagram) contains the scenarios, workloads, components and refinements as children; the rule binds those target elements, wherever they are generated, to the attributes of "csm". Likewise, the scenario element in the Interaction rule binds all generated steps and path connections to the scenario's attributes, make them children to the scenario.

Following these three patterns ensure that the ATL transformation model has: a) sourceto-target rules based on model mappings (laid out in the Chapter 3); b) elements in the target model that are properly linked with one another; and c) a target model that conforms to the CSM metamodel.

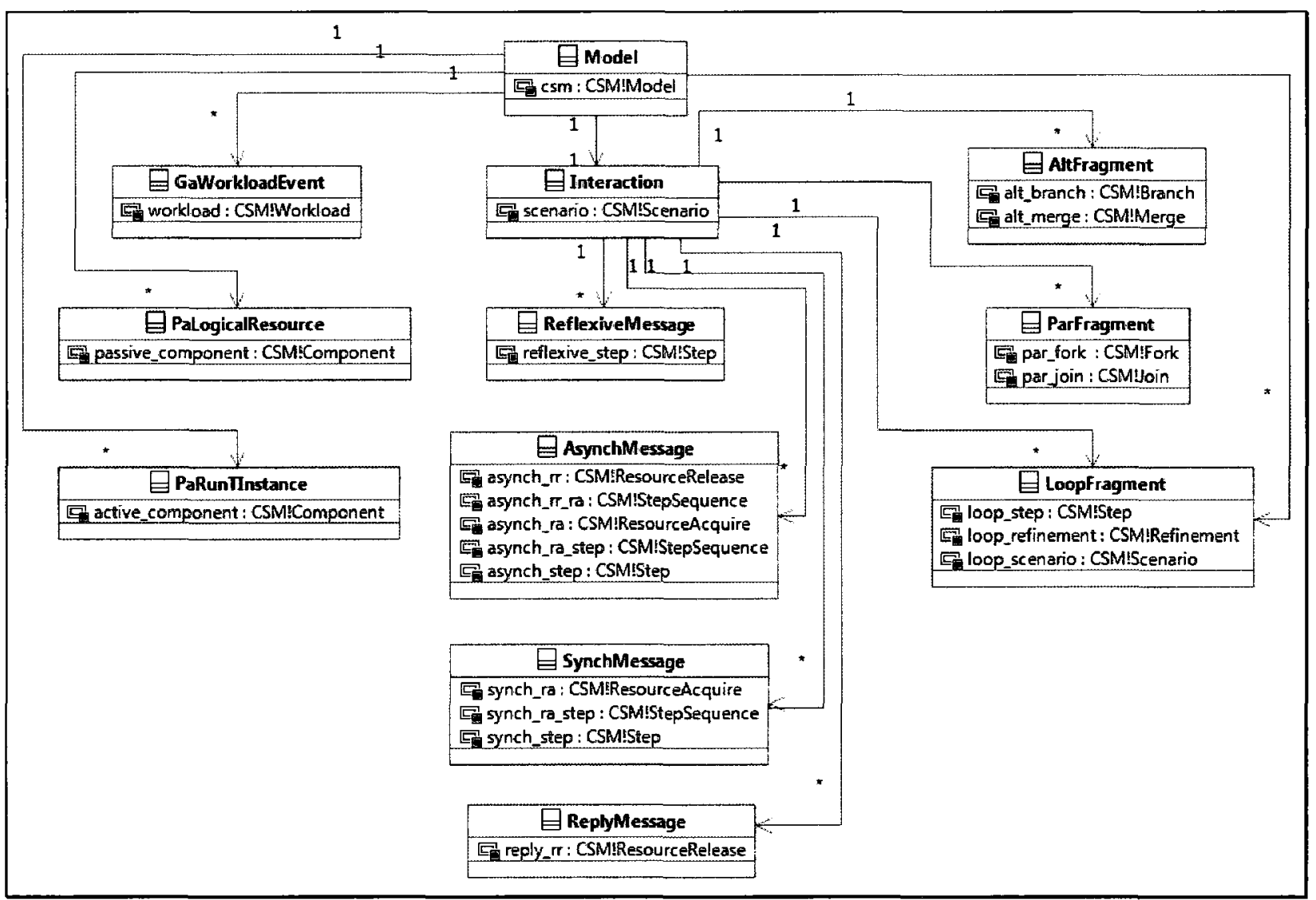

Figure 5-2: Matched Rule Domain of MM2CSM 
In Code Fragment 5-1, the ATL matched rule for transforming UML Models in to CSMs is demonstrated. Since both the source and target element are the root containers in their respective models, the Containment Pattern is followed in this rule, with each of the target elements derived from the UML Model being bound to the CSM's attributes.

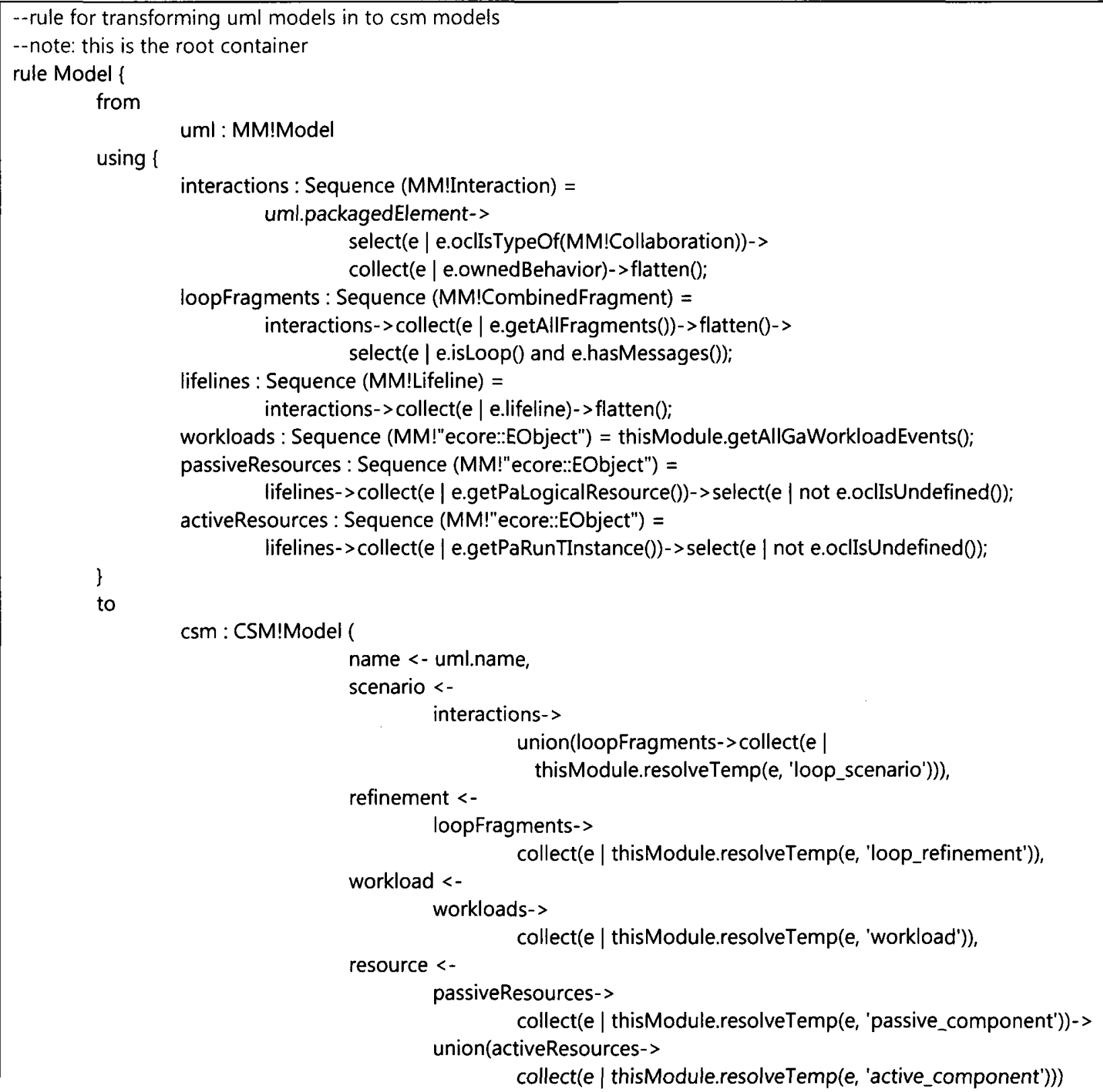

Code Fragment 5-1: ATL Rule for Models 
As an example of the many helpers that exist in the MM2CSM_Lib module, the getPrevMessageInLifeline() helper is demonstrated in Code Fragment 5-2. Using OCL syntax, this helper takes the sender lifeline of the message in context and returns the previous message (whether it is being sent or received). If the message in context is the first message in the lifeline, then a null value (specified by "OclUndefined") is returned.

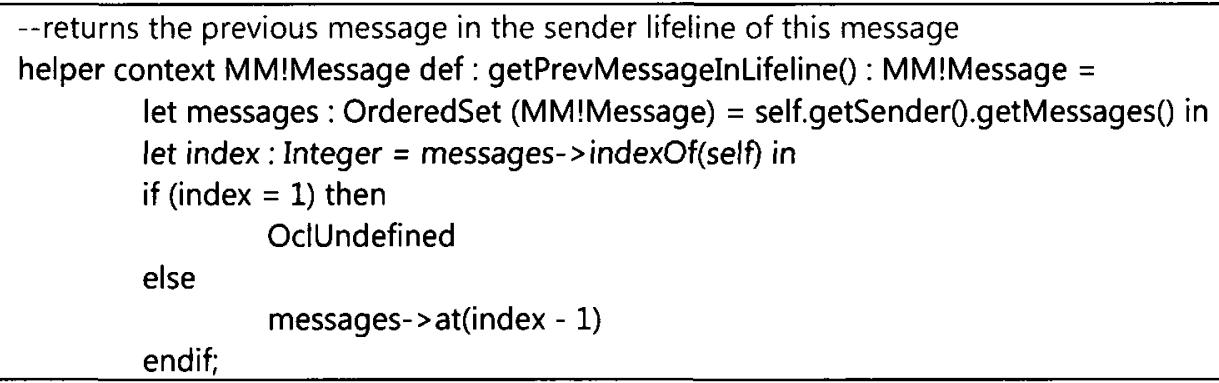

\section{Code Fragment 5-2: ATL Helper for Getting Previous Message}

Below, Code Fragment 5-3 contains the code for an ATL matched rule for synchronous messages. The Relative Link Pattern is followed in this rule, with each of the target elements being linked to other elements based upon the source model's structure. Of particular note are the predecessor attribute of "synch_ra" and successor attribute of "synch_step". In each case, a thorough check through all possible scenarios is made to determine what other target elements are to be linked.

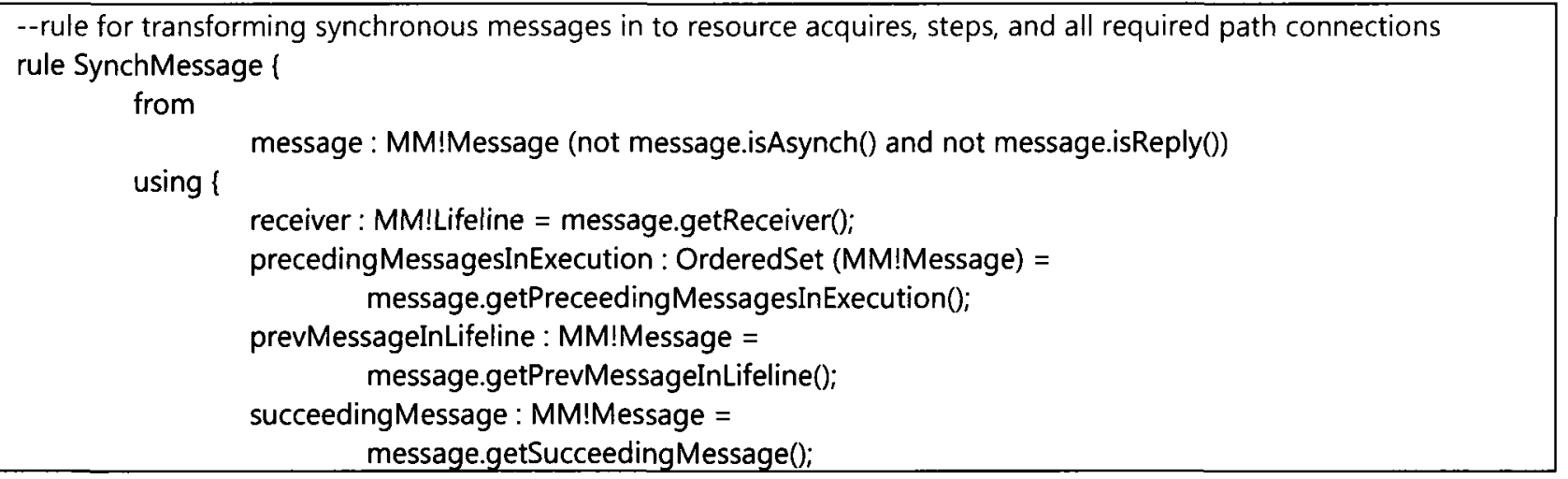

Code Fragment 5-3: ATL Rule for Synchronous Messages 


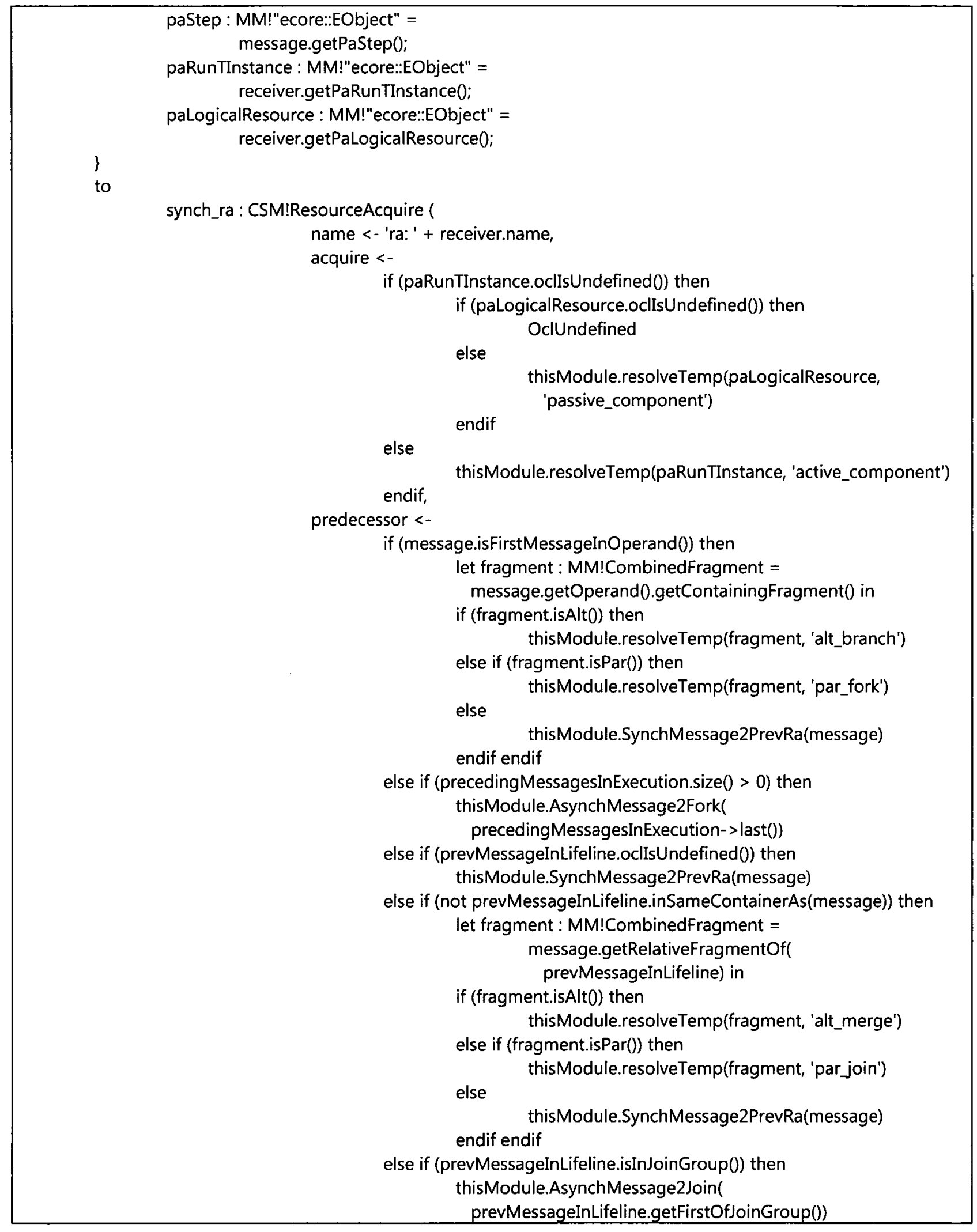

Code Fragment 5-3: ATL Rule For Synchronous Messages (cont'd) 


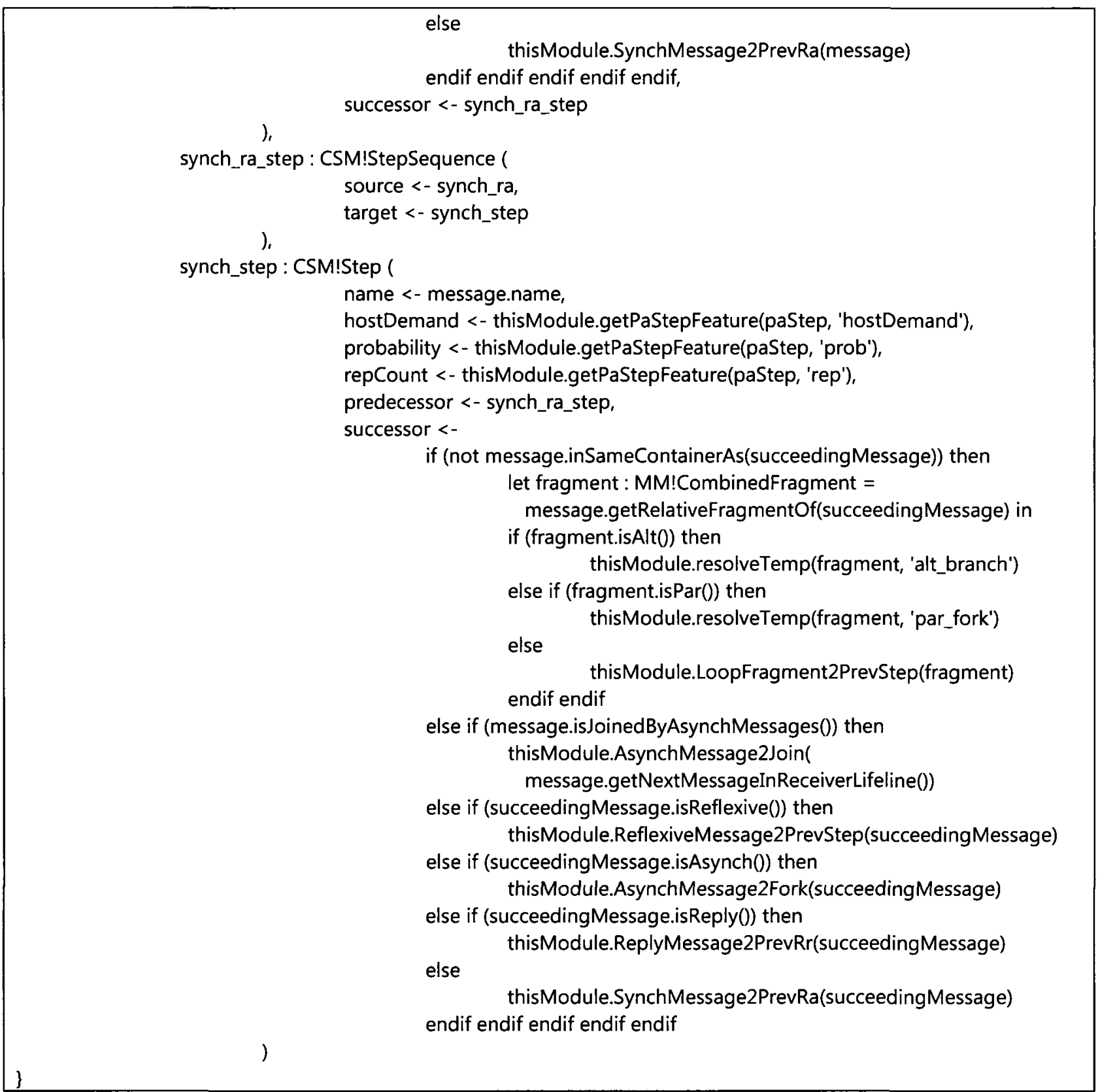

\section{Code Fragment 5-3: ATL Rule For Synchronous Messages (cont'd)}

\subsection{Transformation Process}

The entire transformation process of a UML+MARTE Sequence Diagram to a CSM can be described in 5 basic steps: 
1. Using RSA or the RSA view in Eclipse, a UML+MARTE Sequence Diagram with applied MARTE stereotypes is created.

2. While still in RSA, the UML model is exported to XMI form.

3. In Eclipse, a transformation run configuration, which specifies both the XMI file as the source model and the destination of the target model, is created.

4. The ATL transformation run configuration is executed.

5. The generated XMI target model is loaded in to the CSM Visualizer, which produces a visualization of the target model.

Below, Figure 5-3 illustrates a workflow of these steps in addition to the context of each action, as well as the tool involved, whether it's RSA, ATL or the CSM Visualizer.

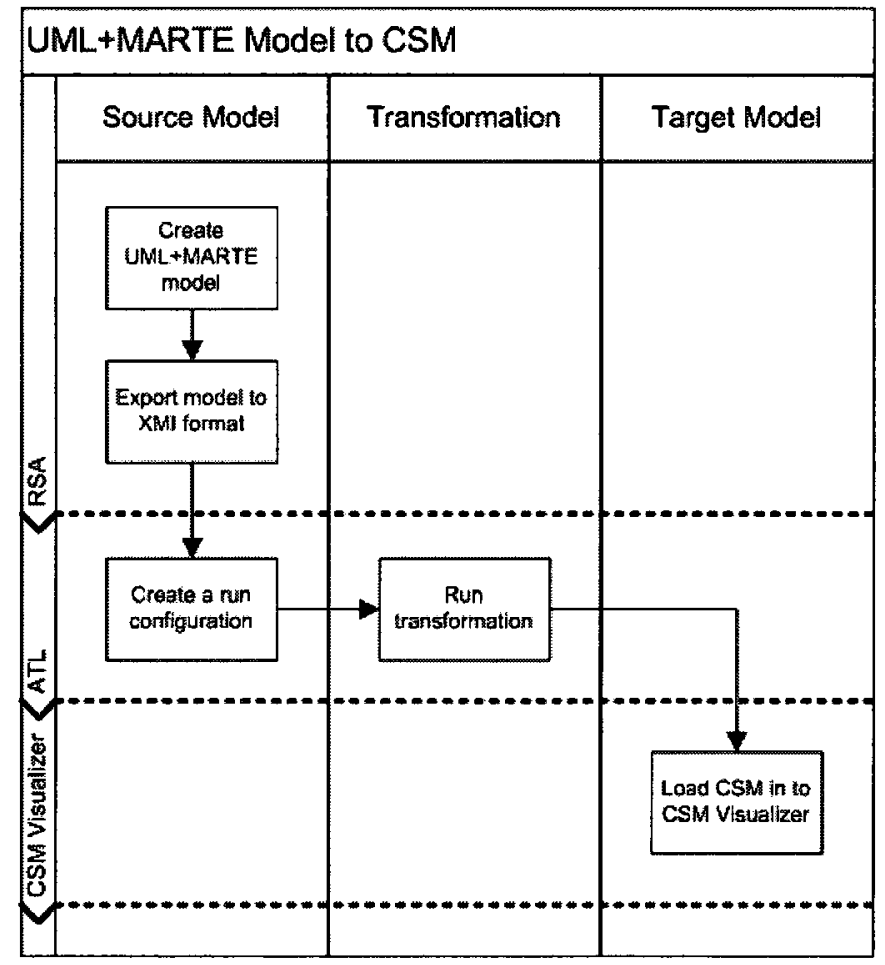

Figure 5-3: User Steps in Transformation Process 


\subsection{Output Visualization}

The target model produced by the ATL transformation is in XMI form. Although this is adequate for the purpose expressing a CSM, it makes human verification a labour-intensive process. To solve this shortcoming, part of this study included the development of a utility that visualizes generated CSMs from the transformation.

The two chief requirements of the CSM visualization tool were: accepting the XMI target model as input and parsing its XML contents; using the parsed elements to produce graphic shapes and labels that constitute a CSM diagram.

Fortunately, the J2SE API and its extensive third-party libraries provided the necessary facilities for satisfying these requirements [Java]. The Apache Xerces XML Parser includes libraries that allow the parsing of elements and their attributes within any well-formed XML file [Xerces]. Also, the native javax.swing libraries of J2SE include adequate tools for visualizing the target model.

As a first step, the CSMReader class reads the XMI file, creating a Model. The Model class is a object abstraction of the CSM complete with Scenarios, Steps and PathConnections referencing each other each other as defined in the XMI file. Both Steps and PathConnections are subclasses of the ScenarioElement class.

Using the Model instance, an actual Diagram of the CSM can be constructed. Diagrams have a fairly simple composition. They consist of Nodes residing on Rows connected to Nodes on preceding or succeeding Rows. Displayed below, in Figure 5-4, is a CSM diagram generated by a transformation from the interaction example in Figure 3-2. 


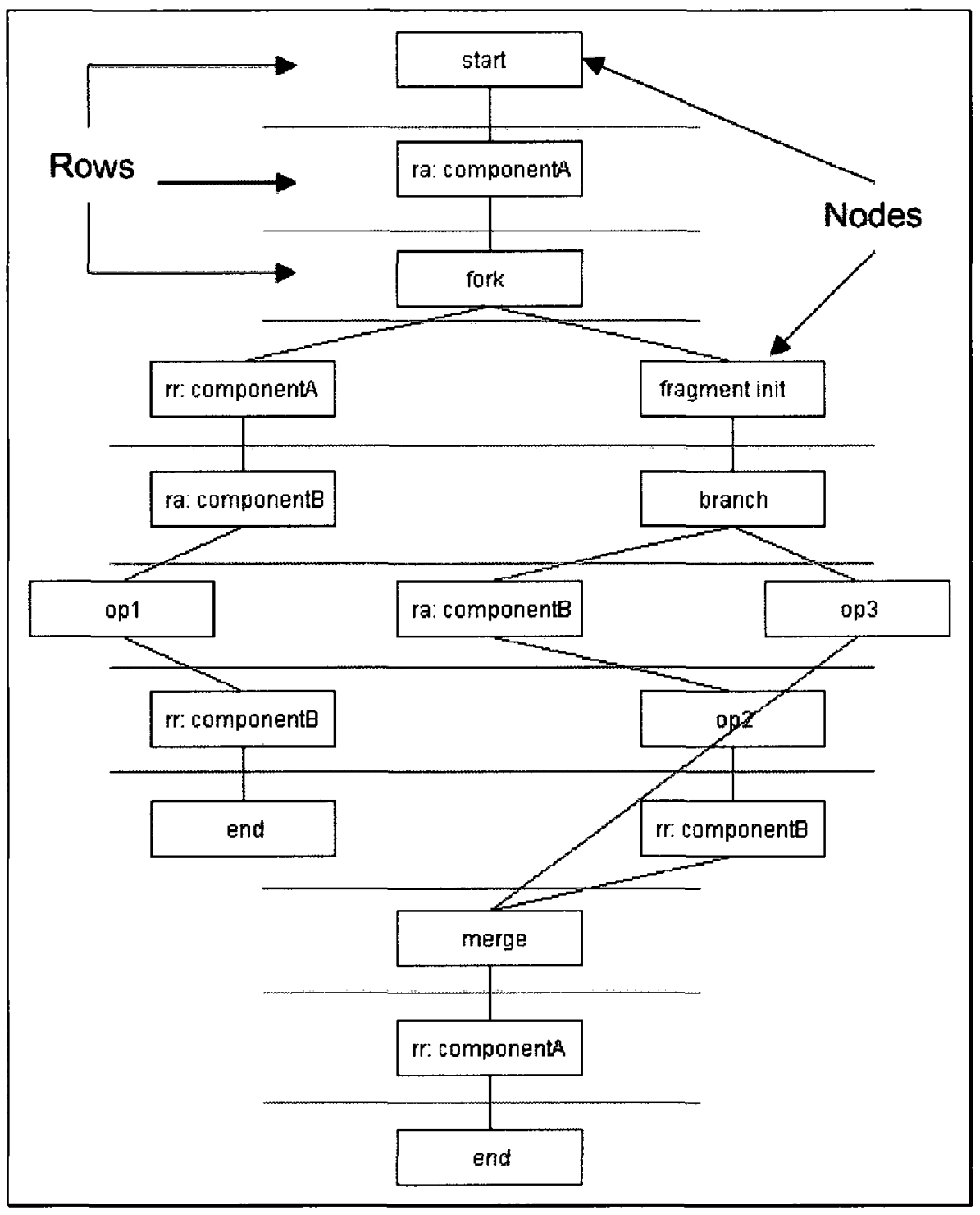

Figure 5-4: CSM Diagram Elements

The conversion of a Model in to a Diagram requires a complex algorithm that's a variation of the Breadth-First Search graph traversal algorithm. First though, it must be established that all StepSequence PathConnections, which simply link Steps to their succeeding Step, do not become nodes on graphs. This is in contrast to Fork, Join, Branch and Merge PathConnections, which all link to several Steps. 
As in BFS, the algorithm starts at the root of the graph (in this case the start Step of the CSM) and traverses each successor, while inserting a node in to each row of the diagram. However, there are occasions where the successor element being traversed has already had a corresponding node inserted in to a row that's at a level preceding the current element (like with nodes "rr:componentB" and "merge" in Figure 5-3). In these cases, the nodes and each of their successors are promoted to a new row with a recursive function (entitled move_to_a_new_row(..) in Code Fragment 5-4). The pseudocode in Code Fragment 5-4 describes the algorithm of constructing a CSM Diagram. Its implementation is contained within the Diagram class.

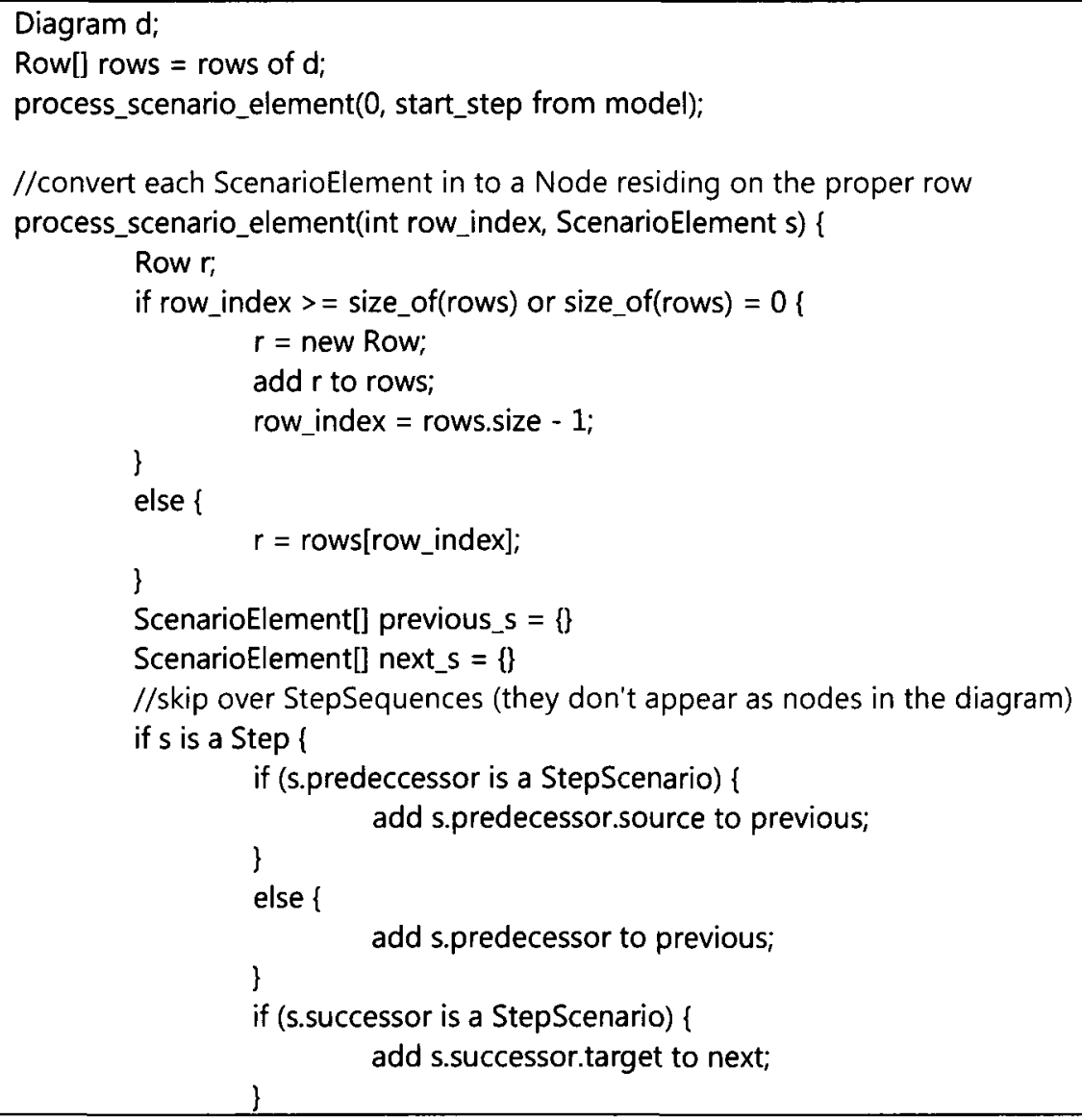

Code Fragment 5-4: Algorithm for Constructing CSM Diagram 


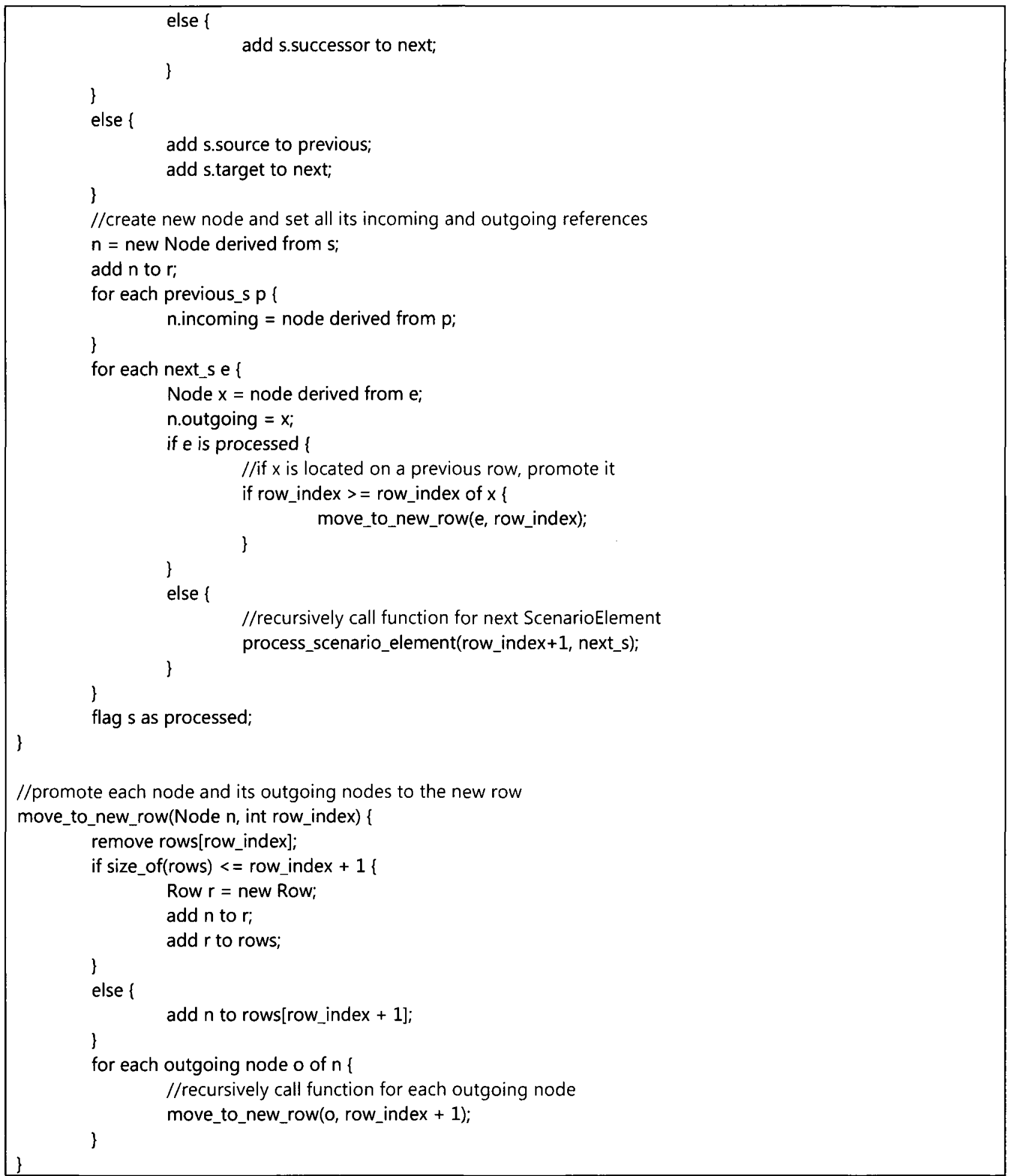

Code Fragment 5-4: Algorithm for Constructing CSM Diagram (cont'd)

The generated class diagram of Figure 5-5 details each of the Java classes within the utility, their fields, and their relations to one another. 


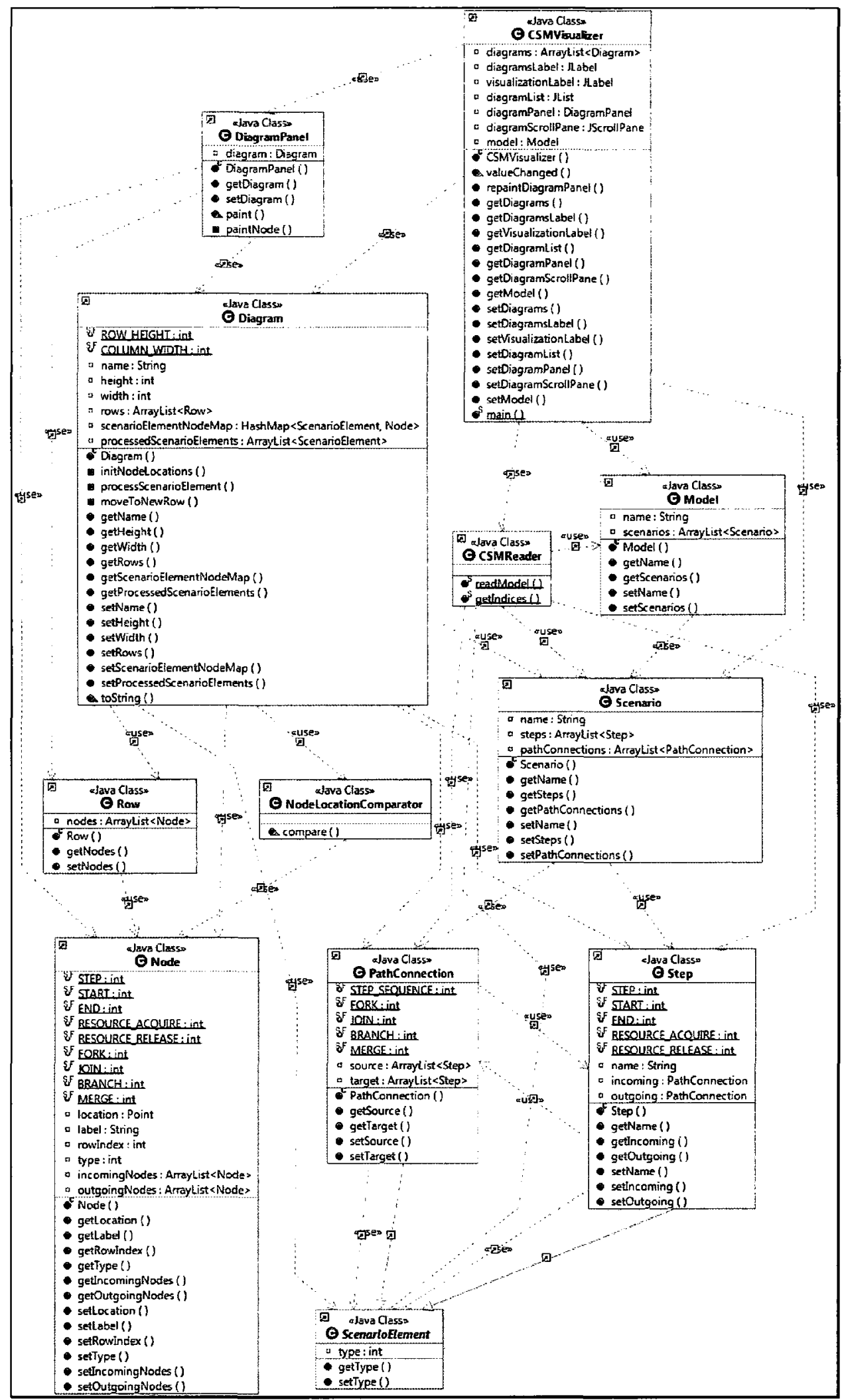

Figure 5-5: CSM Visualizer Java Class Diagram 


\section{Chapter 6. Verification and Case Study}

\subsection{Checklist}

In this chapter is described the verification of the ATL transformation with the help of several test cases. Each test case was designed to cover a number of special conditions, as laid out in the checklist from Table 6-1. These conditions primarily involve complex circumstances that require the invocation of one of the conditional mapping rules described in section 3.2 of this study. By verifying the correctness of the test case results, we can also ascertain the correctness of the unconditional mapping rules, since each of the lazy rules invoked in ATL are also referenced by matched rules. Each test case contains one or more of these conditions, adding extra layers of complexity to their transformations. The XMI form of each CSM can be found in the appendix.

\begin{tabular}{|c|c|c|c|c|c|c|}
\hline & 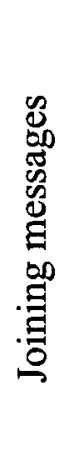 & 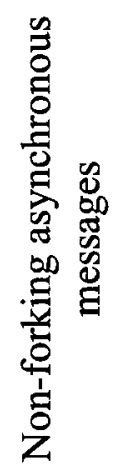 & 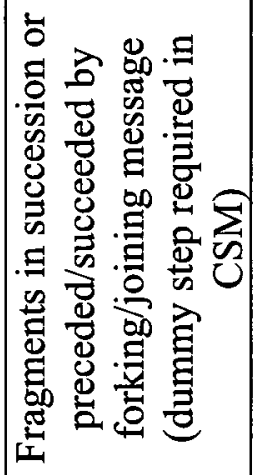 & 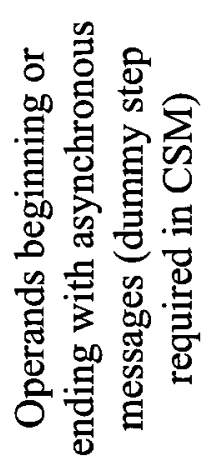 & 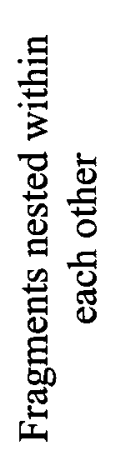 & 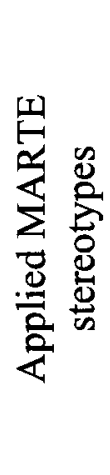 \\
\hline TC \#1 & * & & & & & \\
\hline $\mathrm{TC} \# 2$ & & $*$ & & & & \\
\hline TC \#3 & & & $*$ & * & & \\
\hline $\mathrm{TC} \# 4$ & & & $*$ & & & \\
\hline TC \#5 & & $*$ & & * & * & \\
\hline TC \#6 & $*$ & $*$ & & & $*$ & \\
\hline PUMA & & & & $*$ & $*$ & \\
\hline$\pm \mathrm{VAS}$ & * & & & & & $*$ \\
\hline
\end{tabular}

Table 6-1: Test Case Checklist 


\subsection{Tests}

\subsubsection{Test Case \#1}

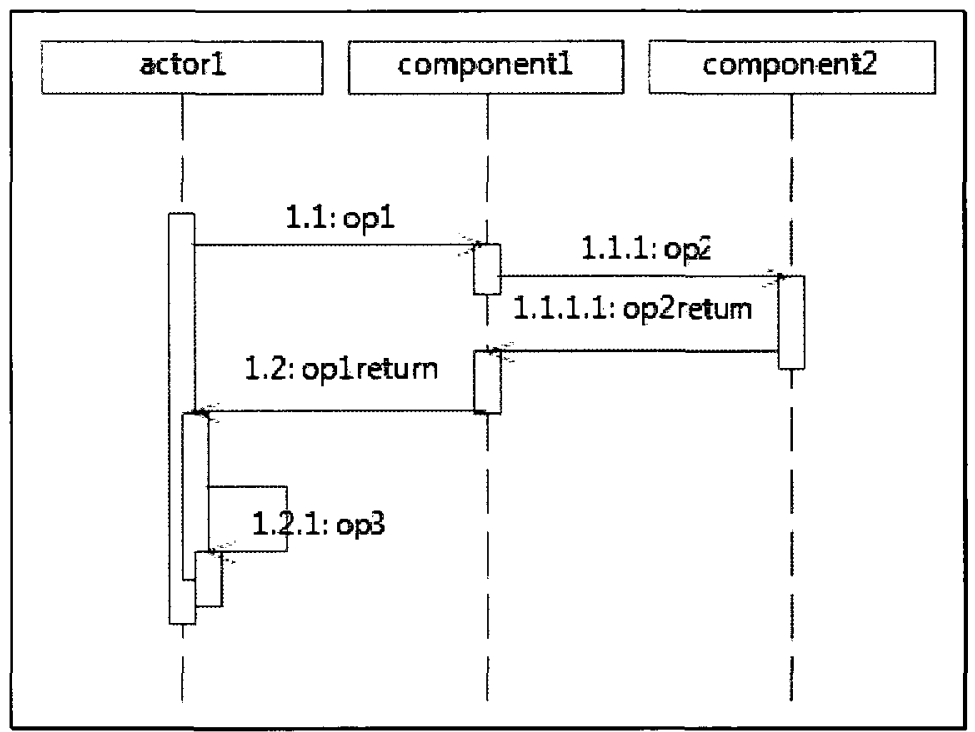

Figure 6-1: Test Case \#1 - Source Model

In this scenario, the transformation of join messages is tested. The asynchronous message "oplreturn" joins a behaviour specification thread with an outgoing message "op1" preceding it. In the CSM, the fork that occurs before "op1" leads to a path that later joins with "op1return." Also, since forks cannot be succeeded by another PathConnection, a dummy step must be inserted after the fork (in this case called "join init"). Succeeding the join is the step "op3," transformed from the reflexive message in the source model. 


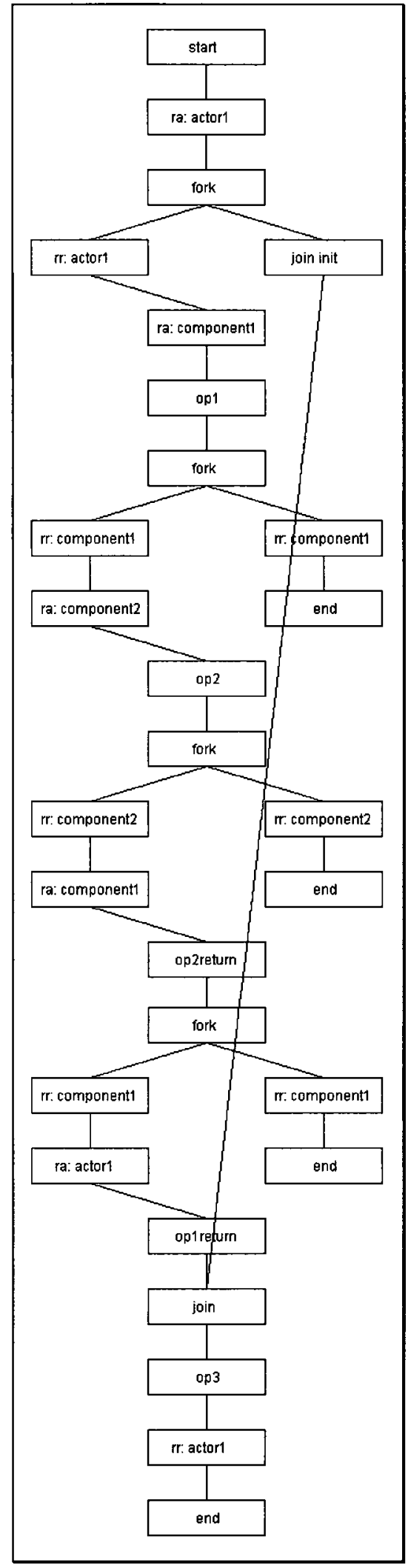

Figure 6-2: Test Case \#1 - Target Model 


\subsubsection{Test Case \#2}

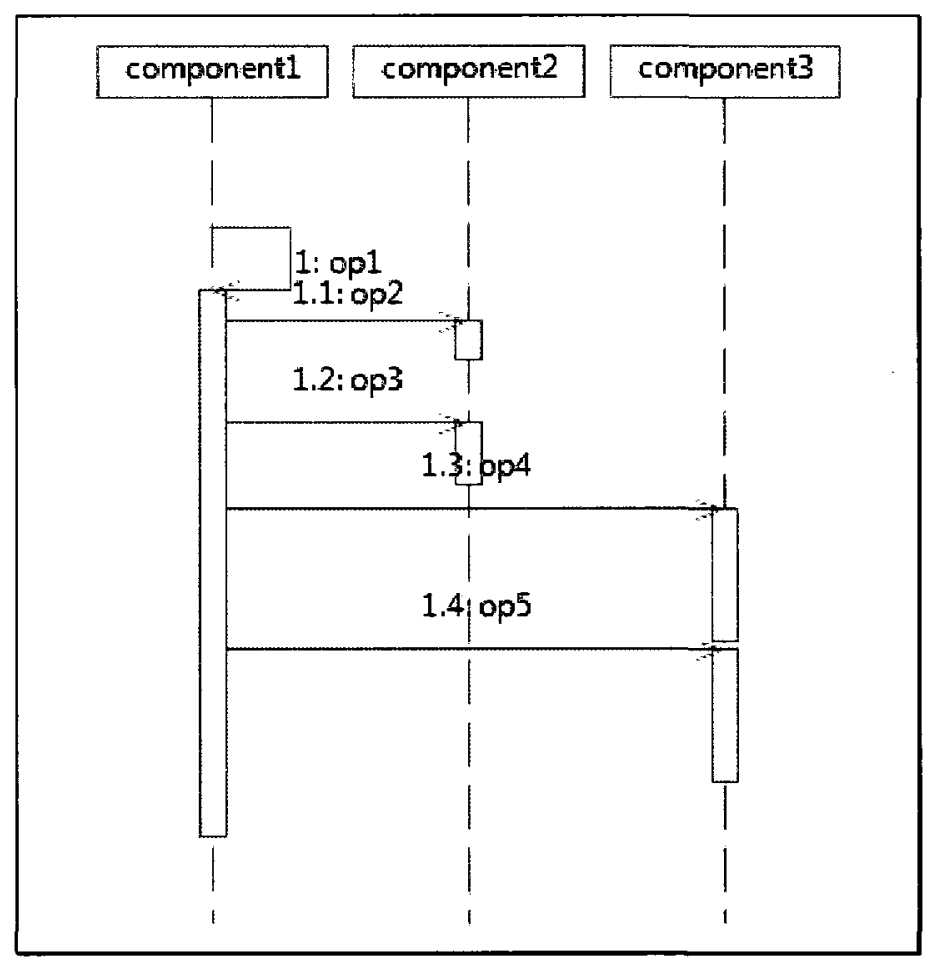

Figure 6-3: Test Case \#2 - Source Model

This case tests the proper transformation of asynchronous messages in to forks. Asynchronous messages lead to one fork if there are no preceding asynchronous messages in the sender lifeline. In this scenario, the message "op2" is preceded by only one reflexive message, "op1" and is succeeded by 3 other asynchronous messages, "op3," "op4," and "op5" respectively. In the resultant target model, only one fork is generated and its target links are "op2," "op3," "op4" and "op5," demonstrating the generation of forks occurred under the right condition. 


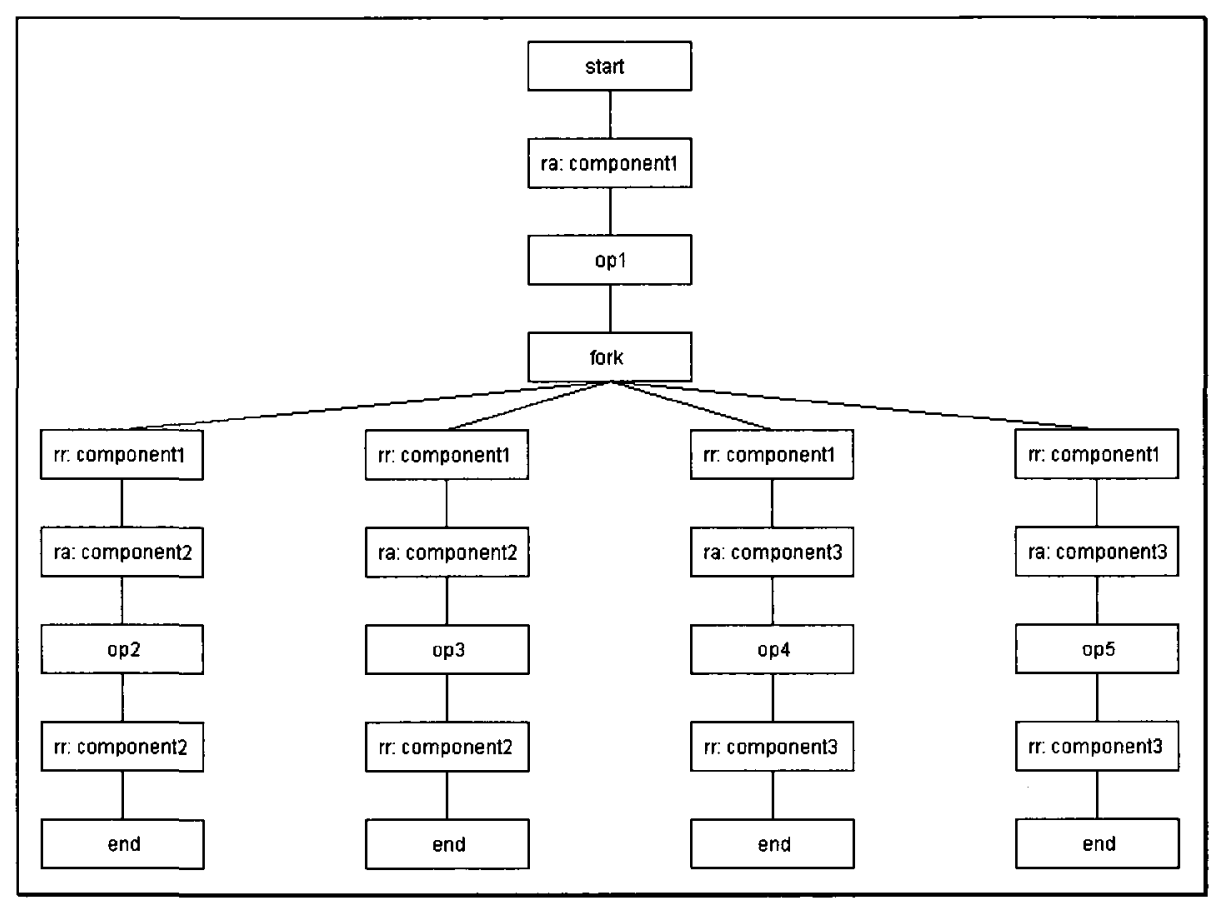

Figure 6-4: Test Case \#2 - Target Model

\subsubsection{Test Case \#3}

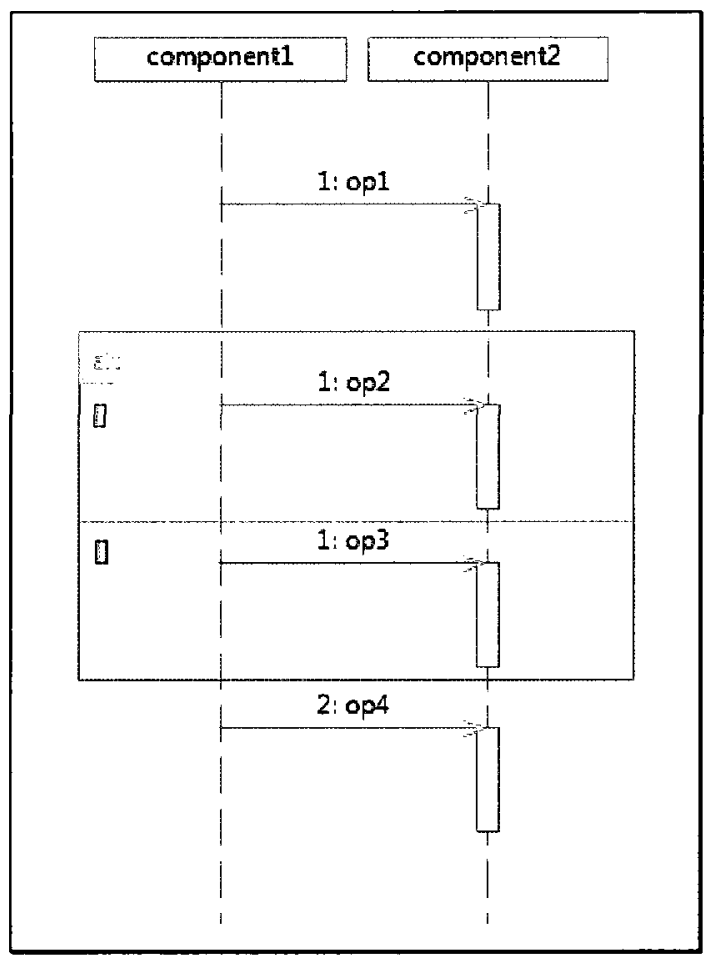

Figure 6-5: Test Case \#3 - Source Model 
This test case introduces the occurrence of fragments within UML sequence diagrams. In this case, the alt fragment is transformed in to Branch and Merge elements, both subclasses of PathConnections within the CSM. However in occurrences such as the one in Figure 3-5, these PathConnections may precede or succeed other PathConnections, such as the forks of asynchronous messages.

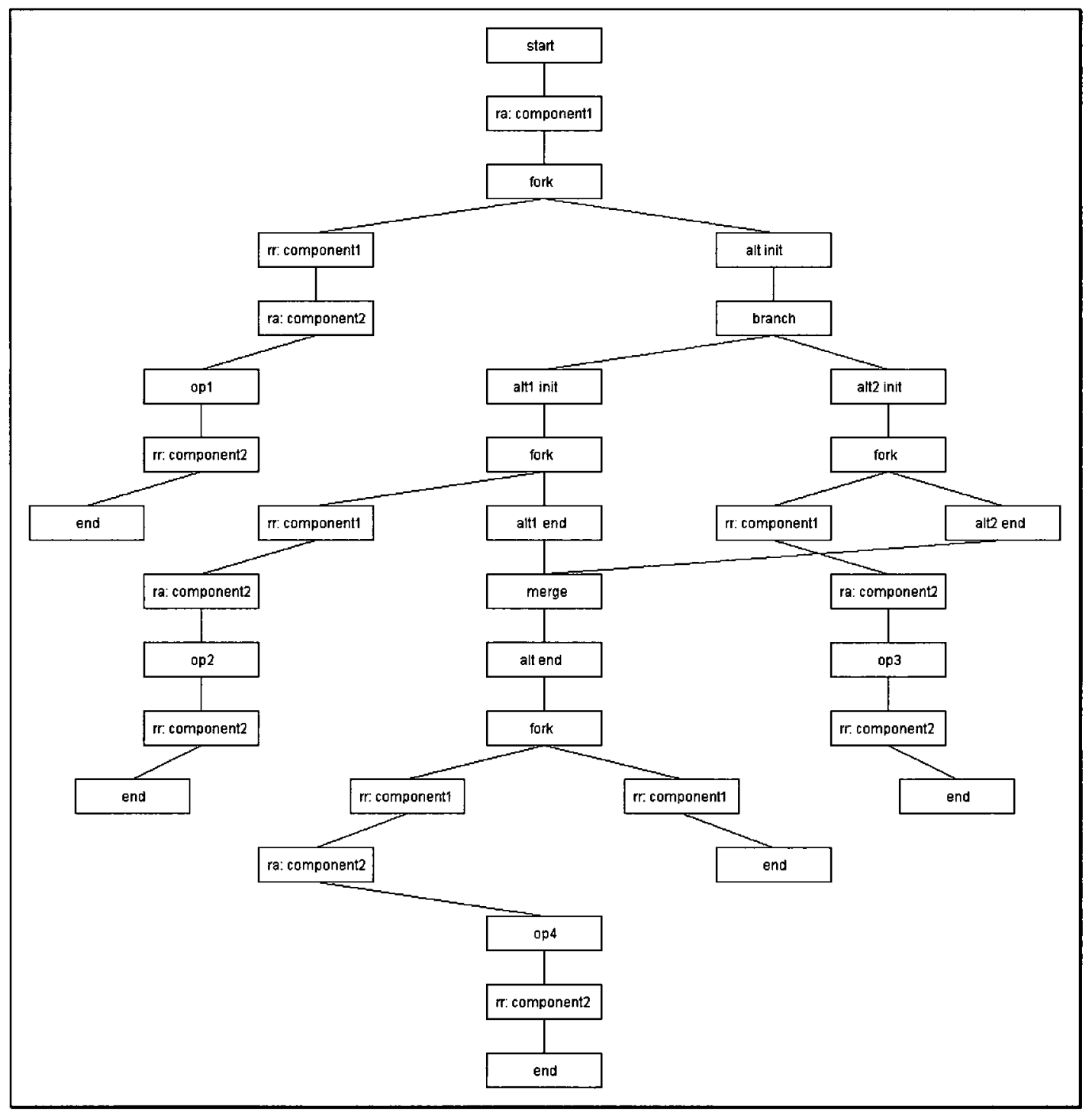

Figure 6-6: Test Case \#3 - Target Model 
In the target model (Figure 6-6), we can see a proper generation of dummy steps both within and outside the transformation elements of the fragment. Preceding the fragment is a lone asynchronous message with a fork, generating a dummy step "alt init" in the target model. Additionally, the operands in the source model, "alt1" and "alt2," each have asynchronous messages within them, therefore in the target model appears the "alt1 init" and "alt2 init" elements.

\subsubsection{Test Case \#4}

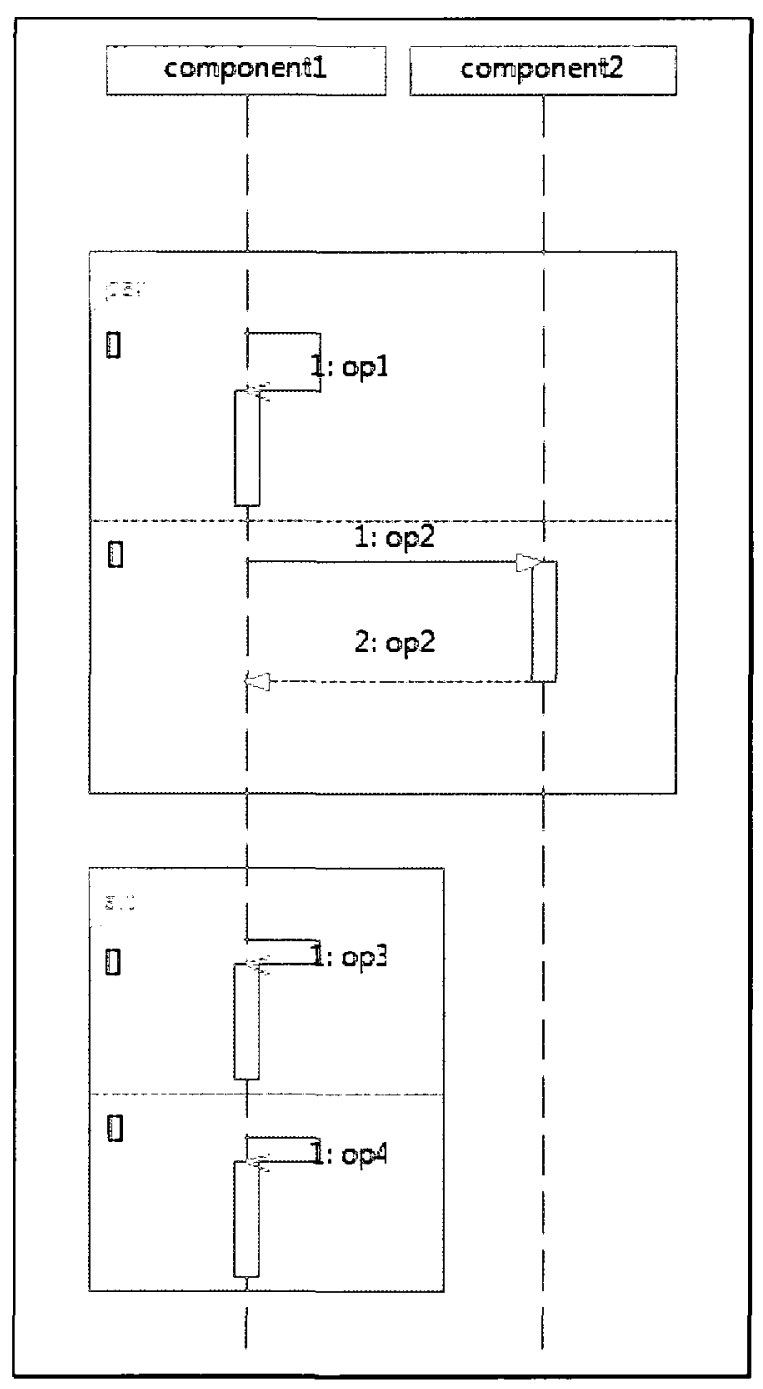

Figure 6-7: Test Case \#4 - Source Model 
This scenario tests the transformation of source models with fragments in sequence of each other. Another type of fragment, par, is introduced in this sequence diagram. The two PathConnections generated in the target model in this case are forks and joins. Succeeding the par fragment is an alt fragment, which as in the previous test case transforms in to a branch and merge in the target model. In the CSM, the branch succeeds the join, and since both are PathConnections, a dummy step must be inserted in between the two. Figure 6-8 illustrates the proper transformation of succeeding fragments.

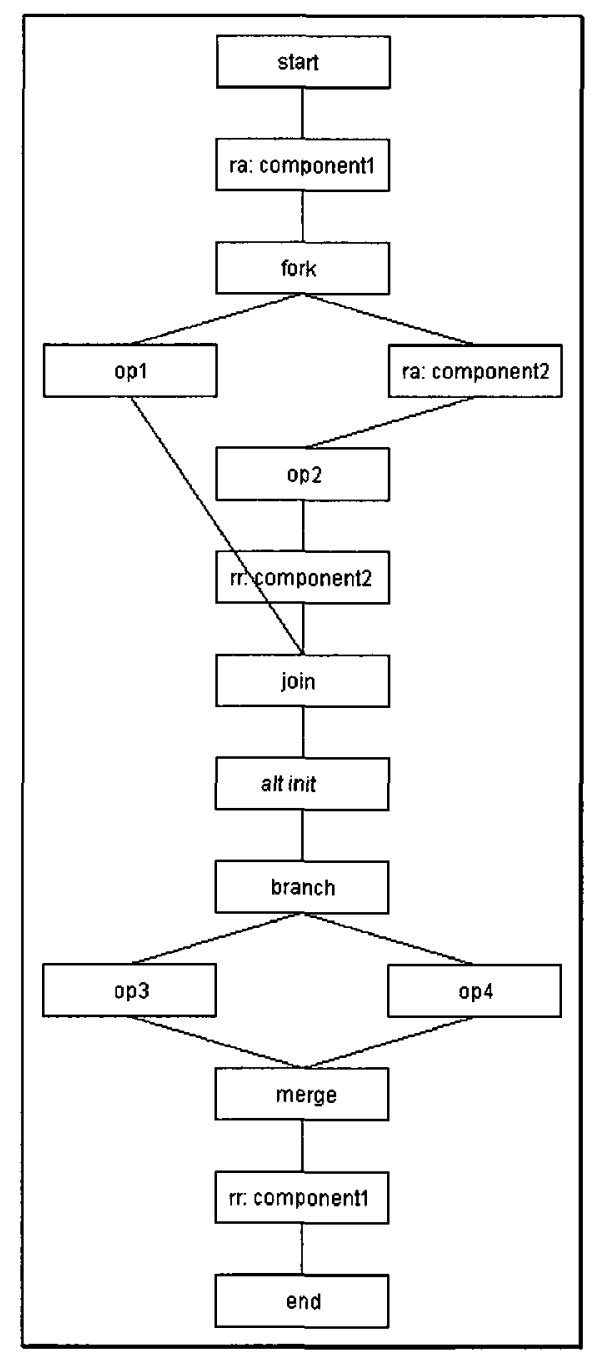

Figure 6-8: Test Case \#4 - Target Model 


\subsubsection{Test Case \#5}

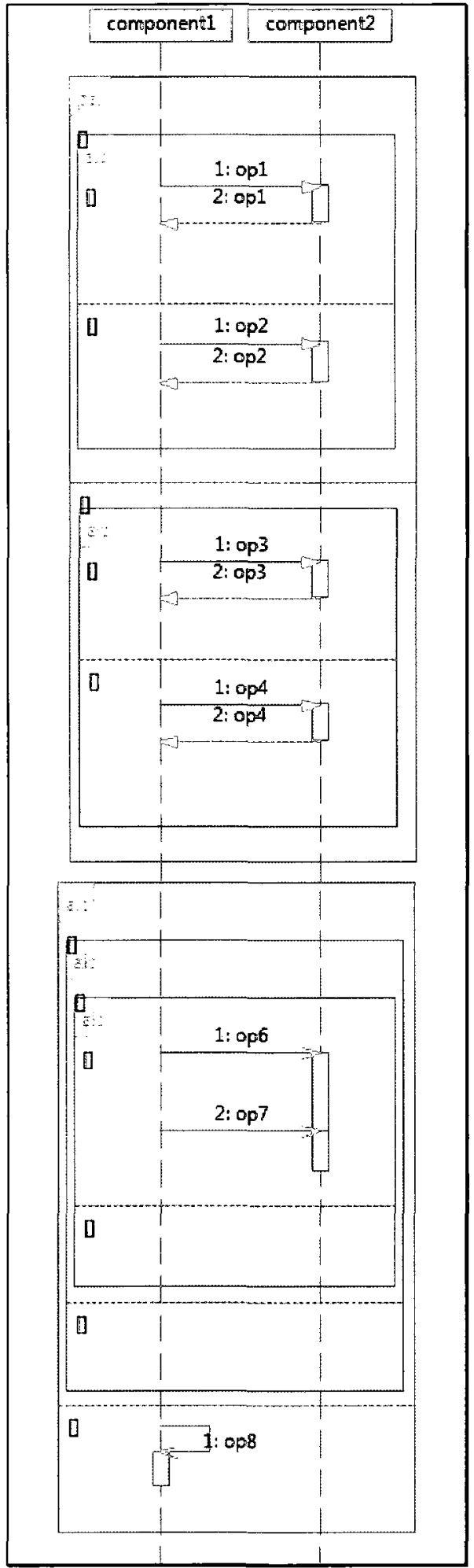

Figure 6-9: Test Case \#5 - Source Model 


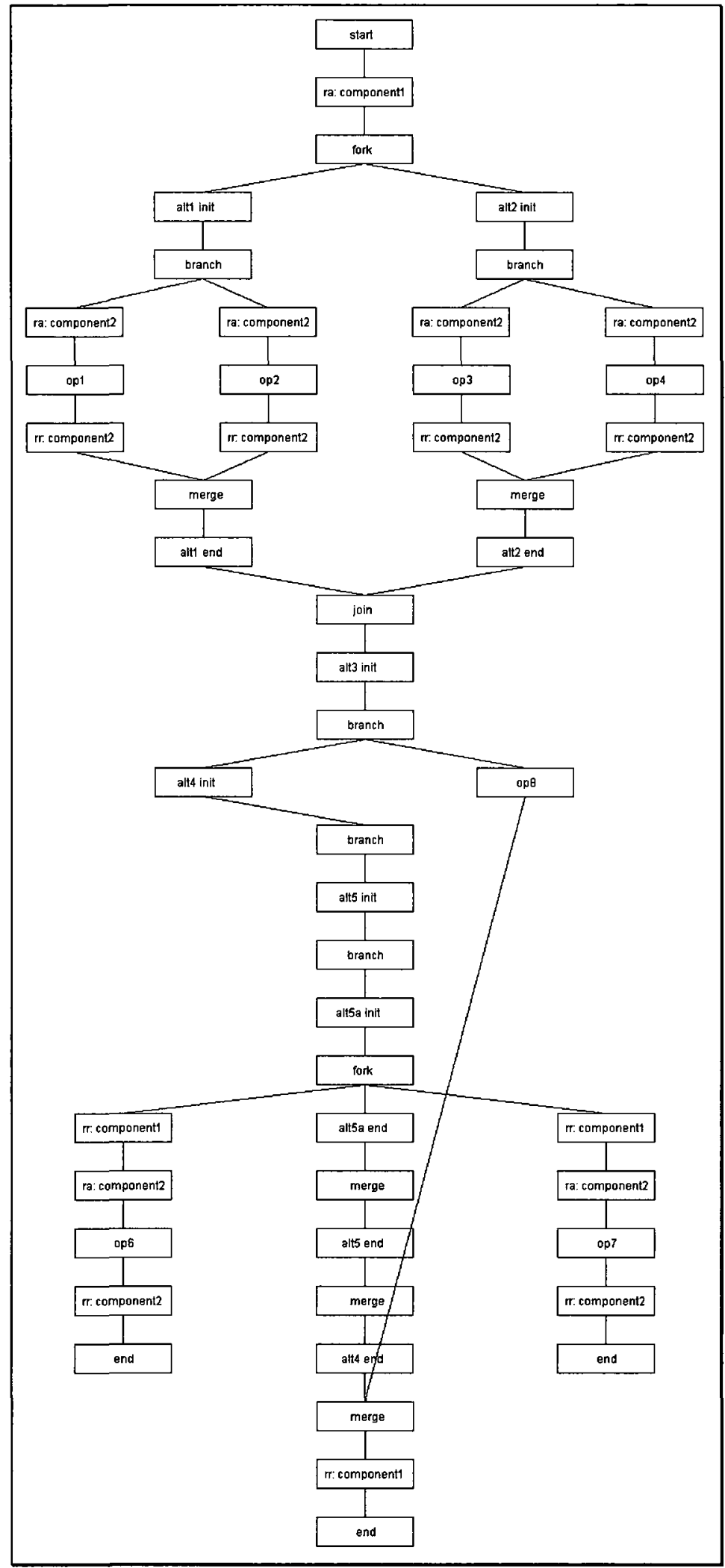

Figure 6-10: Test Case \#5 - Target Model 
This test case provides a much more complex scenario in which several fragments are nested within each other. Additionally, there are two fragments in sequence (just as in the previous test case). This type of source model transforms in to a fairly detailed CSM, where dummy steps have to be inserted in between the various generated PathConnections.

In the first fragment, which is a par, alt fragments are nested within each operand. This means that in the resultant target model, branches will appear after the initial fork (from the parent fragment). Also, merges precede the join (which is also from the parent fragment). A dummy step needs to be inserted in between each of these. Also, because a fragment is the successor, a dummy step appears after the join in the target model.

The second fragment consists of three layers of alt fragment nested within each other. The same logic as in the transformation of previous nested fragments follows here, with dummy steps appearing before and after each respective merge and branch. Also, because asynchronous messages "op6" and "op7" are within an alt fragment, the single fork message that is generated in the target model requires a dummy step between it and the branch that precedes it.

\subsubsection{Test Case \#6}

In this scenario, the transformation of loop fragments is tested. Loop fragments differ from alt and par fragments in that they don't transform in to any PathConnections, but rather in to single steps linked to sub-scenarios through refinements. Therefore in this case, sandwiched in between the generated branch and merge transformed from the alt fragment is a loop step linked to a sub-scenario (generated from the internal structure of the loop fragment). 


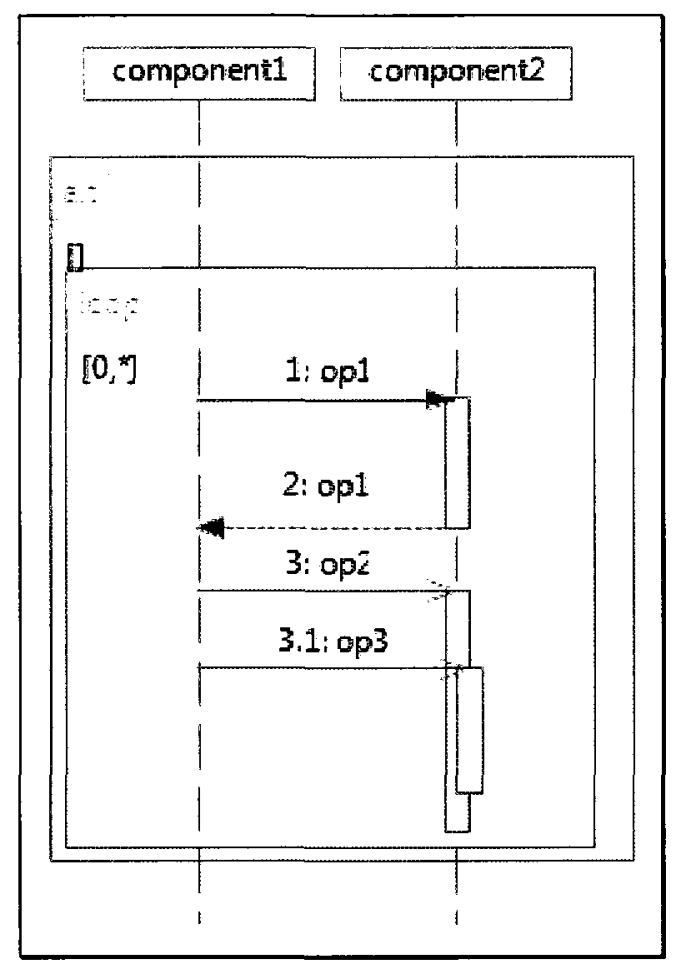

Figure 6-11: Test Case \#6 - Source Model

Since the loop fragment begins with a synchronous message, "op1," the sub-scenario in the target model contains the transformed elements of "opl" after the loop start. Succeeding the "op1" message in the source model are two asynchronous messages that are completely parallel within one another (they both have the same sender and receiver lifelines). This results in a fork and join within the sub-scenario in the target model. However, since there is no successor to "op3" in the source model, the fork is linked to the loop end, which always exists on the original sender lifeline of the loop fragment (in this case "component1"). 


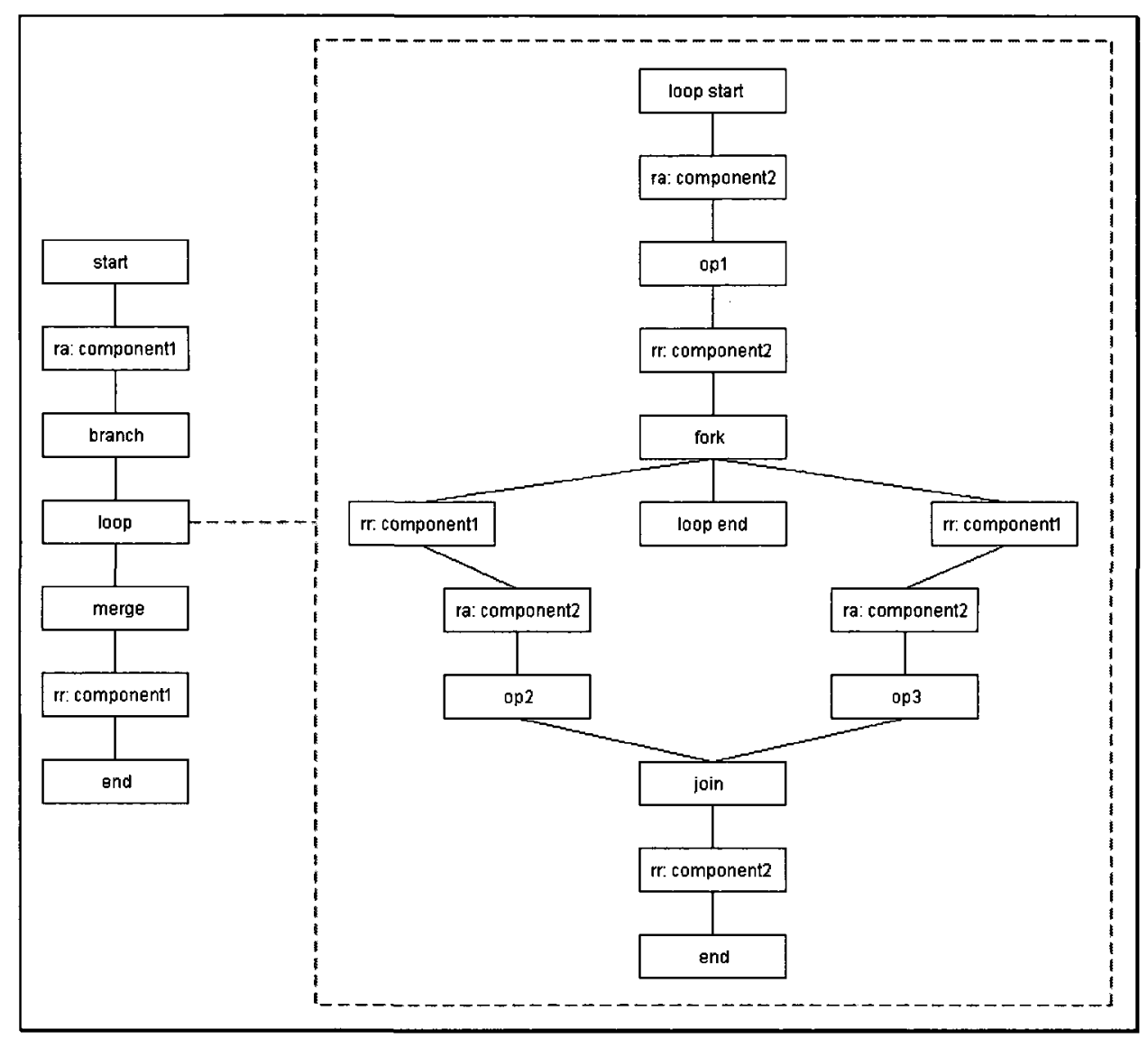

Figure 6-12: Test Case \#6 - Target Model

\subsubsection{PUMA Example Test Case}

This test case consists of a source model defined in Figure 4 of the PUMA paper [WPPSIM+05]. The resulting target model was nearly identical, but contained some minor differences due to the differences in transformation logic in this study and the paper's.

Within the second operand of the par fragment, is an asynchronous log message which gets transformed in to an additional fork in the target model (after the one generated from the par fragment). The transformation model in this study does not attempt to combine forks in these cases, due the algorithmic complexities involved. 
Other differences in the target models exist: such as the resource acquire and release steps that appear before and after the "readCache" step, respectively; and the end step that appears after the "log" target elements. Otherwise, the target models share the same structure.

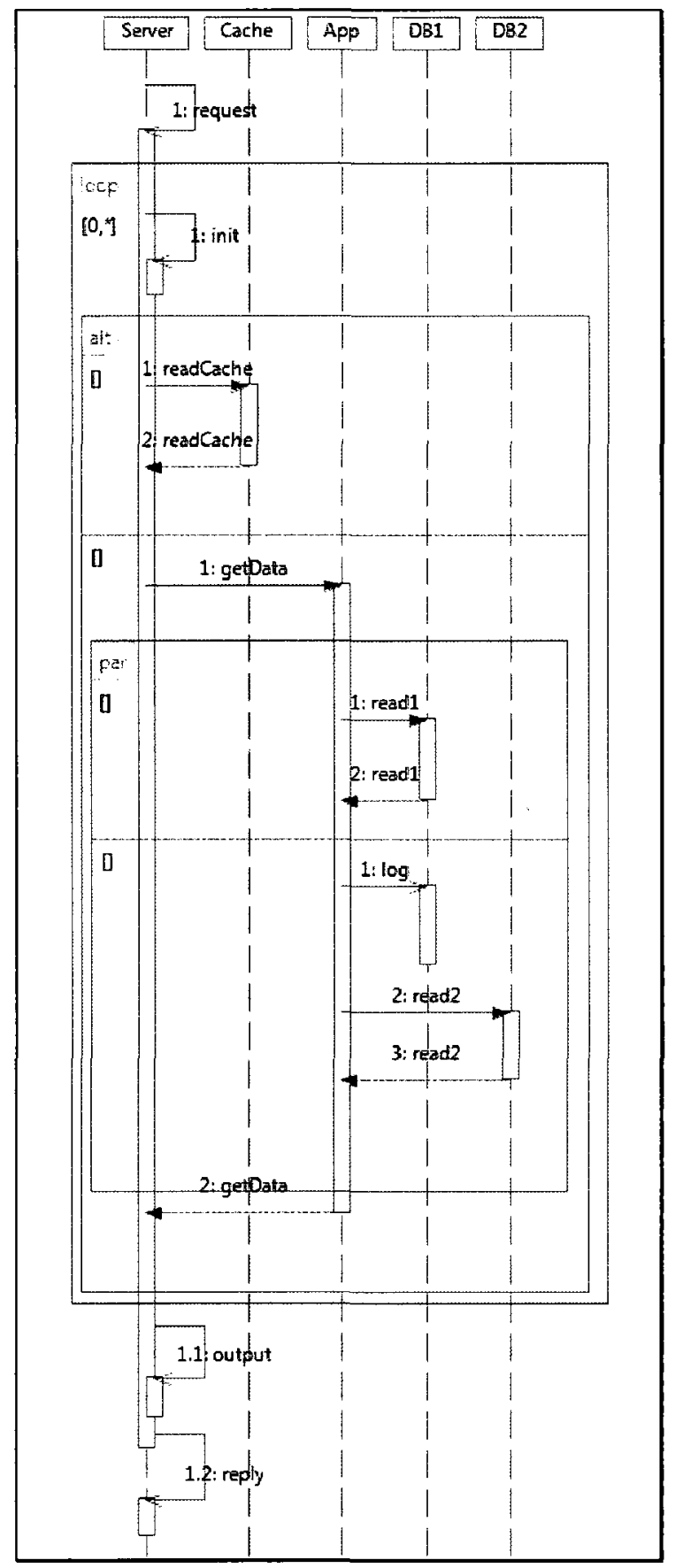

Figure 6-13: PUMA Example Test Case - Source Model 


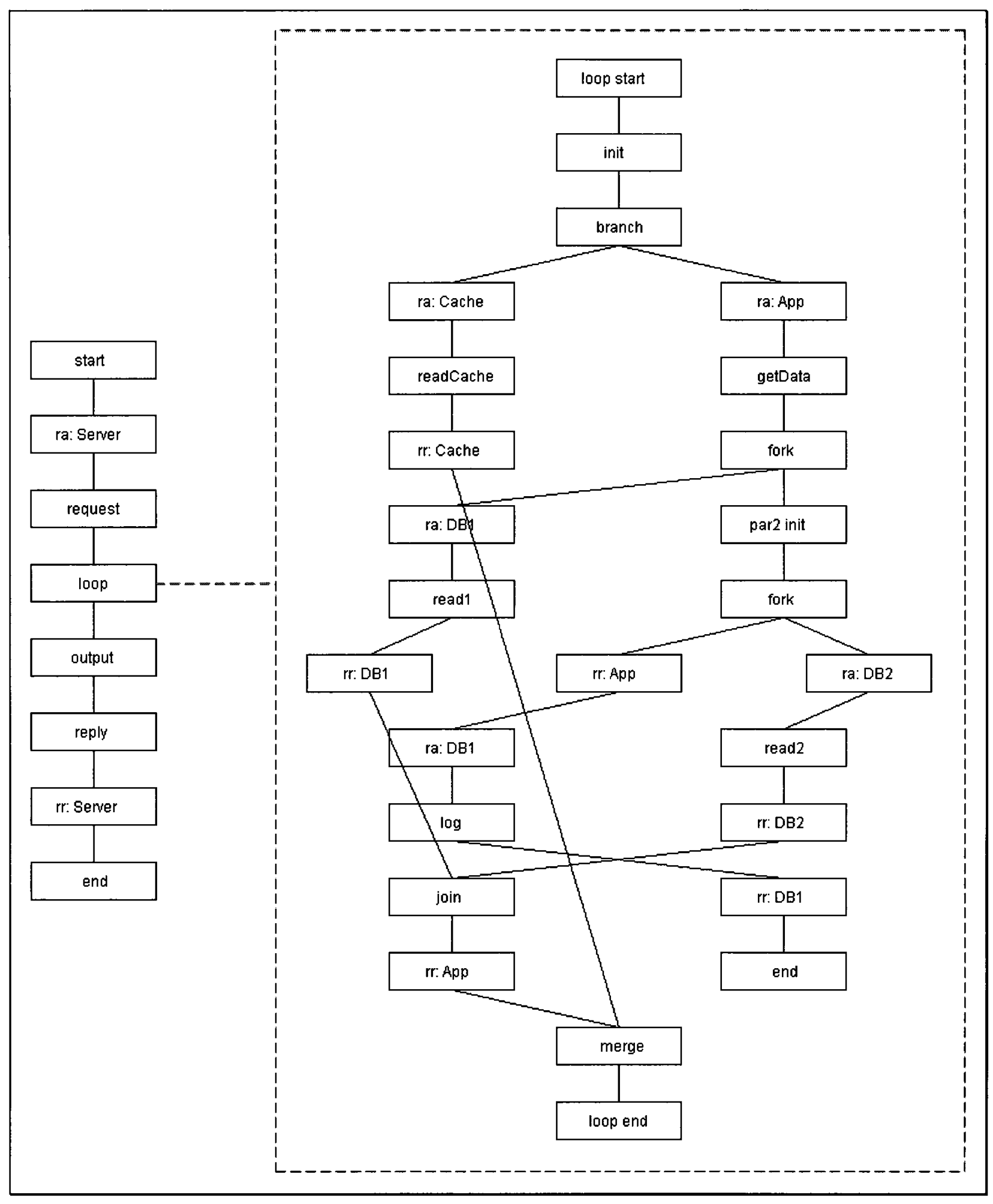

Figure 6-14: PUMA Example Test Case - Target Model 


\subsection{Case Study: Video Acquisition Scenario}

\subsubsection{Overview}

The case study involves the transformation of a scenario previously described in $[\mathrm{PW}+03],[\mathrm{XWP}+03]$. Other than the cycleInit operation, this entire scenario is embedded within a loop fragment that operates under a closed workload. Each lifeline has an applied MARTE stereotype, whether a PaLogicalResource in the case of physical resources (such as the Video Controller, BufferManager and Database), or PaRunTInstance in the case of software process (such as GetImage and StoreProc). Messages also have applied stereotypes, in the form of PaStep, which have a hostDemand attribute specifying the mean processor runtime of the operation.

\subsubsection{Source Model Creation}

The source model is defined in RSA by creating a new MARTE Model within an existing project. Then within the MARTE Model, a UML Sequence Diagram can be created. The diagram elements can constructed by simple drag and drop gestures.

While a sequence diagram element is highlighted, its properties can be edited in the bottom frame of Figure 6-15. It's also here that the MARTE stereotypes can also be applied to each element, as well as have their attribute values set. Although RSA doesn't display the stereotype details on the diagram, the Note tool on the right-hand pane can be used to drop textual descriptions in to the diagram. 


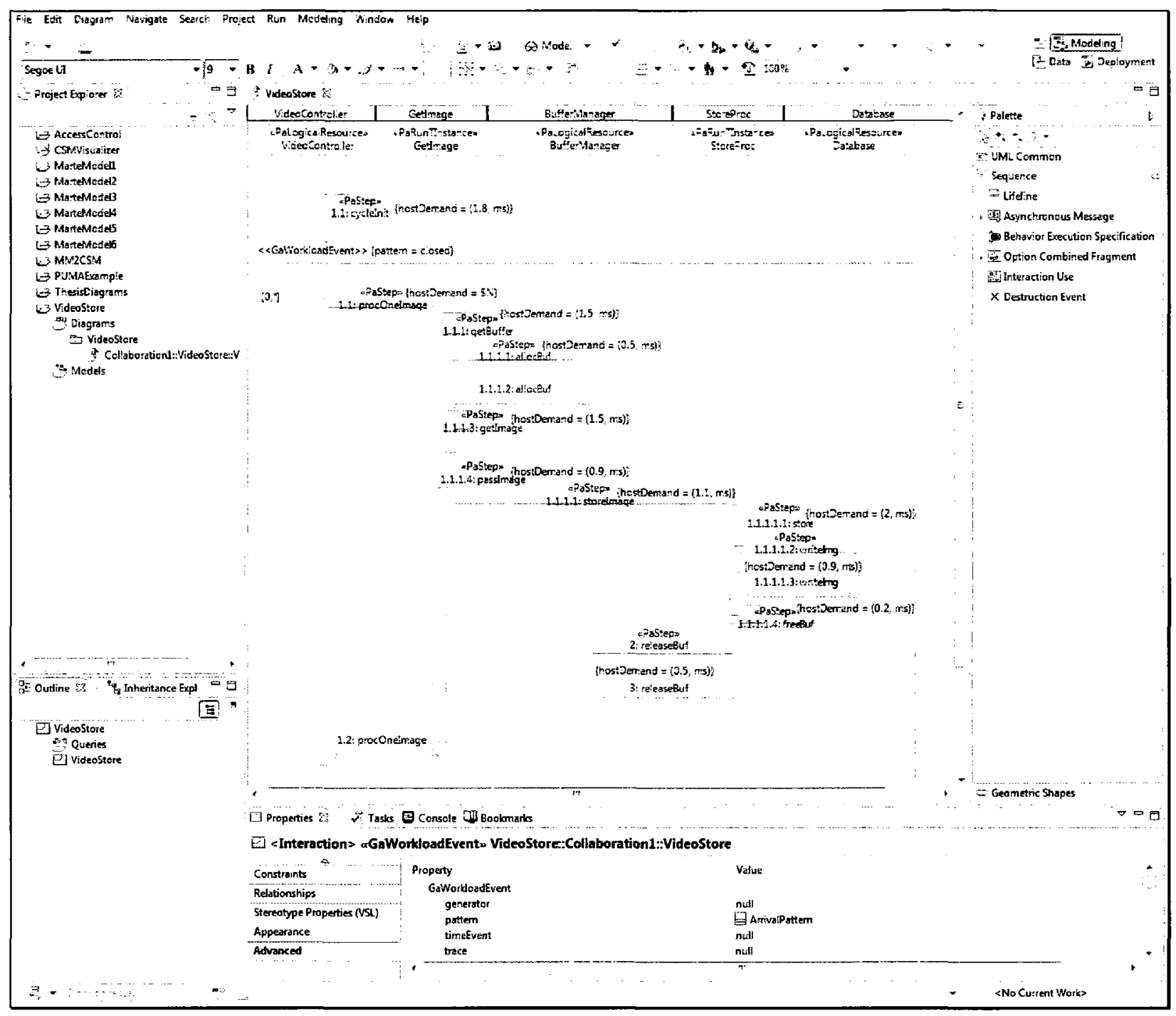

Figure 6-15: RSA Modeling View

\subsubsection{Source Model XMI Export}

After the source model has been fully constructed under RSA, the next step is to export the model in XMI form so that it can be read by the ATL transformation. Under the file menu, is an item called Export. Accessing this brings up a dialog screen (Figure 6-16) that provides the user with an option for selecting the format to export the UML model. In this case, UML 2.1 Model is the desired option. 


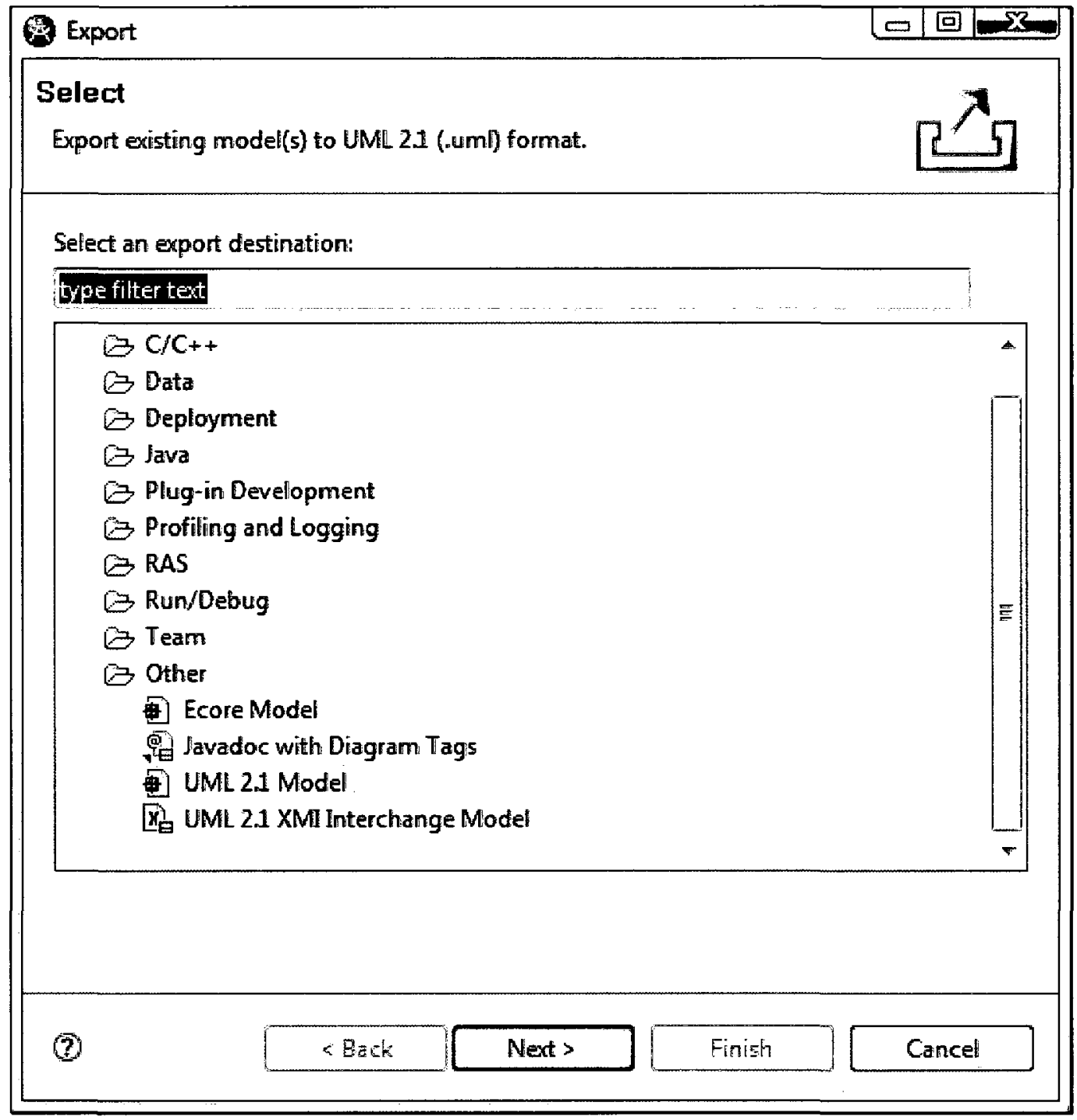

Figure 6-16: Source Model XMI Export Dialog - $1^{\text {st }}$ Screen

After clicking next, a second dialog screen prompts the user to select the model for export and the destination of the exported model. Simply browse to VideoStore.emx for the source, and select the input folder of the ATL transformation model project (/MM2CSM/Input) as the target. Also, it's important to select both checkboxes, as in Figure 6-17, to make sure all necessary UML profiles are exported. Should the dialog ask whether to rewrite a file, it's best to approve the option for everything. 


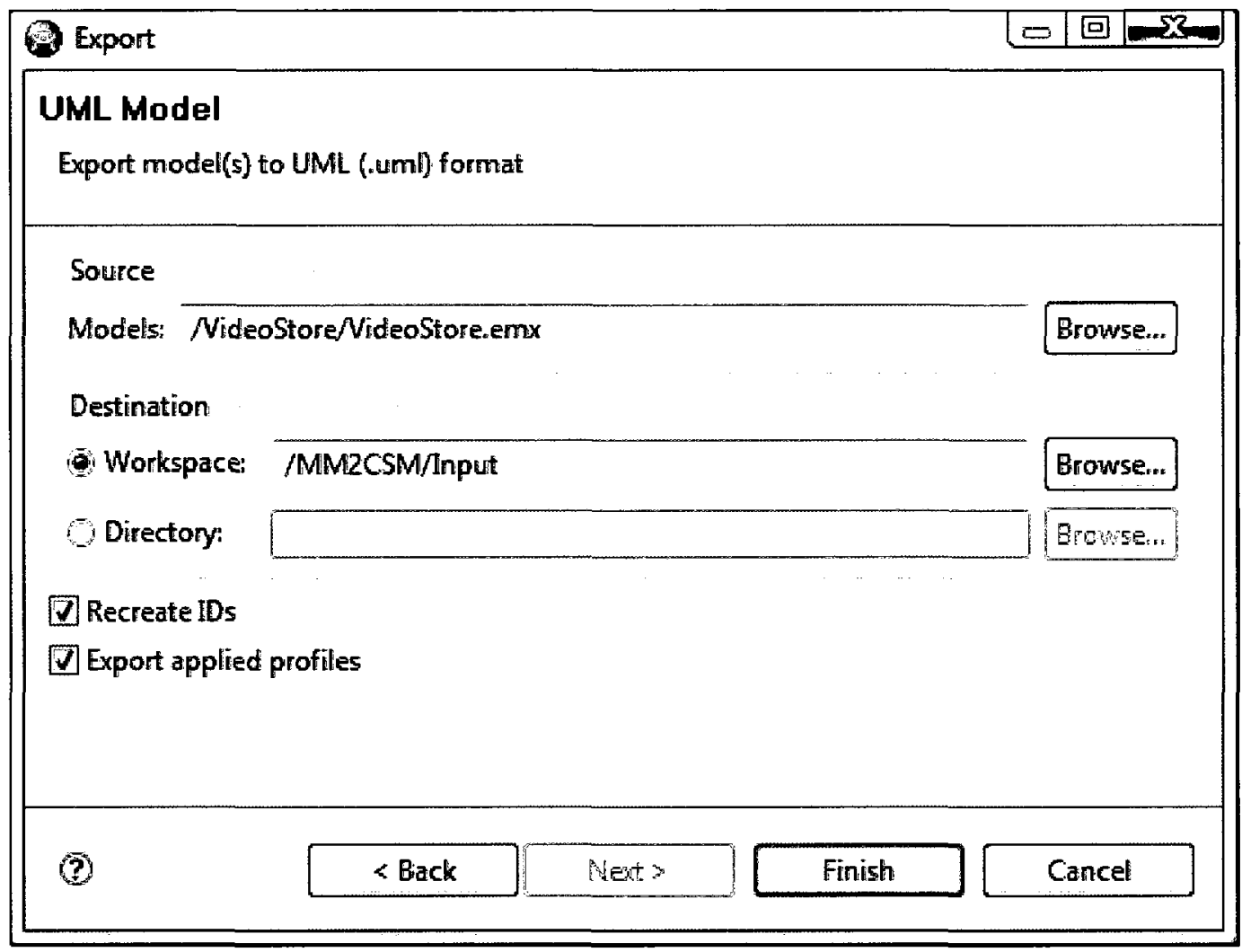

Figure 6-17: Source Model XMI Export Dialog - $2^{\text {nd }}$ Screen

A file by the name VideoStore.uml should now reside in the input folder of the MM2CSM project.

\subsubsection{Run Configuration}

Running a ATL transformation requires creating a run configuration within Eclipse. Doing so involves accessing the Run Configurations item under the Run menu. In the dialog that appears, creating a new ATL transformation run configuration presents several fields for specifying the configuration name, project, ATL module, source and target metamodels, the source model, target model destination, as well as any libraries in use. Filling out these fields, as in Figure 6-18, creates the desired ATL transformation run configuration. 


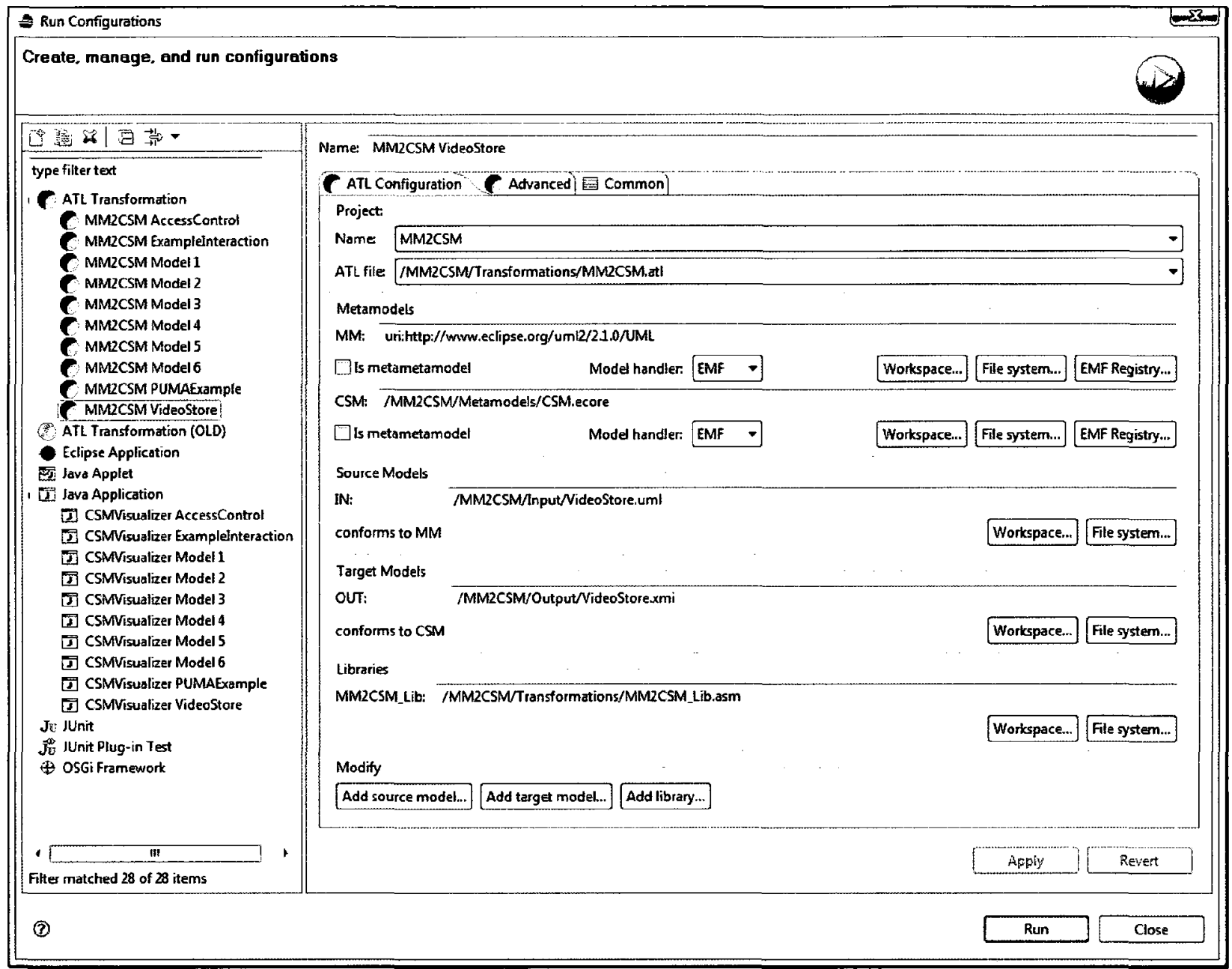

Figure 6-18: ATL Transformation Run Configuration

While in the Run Configurations dialog, it is also useful to create a run configuration for the CSM Visualizer. This is done by creating a new run configuration under the Java Application menu. Setting the Project and Main Class fields (shown in Figure 6-19) below creates a runnable instance of CSM Visualizer. However it is still required to specify the location of the target model in the Program Arguments field within the Arguments tab in order to create a visualization of the correct target model. 


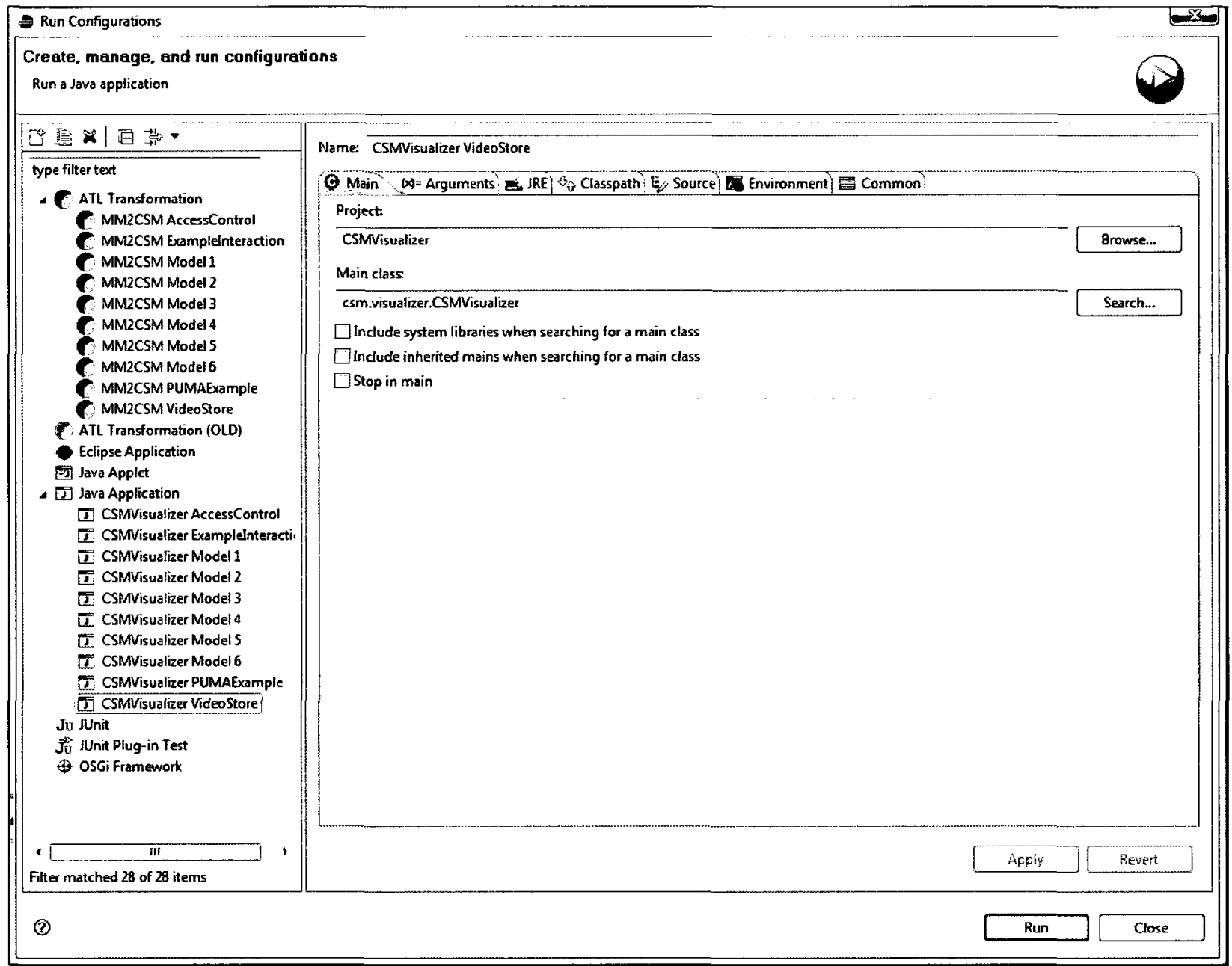

Figure 6-19: CSM Visualizer Run Configuration

\subsubsection{Transformation Execution}

In the Run Configurations dialog screen, selecting on "MM2CSM VideoStore" and hitting run executes the ATL transformation, MM2CSM, on the VideoStore.uml source model. A new file, VideoStore.xmi, should now reside in the output folder of the MM2CSM project (/MM2CSM/Output). 


\subsubsection{CSM Visualizer Execution}

In the Run Configurations dialog screen, selecting "CSMVisualizer VideoStore" and hitting run executes an instance of the CSM Visualizer with VideoStore.xmi as the source. As can be seen in Figure 6-20, a frame on the left provides the selection of one of the scenarios in the CSM. Whichever scenario is selected is visualized in the right-hand pane. Although the CSM Visualizer does not show the transformation of the MARTE applied stereotypes, an inspection of the XMI target model of the Video Store (see Appendix) verifies the proper transformation of these elements.

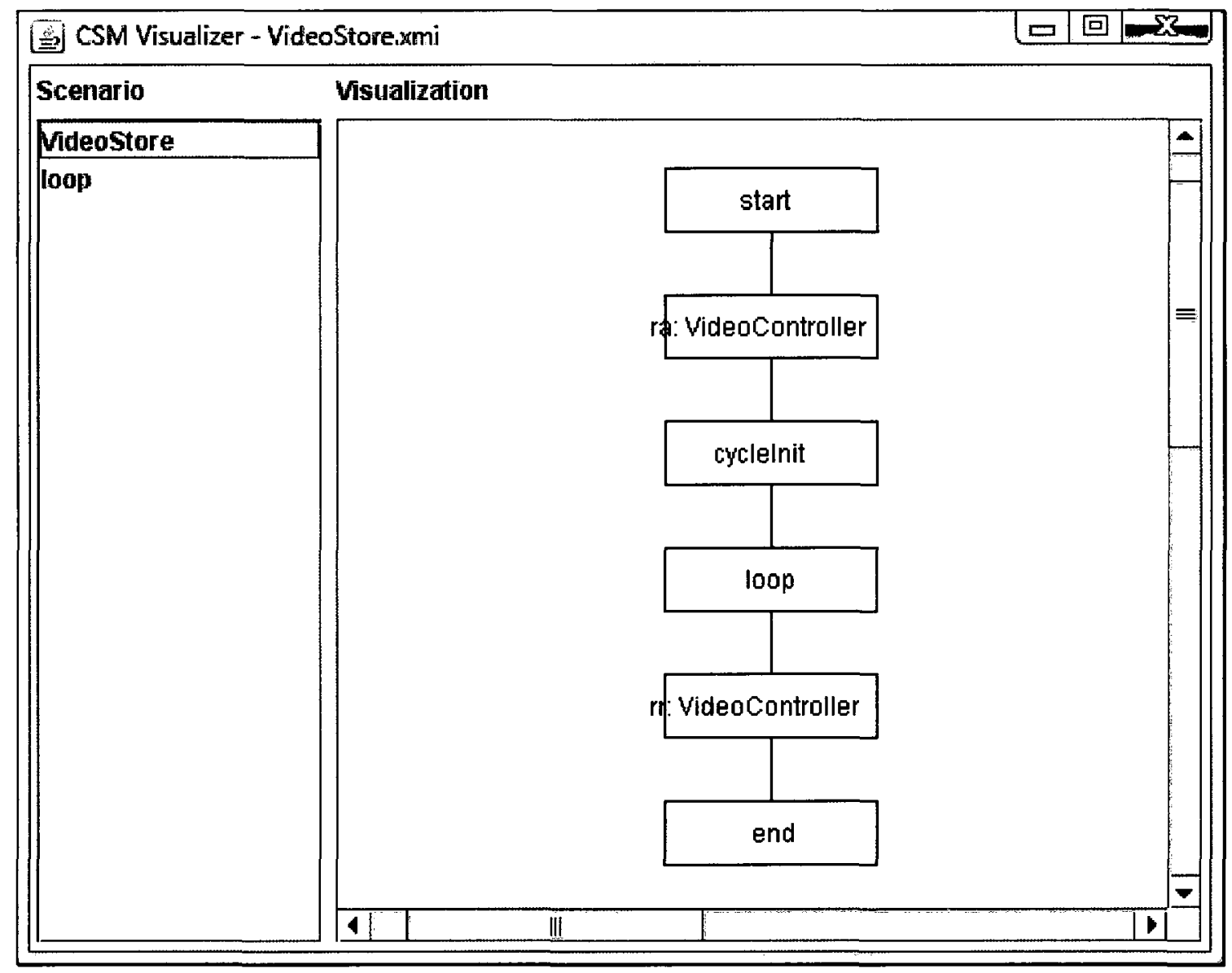

Figure 6-20: CSM Visualizer Execution 


\section{Chapter 7. Conclusions}

\subsection{Accomplishments}

This thesis proposed and implemented a rule-based model transformation in a specialized transformation language, ATL, which transforms UML 2.1 sequence diagrams (with applied MARTE stereotypes) in to Core Scenario Models. The Eclipse environment accepted XMI files exported from the RSA UML modeling tool and virtualized the model internally using its UML EMF library. The ATL engine was then able to accept this virtual model as the source model and execute the transformation module, MM2CSM, which also accepted the custom ECORE written CSM metamodel. Using the CSM metamodel, ATL was able to produce an XMI file of the target model, which could then be read by the CSM Visualizer to produce a CSM diagram. This combined process satisfied the chief objective laid out in Chapter 1 of implementing an ATL transformation based on the concepts laid out in the PUMA paper [WPPSIM+05]. The length of the ATL transformation project is about 3400 lines of code (2300 for the MM2CSM module and 1100 for the MM2CSM_Lib module). Within the MM2CSM module exists 12 matched rules, to handle unconditional mappings, and 30 lazy rules, to handle conditional mappings. The MM2CSM_Lib module contains 94 helpers that are be invoked by the transformation rules defined in MM2CSM.

Also achieved in this thesis was the successful development of platform independent transformation mappings of the domain problem that can be used as a base design in implementations of other rule-based languages. Similarly, another significant accomplishment is the development of design methodologies that can be used in other domain problems with rule- 
based languages and cases specific to ATL. This work complements some of the proposed patterns in other works, like in the "Reusable Model Transformation Patterns" paper [ISH+08].

Last, but not least of the accomplishments of this thesis was the development of the CSM Visualizer, which allowed for human visual verification of CSMs. The algorithm used in constructing each CSM diagram may prove useful in other applications where serialized models need to be visually constructed. The total length of the Java code of the CSM Visualizer is a little over 1000 lines.

\section{$\underline{7.2 \text { Limitations }}$}

There are a few limitations with the ATL model transformation of this thesis. Chief among them were the restrictions of the source models created in RSA.

As previously stated, the transformation can accept only UML sequence diagrams that have a single starting message. Every message other than the first is caused by some preceding message on the sender lifeline. The reasons for this limitation lie more within the time constraints of this thesis, since multiple starting points in UML sequence diagrams would cause a dramatic increase in the number of mapping rules. This would make implementing the large number of transforming rules in ATL exceedingly difficult and time consuming.

The same reasoning applies behind the operand limitation within fragments. The first message in each operand must have a common sender lifeline. Additionally, the containing fragment cannot be a starting point in the sequence diagram, unless it is the very first event in the model. Violating this and the previous guideline has unpredictable consequences in the resultant target model, including many cases where the ATL execution engine crashes midway during a transformation. 
Another limitation of the work in this thesis was due to the incomplete development of the third party MARTE plugin for RSA. The plugin was unable to link the source model to elements annotated with MARTE stereotypes in UML deployment diagrams. As a result of this restriction, source models did not have all the required elements to allow for transformation mappings of more detailed Resource elements in the target models. For instance, PaRunTInstance and PaLogicalResource stereotypes will only produce target model Components in active and passive states, respectively, but all the rest of the Component attributes will be left blank.

\subsection{Future Work}

Although the transformation process for UML+MARTE sequence diagrams to CSMs was successfully realized in this thesis, there are several opportunities for future work:

- Increased MARTE transformation capability - As previously stated, the limitations of the MARTE plugin tool for RSA hindered the ability to develop complete transformation functionality of UML+MARTE models to CSMs. An updated plugin would allow for more detailed transformation capabilities, especially with regards to Resources in the target model.

- Bidirectional Transformations - This would allow the backtracking of CSMs to UML+MARTE models, although several challenges remain in implementing this. The PUMA paper [WPPSIM+05] did not provide any specifications for backtracking, and a domain problem analysis could prove difficult as CSMs are abstractions of UML models, meaning the inverse process transformation will be complex. 
- Expanded CSM Visualizer Scope - The current utility only displays a graph of the Steps and PathConnections that make up a Scenario in a CSM. The scope of the CSM Visualizer could be increased by adding visual elements for Workflows, Refinements and Resources and their relationships to existing elements in CSM Diagrams. However, this expanded scope would require more CSM specifications beyond those laid out in the PUMA paper [WPPSIM+05].

- Transformation Integration - As demonstrated throughout the course of this study, the entire transformation process takes place among different platforms, requiring many manual user steps. For reasons of transparency and convenience, it would be extremely beneficial if all these steps (including running the CSM Visualizer) could be integrated seamlessly. This way, creating transformation instances and executing them would become more user-friendly, as they would require fewer steps from the user. 


\section{References}

[ATL]

[BJP+05]

$[\mathrm{BM}+08]$

[CGM+08]

$[\mathrm{CH}+03]$

[Eclipse]

$[\mathrm{FHL}+06]$

[GHJV+95]

[ISH+08]

[Java]

$[\mathrm{JA}+06]$
ATLAS Group. Atlas Transformation Language. 2009. http://www.eclipse.org $/ \mathrm{m} 2 \mathrm{~m} / \mathrm{atl} /$.

Jean Bézivin, Frédéric Jouault, Jean Paliès. Towards Model Transformation Design Patterns. ATLAS Group (INRIA \& LINA, University of Nantes), 2005.

Alexandre Bragança, Ricardo J. Machado. "Transformation Patterns for Multi-staged Model Driven Software Development." Proceedings of the 2008 12th International Software Product Line Conference. Washington, DC, USA: IEEE Computer Society, 2008. 329-338.

Vittorio Cortellessa, Sara Di Gregorio, Antinisca Di Marco. "Using ATL for transformations in software performance engineering: a step ahead of javabased transformations?" Proceedings of the 7th international workshop on Software and performance. Princeton, NJ, USA: ACM, 2008. 127-132.

Krzysztof Czarnecki, Simon Helson. "Classification of Model Transformation Approaches." OOPSLA'03 Workshop on Generative Techniques in the Context of Model-Driven Architecture, University of Waterloo, 2003.

The Eclipse Foundation. Eclipse IDE 3.4. 2009. http://www.eclipse.org/.

Yong-Yi Fanjiang, Nien-Lin Hsueh, Jonathan Lee. "A Pattern-based Model Transformation Approach to Enhance Design Quality." JCIS-2006 Proceedings. Atlantis Press (October 2006).

Gamma, E., Helm, R., Johnson, R. and Vlissides, J. (1995). Design patterns Elements of Reusable Object-Oriented Software, Addison-Wesley.

Maria-Eugenia Iacob, Maarten W.A. Steen, Lex Heerink. "Reusable Model Transformation Patterns." Enterprise Distributed Object Computing Conference Workshops. Munich, Germany: IEEE, 2008. 1-10.

Sun Microsystems. Java Platform Standard Edition. 2009. http://java.sun.com/javase/index.jsp.

Frédéric Jouault, Freddy Allilaire. "The ATL Virtual Machine - An introduction to the ATL Virtual Machine V1.0 draft." ATLAS group (INRIA \& LINA), University of Nantes, France. 2006. http://www.sciences.univnantes.fr/lina/atl/. 
[KBJ+06] Ivan Kurtev, Klaas van den Berg, Frédéric Jouault. "Evaluation of Rule-based Modularization in Model Transformation Languages illustrated with ATL." Proceedings of the 2006 ACM Symposium on Applied Computing. Dijon, France: ACM, 2006. 1202 - 1209.

[KFJ+07] Jacques Klein, Franck Fleurey, Jean-Marc Jézéquel. "Weaving Multiple Aspects in Sequence Diagrams." Lecture Notes in Computer Science (Springer-Verlag) Vol. 4620 (2007): 167-199.

[MARTE] Object Management Group. "A UML Profile for MARTE: Modeling and Analysis of Real-Time Embedded systems, Beta 2 - ptc/2008-06-09." 2008. http://www.omgmarte.org/.

[MARTEPlugin] Sebastien Demathieu, Laurent Rioux. MARTE Profile for Rational Software Architect (RSA) 7.0. 2007. http://www.omgmarte.org/Tools.htm

[MDA]

[Miller+04] Sheena Judson Miller. "Pattern-Based Model Transformation: A Metamodelbased Approach to Model Evolution." Dissertation, Department of Computer Science, Louisiana State University, 2004

$[\mathrm{MOF}]$

Object Management Group. "Meta Object Facility (MOF) Core Specification OMG Available Specification Version 2.0 - formal/06-01-01." 2006. http://www.omg.org/technology/documents/modeling spec catalog.htm.

[OCL] Object Management Group. "Object Constraint Language OMG Available Specification Version 2.0 - formal/06-05-01." 2006. http://www.omg.org/technology/documents/modeling spec catalog.htm.

[PW+03] Dorina C. Petriu, C.M. Woodside,"Performance Analysis with UML", chapter in UML for Real: Design of Embedded Real-Time Systems (L. Lavagno, G. Martin and B.Selic, Eds.), pp. 221-240, ISBN 1-4020-7501-4, Kluwer Academic Publishers, 2003.

[PW+04] Dorin B. Petriu, Murray Woodside. "A Metamodel for Generating Performance Models from UML Designs." Lecture Notes in Computer Science (Springer-Verlag) Vol. 3273 (2004): 41-53.

[PW+06] Dorin B. Petriu, Murray Woodside. "An intermediate metamodel with scenarios and resources for generating performance models from UML designs." Software and Systems Modeling (Springer-Verlag), 2006: 163-184. 
[QVT]

[RSA]

[SLSS+08]

[SoaML]

[SPT]

$[\mathrm{SV}+06]$

[SysML]

[UML2Infra]

[UML2Super]

[Varró+06]

[WPPSIM+05]

[Xerces]
Object Management Group. "Meta Object Facility (MOF) 2.0 Query/View/Transformation Specification - formal/2008-04-03." 2008. http://www.omg.org/technology/documents/modeling spec catalog.htm.

IBM. Rational Software Architect 7.5 Standard Edition. 2008. http://www-01.ibm.com/software/awdtools/swarchitect/standard/.

Mika Siikarla, Markku Laitkorpi, Petri Selonen, Tarja Systä. "Transformations Have to be Developed ReST Assured." Lecture Notes In Computer Science (Springer-Verlag) Vol. 5063 (2008): 1-15.

Object Management Group. "Service oriented architecture Modeling Language (SoaML) - ptc/2009-04-01." 2009.

http://www.omg.org/technology/documents/modeling spec catalog.htm.

Object Management Group. "UML ${ }^{\mathrm{TM}}$ Profile for Schedulability, Performance, and Time Specification - formal/05-01-02." 2005. http://www.omg.org/technology/documents/formal/schedulability.htm

Thomas Stahl, Markus Völter. Model-Driven Software Development. John Wiley \& Sons, Ltd. 2006. ISBN 0-470-02570-0.

Object Management Group. "OMG SysML Specification - ptc/06-05-04." 2006.

http://www.omg.org/technology/documents/modeling spec catalog.htm.

Object Management Group. "Unified Modeling Language: Infrastructure version 2.1.1 - formal/07-02-06." 2007.

http://www.omg.org/technology/documents/formal/uml.htm.

Object Management Group. "Unified Modeling Language: Superstructure version 2.1.1 - formal/2007-02-05." 2007.

http://www.omg.org/technology/documents/formal/uml.htm.

Varró, Dániel. "Model Transformation by Example." Lecture Notes in Computer Science (Springer-Verlag) Vol. 4199 (2006): 410-424.

Murray Woodside, Dorina C. Petriu, Dorin B. Petriu, Hui Shen, Toqeer Israr, Jose Merseguer. "Methods and Tools for Performance by Unified Model Analysis (PUMA)." Proceedings of the 5th international workshop on Software and performance. Palma, Illes Balears, Spain: ACM, 2005. 1-12.

The Apache Software Foundation. Apache Xerces Project. 2009. http://xerces.apache.org/. 
[XWP+03] Xu, J., Woodside, M., Petriu D. "Performance analysis of a software design using the UML profile for schedulability, performance and time."

Proceedings of the 13th International Conference on Computer Performance Evaluation, Urbana, Illinois, USA: Springer 2003. 291-310

[XMI] Object Management Group. "MOF 2.0/XMI Mapping, Version 2.1.1 - ptc/0710-06." 2007.

http://www.omg.org/technology/documents/modeling_spec catalog.htm. 


\section{Appendix: CSM XMI Output}

\section{A.1 Example Interaction}

$<$ ? $\underline{x m l}$ version="1.0" encoding="ISO-8859-1"? >

<Model xmi:version="2.0" xmlns:xmi="http://www.omg.org/XMI" xmlns:xsi="http://www.w3.org/2001/XMLSchema-

instance" xmlns="platform:/resource/MM2CSM/Metamodels/CSM.ecore" name="ExampleInteraction" >

<scenario name="interaction">

<step xsi:type="Start" name="start" successor="//@scenario.0/@pathConnection.0" workload="//@workload.0"/>

<step xsi:type="ResourceAcquire" name="ra: componentA" predecessor="//@scenario.0/@pathConnection.0"

successor="//@scenario.0/@pathConnection.6" acquire="//@resource.0"/>

<step name="op3" predecessor="//@scenario.0/@pathConnection.4"

successor="//@scenario.0/@pathConnection.5">

$<$ probability $>0.75</$ probability $>$

$<$ repCount $>5</$ repCount $>$

$<$ hostDemand $>(0.85, \mathrm{~ms})</$ hostDemand $>$

$</$ step $>$

<step xsi:type="ResourceAcquire" name="ra: componentB" predecessor="//@scenario.0/@pathConnection.1"

successor="//@scenario.0/@pathConnection.2" acquire="//@resource.1"/>

<step name="op1" predecessor="//@scenario.0/@pathConnection.2"

successor="//@scenario.0/@pathConnection.8" >

$<$ probability $>0.50</$ probability $>$

$<$ repCount $>3</$ repCount $>$

$<$ hostDemand $>(1.75, \mathrm{~ms})</$ hostDemand $>$

$</$ step $>$

<step xsi:type="ResourceRelease" name="rr: componentA" predecessor="//@scenario.0/@pathConnection.6" successor="//@scenario.0/@pathConnection.1" release="//@resource.0"/>

<step xsi:type="ResourceAcquire" name="ra: componentB" predecessor="//@scenario.0/@pathConnection.4"

successor="//@scenario.0/@pathConnection.3" acquire="//@resource.1"/>

<step name="op2" predecessor="//@scenario.0/@pathConnection.3"

successor="//@scenario.0/@pathConnection.7">

$<$ probability $>0.60</$ probability $>$

$<$ repCount $>10</$ repCount $>$

$<$ hostDemand $>(2.30, \underline{\mathrm{ms}})</$ hostDemand $>$

$</$ step $>$

<step xsi:type="ResourceRelease" name="rr: componentB" predecessor="//@scenario.0/@pathConnection.7"

successor="//@scenario.0/@pathConnection.5" release="//@resource.1"/>

<step xsi:type="ResourceRelease" name="rr: componentB" predecessor="//@scenario.0/@pathConnection.8"

successor="//@scenario.0/@pathConnection.9" release="//@resource.1"/>

<step xsi:type="End" name="end" predecessor="//@scenario.0/@pathConnection.9"/>

<step name="fragment init" predecessor="//@scenario.0/@pathConnection.6"

successor="//@scenario.0/@pathConnection.4"/>

<step xsi:type="ResourceRelease" name="rr: componentA" predecessor="//@scenario.0/@pathConnection.5"

successor="//@scenario.0/@pathConnection.10" release="//@resource.0"/>

<step xsi:type="End" name="end" predecessor="//@scenario.0/@pathConnection.10"/>

<pathConnection xsi:type="StepSequence" source="//@scenario.0/@step.0" target="//@scenario.0/@step.1"/> <pathConnection xsi:type="StepSequence" source="//@scenario.0/@step.5" target="//@scenario.0/@step.3"/> <pathConnection xsi:type="StepSequence" source="//@scenario.0/@step.3" target="//@scenario.0/@step.4"/> <pathConnection xsi:type="StepSequence" source="//@scenario.0/@step.6" target="//@scenario.0/@step.7"/> 
<pathConnection xsi:type="Branch" name="fragment" source="//@scenario.0/@step.11"

target="//@scenario.0/@step.6//@scenario.0/@step.2"/>

<pathConnection xsi:type="Merge" name="fragment" source="//@scenario.0/@step.8 //@scenario.0/@step.2"

target="//@scenario.0/@step.12"/>

<pathConnection xsi:type="Fork" name="op1" source="//@scenario.0/@step.1" target="//@scenario.0/@step.5

//@scenario.0/@step.11"/>

<pathConnection xsi:type="StepSequence" source="//@scenario.0/@step.7" target="//@scenario.0/@step.8"/>

<pathConnection xsi:type="StepSequence" source="//@scenario.0/@step.4" target="//@scenario.0/@step.9"/>

<pathConnection xsi:type="StepSequence" source="//@scenario.0/@step.9" target="//@scenario.0/@step.10"/>

<pathConnection xsi:type="StepSequence" source="//@scenario.0/@step.12" target="//@scenario.0/@step.13"/>

$<$ scenario $>$

$<$ workload $>$

$<$ arrivalPattern >closed</arrivalPattern >

$</$ workload $>$

$<$ resource xsi:type $=$ "Component" name $=$ "componentA" isActive $="$ false" $/>$

$<$ resource xsi:type $=$ "Component" name $="$ componentB" isActive $="$ true" $/>$

$<$ Model $>$

\section{$\underline{\text { A.2 Test Case \#1 }}$}

$<?$ xml version="1.0" encoding="ISO-8859-1"?>

<Model xmi:version="2.0" xmlns:xmi="http://www.omg.org/XMI" xmlns:xsi="http://www.w3.org/2001/XMLSchema-

instance" xmlns="platform:/resource/MM2CSM/Metamodels/CSM.ecore" name="MarteModel1" >

$<$ scenario name $=$ "MarteModel1" >

<step xsi:type="Start" name="start" successor="//@scenario.0/@pathConnection.0"/>

<step xsi:type="ResourceAcquire" name="ra: actor1" predecessor="//@scenario.0/@pathConnection.0"

successor="//@scenario.0/@pathConnection.9"/>

<step name="op3" predecessor="//@scenario.0/@pathConnection.16"

successor="//@scenario.0/@pathConnection.17"/>

<step xsi:type="ResourceAcquire" name="ra: component1" predecessor="//@scenario.0/@pathConnection.1" successor="//@scenario.0/@pathConnection.5"/>

<step xsi:type="ResourceAcquire" name="ra: component2" predecessor="//@scenario.0/@pathConnection.2" successor="//@scenario.0/@pathConnection.6"/>

<step xsi:type="ResourceAcquire" name="ra: component1" predecessor="//@scenario.0/@pathConnection.3"

successor="//@scenario.0/@pathConnection.7"/>

<step xsi:type="ResourceAcquire" name="ra: actor1" predecessor="//@scenario.0/@pathConnection.4"

successor="//@scenario.0/@pathConnection.8"/>

<step name="op1" predecessor="//@scenario.0/@pathConnection.5"

successor="//@scenario.0/@pathConnection.10"/>

<step name="op2" predecessor="//@scenario.0/@pathConnection.6"

successor="//@scenario.0/@pathConnection.11"/>

$<$ step name="op2return" predecessor="//@scenario.0/@pathConnection.7"

successor="//@scenario.0/@pathConnection.12"/>

<step name="op1return" predecessor="//@scenario.0/@pathConnection.8"

successor="//@scenario.0/@pathConnection.16"/>

<step xsi:type="ResourceRelease" name="rr: actor1" predecessor="//@scenario.0/@pathConnection.9" successor="//@scenario.0/@pathConnection.1"/>

<step xsi:type="ResourceRelease" name="rr: component1" predecessor="//@scenario.0/@pathConnection.10" successor="//@scenario.0/@pathConnection.2"/>

<step xsi:type="ResourceRelease" name="rr: component2" predecessor="//@scenario.0/@pathConnection.11" successor="//@scenario.0/@pathConnection.3"/> 
<step xsi:type="ResourceRelease" name="rr. component1" predecessor="//@scenario.0/@pathConnection.12" successor="//@scenario.0/@pathConnection.4"/>

<step xsi:type="ResourceRelease" name="rr: component1" predecessor="//@scenario.0/@pathConnection.10" successor="//@scenario.0/@pathConnection.13"/>

<step xsi:type="ResourceRelease" name="rr. component2" predecessor="//@scenario.0/@pathConnection.11"

successor="//@scenario.0/@pathConnection.14"/>

<step xsi:type="ResourceRelease" name="rr: component1" predecessor="//@scenario.0/@pathConnection.12"

successor="//@scenario.0/@pathConnection.15"/>

<step xsi:type="End" name="end" predecessor="//@scenario.0/@pathConnection.13"/>

<step xsi:type="End" name="end" predecessor="//@scenario.0/@pathConnection.14"/>

<step xsi:type="End" name="end" predecessor="//@scenario.0/@pathConnection.15"/>

<step name="join init" predecessor="//@scenario.0/@pathConnection.9"

successor="//@scenario.0/@pathConnection.16"/>

<step xsi:type="ResourceRelease" name="rr. actor1" predecessor="//@scenario.0/@pathConnection.17"

successor="//@scenario.0/@pathConnection.18"/>

<step xsi:type="End" name="end" predecessor="//@scenario.0/@pathConnection.18"/>

<pathConnection xsi:type="StepSequence" source="//@scenario.0/@step.0" target="//@scenario.0/@step.1"/>

<pathConnection xsi:type="StepSequence" source="//@scenario.0/@step.11" target="//@scenario.0/@step.3"/>

<pathConnection xsi:type="StepSequence" source="//@scenario.0/@step.12" target="//@scenario.0/@step.4"/>

<pathConnection xsi:type="StepSequence" source="//@scenario.0/@step.13" target="//@scenario.0/@step.5"/>

<pathConnection xsi:type="StepSequence" source="//@scenario.0/@step.14" target="//@scenario.0/@step.6"/>

<pathConnection xsi:type="StepSequence" source="//@scenario.0/@step.3" target="//@scenario.0/@step.7"/>

<pathConnection xsi:type="StepSequence" source="//@scenario.0/@step.4" target="//@scenario.0/@step.8"/>

<pathConnection xsi:type="StepSequence" source="//@scenario.0/@step.5" target="//@scenario.0/@step.9"/>

<pathConnection xsi:type="StepSequence" source="//@scenario.0/@step.6" target="//@scenario.0/@step.10"/>

<pathConnection xsi:type="Fork" name="op1" source="//@scenario.0/@step.1" target="//@scenario.0/@step.11

//@scenario.0/@step.21"/>

<pathConnection xsi:type="Fork" name="op2" source="//@scenario.0/@step.7" target="//@scenario.0/@step.12

//@scenario.0/@step.15"/>

$<$ pathConnection xsi:type="Fork" name="op2return" source="//@scenario.0/@step.8"

target="//@scenario.0/@step.13//@scenario.0/@step.16"/>

<pathConnection xsi:type="Fork" name="op1return" source="//@scenario.0/@step.9"

target="//@scenario.0/@step.14//@scenario.0/@step.17"/>

<pathConnection xsi:type="StepSequence" source="//@scenario.0/@step.15" target="//@scenario.0/@step.18"/> <pathConnection xsittype="StepSequence" source="//@scenario.0/@step.16" target="//@scenario.0/@step.19"/> <pathConnection xsi:type="StepSequence" source="//@scenario.0/@step.17" target="//@scenario.0/@step.20"/> <pathConnection xsi:type="Join" name="op1return" source="//@scenario.0/@step.21 //@scenario.0/@step.10"

target="//@scenario.0/@step.2"/>

<pathConnection xsi:type="StepSequence" source="//@scenario.0/@step.2" target="//@scenario.0/@step.22"/> <pathConnection xsi:type="StepSequence" source="//@scenario.0/@step.22" target="//@scenario.0/@step.23"/> $</$ scenario $>$

$<$ /Model $>$

\section{$\underline{\text { A.3 Test Case \#2 }}$}

$<? \underline{x m l}$ version="1.0" encoding="ISO-8859-1"? >

<Model xmi:version="2.0" xmlns:xmi="http://www.omg.org/XMI" xmlns:xsi="http://www.w3.org/2001/XMLSchema-

instance" xmlns="platform:/resource/MM2CSM/Metamodels/CSM.ecore" name="MarteModel2" >

$<$ scenario name $=$ "MarteModel2" >

<step xsi:type="Start" name="start" successor="//@scenario.0/@pathConnection.0"/> 
<step xsi:type="ResourceAcquire" name="ra: component1" predecessor="//@scenario.0/@pathConnection.0" successor="//@scenario.0/@pathConnection.11"/>

$<$ step name="op1" predecessor="//@scenario.0/@pathConnection.11"

successor="//@scenario.0/@pathConnection.9"/>

<step xsi:type="ResourceAcquire" name="ra: component2" predecessor="//@scenario.0/@pathConnection.1" successor="//@scenario.0/@pathConnection.5"/>

<step xsi:type="ResourceAcquire" name="ra: component2" predecessor="//@scenario.0/@pathConnection.2" successor="//@scenario.0/@pathConnection.6"/>

<step xsi:type="ResourceAcquire" name="ra: component3" predecessor="//@scenario.0/@pathConnection.3" successor="//@scenario.0/@pathConnection.7"/>

<step xsi:type="ResourceAcquire" name="ra: component3" predecessor="//@scenario.0/@pathConnection.4" successor="//@scenario.0/@pathConnection.8"/>

<step name="op2" predecessor="//@scenario.0/@pathConnection.5"

successor="//@scenario.0/@pathConnection.12"/>

<step name="op3" predecessor="//@scenario.0/@pathConnection.6"

successor="//@scenario.0/@pathConnection.13"/>

<step name="op4" predecessor="//@scenario.0/@pathConnection.7"

successor="//@scenario.0/@pathConnection.14"/>

<step name="op5" predecessor="//@scenario.0/@pathConnection.8"

successor="//@scenario.0/@pathConnection.15"/>

<step xsi:type="ResourceRelease" name="rrr: component1" predecessor="//@scenario.0/@pathConnection.9" successor="//@scenario.0/@pathConnection.1"/>

<step xsi:type="ResourceRelease" name="rr: component1" predecessor="//@scenario.0/@pathConnection.9" successor="//@scenario.0/@pathConnection.2"/>

<step xsi:type="ResourceRelease" name="rr: component1" predecessor="//@scenario.0/@pathConnection.9" successor="//@scenario.0/@pathConnection.3"/>

<step xsi:type="ResourceRelease" name="rr: component1" predecessor="//@scenario.0/@pathConnection.9" successor="//@scenario.0/@pathConnection.4"/>

<step xsi:type="ResourceRelease" name="rr: component1" predecessor="//@scenario.0/@pathConnection.9"

successor="//@scenario.0/@pathConnection.10"/>

<step xsi:type="End" name="end" predecessor="//@scenario.0/@pathConnection.10"/>

<step xsi:type="ResourceRelease" name="rr: component2" predecessor="//@scenario.0/@pathConnection.12"

successor="//@scenario.0/@pathConnection.16"/>

<step xsi:type="ResourceRelease" name="rr: component2" predecessor="//@scenario.0/@pathConnection.13"

successor="//@scenario.0/@pathConnection.17"/>

<step xsi:type="ResourceRelease" name="r: component3" predecessor="//@scenario.0/@pathConnection.14"

successor="//@scenario.0/@pathConnection.18"/>

<step xsi:type="ResourceRelease" name="rr: component3" predecessor="//@scenario.0/@pathConnection.15"

successor="//@scenario.0/@pathConnection.19"/>

<step xsi:type="End" name="end" predecessor="//@scenario.0/@pathConnection.16"/>

<step xsi:type="End" name="end" predecessor="//@scenario.0/@pathConnection.17"/>

<step xsi:type="End" name="end" predecessor="//@scenario.0/@pathConnection.18"/>

<step xsi:type="End" name="end" predecessor="//@scenario.0/@pathConnection.19"/>

<pathConnection xsi:type="StepSequence" source="//@scenario.0/@step.0" target="//@scenario.0/@step.1"/>

<pathConnection xsi:type="StepSequence" source="//@scenario.0/@step.11" target="//@scenario.0/@step.3"/> <pathConnection xsi:type="StepSequence" source="//@scenario.0/@step.12" target="//@scenario.0/@step.4"/> <pathConnection xsi:type="StepSequence" source="//@scenario.0/@step.13" target="//@scenario.0/@step.5"/> <pathConnection xsi:type="StepSequence" source="//@scenario.0/@step.14" target="//@scenario.0/@step.6"/> <pathConnection xsi:type="StepSequence" source="//@scenario.0/@step.3" target="//@scenario.0/@step.7"/> <pathConnection xsi:type="StepSequence" source="//@scenario.0/@step.4" target="//@scenario.0/@step.8"/> <pathConnection xsi:type="StepSequence" source="//@scenario.0/@step.5" target="//@scenario.0/@step.9"/> <pathConnection xsi:type="StepSequence" source="//@scenario.0/@step.6" target="//@scenario.0/@step.10"/> <pathConnection xsi:type="Fork" name="op2" source="//@scenario.0/@step.2" target="//@scenario.0/@step.11 //@scenario.0/@step.12 //@scenario.0/@step.13 //@scenario.0/@step.14"/> 
<pathConnection xsi:type="StepSequence" source="//@scenario.0/@step.15" target="//@scenario.0/@step.16"/> <pathConnection xsi:type="StepSequence" source="//@scenario.0/@step.1" target="//@scenario.0/@step.2"/> <pathConnection xsi:type="StepSequence" source="//@scenario.0/@step.7" target="//@scenario.0/@step.17"/> <pathConnection xsi:type="StepSequence" source="//@scenario.0/@step.8" target="//@scenario.0/@step.18"/> <pathConnection xsi:type="StepSequence" source="//@scenario.0/@step.9" target="//@scenario.0/@step.19"/> <pathConnection xsitype="StepSequence" source="//@scenario.0/@step.10" target="//@scenario.0/@step.20"/> <pathConnection xsittype="StepSequence" source="//@scenario.0/@step.17" target="//@scenario.0/@step.21"/> <pathConnection xsi:type="StepSequence" source="//@scenario.0/@step.18" target="//@scenario.0/@step.22"/> <pathConnection xsi:type="StepSequence" source="//@scenario.0/@step.19" target="//@scenario.0/@step.23"/> <pathConnection xsi:type="StepSequence" source="//@scenario.0/@step.20" target="//@scenario.0/@step.24"/> $</$ scenario $>$

$<$ /Model $>$

\section{$\underline{\text { A.4 Test Case \#3 }}$}

$<?$ xml version="1.0" encoding="ISO-8859-1"?>

<Model xmi:version="2.0" xmlns:xmi="http://www.omg.org/XMI" xmlns:xsi="http://www.w3.org/2001/XMLSchema-

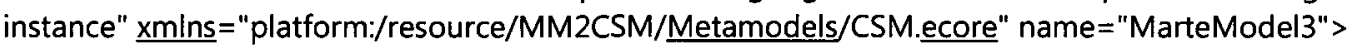

$\langle$ scenario name $=$ "MarteModel3" >

<step xsi:type="Start" name="start" successor="//@scenario.0/@pathConnection.0"/>

<step xsi:type="ResourceAcquire" name="ra: component1" predecessor="//@scenario.0/@pathConnection.0"

successor="//@scenario.0/@pathConnection.11"/>

<step xsi:type="ResourceAcquire" name="ra: component2" predecessor="//@scenario.0/@pathConnection.1" successor="//@scenario.0/@pathConnection.5"/>

<step xsi:type="ResourceAcquire" name="ra: component2" predecessor="//@scenario.0/@pathConnection.2" successor="//@scenario.0/@pathConnection.6"/>

<step xsi:type="ResourceAcquire" name="ra: component2" predecessor="//@scenario.0/@pathConnection.3" successor="//@scenario.0/@pathConnection.7"/>

<step xsi:type="ResourceAcquire" name="ra: component2" predecessor="//@scenario.0/@pathConnection.4" successor="//@scenario.0/@pathConnection.8"/>

<step name="op1" predecessor="//@scenario.0/@pathConnection.5"

successor="//@scenario.0/@pathConnection.16"/>

<step name="op2" predecessor="//@scenario.0/@pathConnection.6"

successor="//@scenario.0/@pathConnection.17"/>

<step name="op3" predecessor="//@scenario.0/@pathConnection.7"

successor="//@scenario.0/@pathConnection.18"/>

<step name="op4" predecessor="//@scenario.0/@pathConnection.8"

successor="//@scenario.0/@pathConnection.19"/>

<step xsi:type="ResourceRelease" name="rr. component1" predecessor="//@scenario.0/@pathConnection.11" successor="//@scenario.0/@pathConnection.1"/>

<step xsi:type="ResourceRelease" name="r: component1" predecessor="//@scenario.0/@pathConnection.12" successor="//@scenario.0/@pathConnection.2"/>

<step xsi:type="ResourceRelease" name="rr: component1" predecessor="//@scenario.0/@pathConnection.13" successor="//@scenario.0/@pathConnection.3"/>

<step xsi:type="ResourceRelease" name="rr. component1" predecessor="//@scenario.0/@pathConnection.14"

successor="//@scenario.0/@pathConnection.4"/>

<step xsi:type="ResourceRelease" name="rr: component1" predecessor="//@scenario.0/@pathConnection.14"

successor="//@scenario.0/@pathConnection.15"/>

<step xsi:type="End" name="end" predecessor="//@scenario.0/@pathConnection.15"/>

<step xsi:type="ResourceRelease" name="rr: component2" predecessor="//@scenario.0/@pathConnection.16" successor="//@scenario.0/@pathConnection.20"/> 
<step xsi:type="ResourceRelease" name="r: component2" predecessor="//@scenario.0/@pathConnection.17" successor="//@scenario.0/@pathConnection.21"/>

<step xsi:type="ResourceRelease" name="rr: component2" predecessor="//@scenario.0/@pathConnection.18" successor="//@scenario.0/@pathConnection.22"/>

<step xsi:type="ResourceRelease" name="rr: component2" predecessor="//@scenario.0/@pathConnection.19"

successor="//@scenario.0/@pathConnection.23"/>

<step xsi:type="End" name="end" predecessor="//@scenario.0/@pathConnection.20"/>

<step xsi:type="End" name="end" predecessor="//@scenario.0/@pathConnection.21"/>

<step xsi:type="End" name="end" predecessor="//@scenario.0/@pathConnection.22"/>

<step xsi:type="End" name="end" predecessor="//@scenario.0/@pathConnection.23"/>

<step name="alt init" predecessor="//@scenario.0/@pathConnection.11"

successor="//@scenario.0/@pathConnection.9"/>

<step name="alt end" predecessor="//@scenario.0/@pathConnection.10"

successor="//@scenario.0/@pathConnection.14"/>

<step name="alt1 init" predecessor="//@scenario.0/@pathConnection.9"

successor="//@scenario.0/@pathConnection.12"/>

<step name="alt2 init" predecessor="//@scenario.0/@pathConnection.9"

successor="//@scenario.0/@pathConnection.13"/>

<step name="alt1 end" predecessor="//@scenario.0/@pathConnection.12"

successor="//@scenario.0/@pathConnection.10"/>

<step name="alt2 end" predecessor="//@scenario.0/@pathConnection.13"

successor="//@scenario.0/@pathConnection.10"/>

<pathConnection xsi:type="StepSequence" source="//@scenario.0/@step.0" target="//@scenario.0/@step.1"/> <pathConnection xsi:type="StepSequence" source="//@scenario.0/@step.10" target="//@scenario.0/@step.2"/> <pathConnection xsi:type="StepSequence" source="//@scenario.0/@step.11" target="//@scenario.0/@step.3"/> <pathConnection xsi:type="StepSequence" source="//@scenario.0/@step.12" target="//@scenario.0/@step.4"/> <pathConnection xsi:type="StepSequence" source="//@scenario.0/@step.13" target="//@scenario.0/@step.5"/> <pathConnection xsi:type="StepSequence" source="//@scenario.0/@step.2" target="//@scenario.0/@step.6"/> <pathConnection xsi:type="StepSequence" source="//@scenario.0/@step.3" target="//@scenario.0/@step.7"/> <pathConnection xsi:type="StepSequence" source="//@scenario.0/@step.4" target="//@scenario.0/@step.8"/> <pathConnection xsi:type="StepSequence" source="//@scenario.0/@step.5" target="//@scenario.0/@step.9"/> <pathConnection xsi:type="Branch" name="alt" source="//@scenario.0/@step.24"

target="//@scenario.0/@step.26//@scenario.0/@step.27"/>

<pathConnection xsi:type="Merge" name="alt" source="//@scenario.0/@step.28 //@scenario.0/@step.29"

target="//@scenario.0/@step.25"/>

<pathConnection xsi:type="Fork" name="op1" source="//@scenario.0/@step.1" target="//@scenario.0/@step.10

//@scenario.0/@step.24"/>

<pathConnection xsi:type="Fork" name="op2" source="//@scenario.0/@step.26" target="//@scenario.0/@step.11 //@scenario.0/@step.28"/>

<pathConnection xsi:type="Fork" name="op3" source="//@scenario.0/@step.27" target="//@scenario.0/@step.12 //@scenario.0/@step.29"/>

<pathConnection xsi:type="Fork" name="op4" source="//@scenario.0/@step.25" target="//@scenario.0/@step.13 //@scenario.0/@step.14"/>

<pathConnection xsi:type="StepSequence" source="//@scenario.0/@step.14" target="//@scenario.0/@step.15"/> <pathConnection xsi:type="StepSequence" source="//@scenario.0/@step.6" target="//@scenario.0/@step.16"/> <pathConnection xsi:type="StepSequence" source="//@scenario.0/@step.7"target="//@scenario.0/@step.17"/> <pathConnection xsi:type="StepSequence" source="//@scenario.0/@step.8" target="//@scenario.0/@step.18"/> <pathConnection xsi:type="StepSequence" source="//@scenario.0/@step.9" target="//@scenario.0/@step.19"/> <pathConnection xsi:type="StepSequence" source="//@scenario.0/@step.16" target="//@scenario.0/@step.20"/> <pathConnection xsi:type="StepSequence" source="//@scenario.0/@step.17" target="//@scenario.0/@step.21"/> <pathConnection xsi:type="StepSequence" source="//@scenario.0/@step.18" target="//@scenario.0/@step.22"/> <pathConnection xsi:type="StepSequence" source="//@scenario.0/@step.19" target="//@scenario.0/@step.23"/> $<$ /scenarios

</Model> 


\section{A.5 Test Case \#4}

$<? \mathrm{xml}$ version="1.0" encoding="ISO-8859-1"?> <Model xmi:version="2.0" xmlns:xmi="http://www.omg.org/XMI" xmlns:xsi="http://www.w3.org/2001/XMLSchemainstance" $\underline{x m l n s}=$ "platform:/resource/MM2CSM/Metamodels/CSM.ecore" name="MarteModel4" > $<$ scenario name $=$ "MarteModel4" > <step name="op1" predecessor="//@scenario.0/@pathConnection.3" successor="//@scenario.0/@pathConnection.4"/> <step name="op3" predecessor="//@scenario.0/@pathConnection.1" successor="//@scenario.0/@pathConnection.2"/> <step name="op4" predecessor="//@scenario.0/@pathConnection.1" successor="//@scenario.0/@pathConnection.2"/> <step xsi:type="ResourceAcquire" name="ra: component2" predecessor="//@scenario.0/@pathConnection.3" successor="//@scenario.0/@pathConnection.0"/> <step name="op2" predecessor="//@scenario.0/@pathConnection.0" successor="//@scenario.0/@pathConnection.5"/> <step xsi:type="ResourceRelease" name="rr: component2" predecessor="//@scenario.0/@pathConnection.5" successor="//@scenario.0/@pathConnection.4"/> <step name="alt init" predecessor="//@scenario.0/@pathConnection.4" successor="//@scenario.0/@pathConnection.1"/> <step xsi:type="Start" name="start" successor="//@scenario.0/@pathConnection.6"/> <step xsi:type="ResourceAcquire" name="ra: component1" predecessor="//@scenario.0/@pathConnection.6" successor="//@scenario.0/@pathConnection.3"/> <step xsi:type="ResourceRelease" name="rr: component1" predecessor="//@scenario.0/@pathConnection.2" successor="//@scenario.0/@pathConnection.7"/> <step xsi:type="End" name="end" predecessor="//@scenario.0/@pathConnection.7"/> <pathConnection xsi:type="StepSequence" source="//@scenario.0/@step.3" target="//@scenario.0/@step.4"/> <pathConnection xsi:type="Branch" name="alt" source="//@scenario.0/@step.6" target="//@scenario.0/@step.1 //@scenario.0/@step.2"/> <pathConnection xsi:type="Merge" name="alt" source="//@scenario.0/@step.1 //@scenario.0/@step.2" target="//@scenario.0/@step.9"/> <pathConnection xsi:type="Fork" name="par" source="//@scenario.0/@step.8" target="//@scenario.0/@step.0 //@scenario.0/@step.3"/> <pathConnection xsi:type="Join" name="par" source="//@scenario.0/@step.0 //@scenario.0/@step.5" target="//@scenario.0/@step.6"/> <pathConnection xsi:type="StepSequence" source="//@scenario.0/@step.4" target="//@scenario.0/@step.5"/> <pathConnection xsi:type="StepSequence" source="//@scenario.0/@step.7" target="//@scenario.0/@step.8"/> <pathConnection xsi:type="StepSequence" source="//@scenario.0/@step.9" target="//@scenario.0/@step.10"/> $<$ /scenario $>$

$<$ Model>

\section{$\underline{\text { A.6 Test Case \#5 }}$}

$<? \underline{x m l}$ version="1.0" encoding="ISO-8859-1"?> $<$ Model xmi:version="2.0" xmlns:xmi="http://www.omg.org/XMI" xmlns:xsi="http://www.w3.org/2001/XMLSchemainstance" xmlns="platform:/resource/MM2CSM/Metamodels/CSM.ecore" name="MarteModel5" > <scenario name="MarteModel5" >

<step name="op8" predecessor="//@scenario.0/@pathConnection.10" successor="//@scenario.0/@pathConnection.15"/> 
<step xsi:type="ResourceAcquire" name=" ra: component2" predecessor="//@scenario.0/@pathConnection.0" successor="//@scenario.0/@pathConnection.2"/>

<step xsi:type="ResourceAcquire" name="ra: component2" predecessor="//@scenario.0/@pathConnection.1" successor="//@scenario.0/@pathConnection.3"/>

<step name="op7" predecessor="//@scenario.0/@pathConnection.2"

successor="//@scenario.0/@pathConnection.25"/>

<step name="op6" predecessor="//@scenario.0/@pathConnection.3"

successor="//@scenario.0/@pathConnection.26"/>

<step xsi:type="ResourceRelease" name="rr. component1" predecessor="//@scenario.0/@pathConnection.20" successor="//@scenario.0/@pathConnection.0"/>

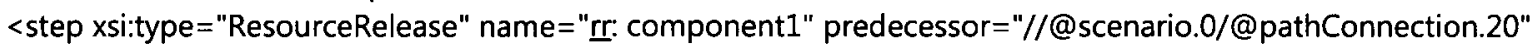
successor="//@scenario.0/@pathConnection.1"/>

<step xsi:type="ResourceAcquire" name="ra: component2" predecessor="//@scenario.0/@pathConnection.8" successor="//@scenario.0/@pathConnection.4"/>

<step xsi:type="ResourceAcquire" name="ra: component2" predecessor="//@scenario.0/@pathConnection.8" successor="//@scenario.0/@pathConnection.5"/>

<step xsi:type="ResourceAcquire" name="ra: component2" predecessor="//@scenario.0/@pathConnection.9" successor="//@scenario.0/@pathConnection.6"/>

<step xsi:type="ResourceAcquire" name="ra: component2" predecessor="//@scenario.0/@pathConnection.9" successor="//@scenario.0/@pathConnection.7"/>

<step name="op1" predecessor="//@scenario.0/@pathConnection.4"

successor="//@scenario.0/@pathConnection.21"/>

<step name="op2" predecessor="//@scenario.0/@pathConnection.5"

successor="//@scenario.0/@pathConnection.22"/>

<step name="op3" predecessor="//@scenario.0/@pathConnection.6"

successor="//@scenario.0/@pathConnection.23"/>

<step name="op4" predecessor="//@scenario.0/@pathConnection.7"

successor="//@scenario.0/@pathConnection.24"/>

<step xsi:type="ResourceRelease" name="rr: component2" predecessor="//@scenario.0/@pathConnection.21"

successor="//@scenario.0/@pathConnection.13"/>

<step xsi:type="ResourceRelease" name="rir: component2" predecessor="//@scenario.0/@pathConnection.22"

successor="//@scenario.0/@pathConnection.13"/>

<step xsi:type="ResourceRelease" name="rr: component2" predecessor="//@scenario.0/@pathConnection.23"

successor="//@scenario.0/@pathConnection.14"/>

<step xsi:type="ResourceRelease" name="rr: component2" predecessor="//@scenario.0/@pathConnection.24"

successor="//@scenario.0/@pathConnection.14"/>

<step xsi:type="ResourceRelease" name="rr: component2" predecessor="//@scenario.0/@pathConnection.25"

successor="//@scenario.0/@pathConnection.27"/>

<step xsi:type="ResourceRelease" name="rr: component2" predecessor="//@scenario.0/@pathConnection.26"

successor="//@scenario.0/@pathConnection.28"/>

<step xsi:type="End" name="end" predecessor="//@scenario.0/@pathConnection.27"/>

<step xsi:type="End" name="end" predecessor="//@scenario.0/@pathConnection.28"/>

$<$ step name="alt1 init" predecessor="//@scenario.0/@pathConnection.18"

successor="//@scenario.0/@pathConnection.8"/>

<step name="alt2 init" predecessor="//@scenario.0/@pathConnection.18"

successor="//@scenario.0/@pathConnection.9"/>

<step name="alt3 init" predecessor="//@scenario.0/@pathConnection.19"

successor="//@scenario.0/@pathConnection.10"/>

<step name="alt4 init" predecessor="//@scenario.0/@pathConnection.10"

successor="//@scenario.0/@pathConnection.11"/>

<step name="alt5 init" predecessor="//@scenario.0/@pathConnection.11"

successor="//@scenario.0/@pathConnection.12"/>

<step name="alt1 end" predecessor="//@scenario.0/@pathConnection.13"

successor="//@scenario.0/@pathConnection.19"/> 
<step name="alt2 end" predecessor="//@scenario.0/@pathConnection.14"

successor="//@scenario.0/@pathConnection.19"/>

<step name="alt4 end" predecessor="//@scenario.0/@pathConnection.16"

successor="//@scenario.0/@pathConnection.15"/>

<step name="alt5 end" predecessor="//@scenario.0/@pathConnection.17"

successor="//@scenario.0/@pathConnection.16"/>

<step xsi:type="Start" name="start" successor="//@scenario.0/@pathConnection.29"/>

<step xsi:type="ResourceAcquire" name="ra: component1" predecessor="//@scenario.0/@pathConnection.29"

successor="//@scenario.0/@pathConnection.18"/>

<step xsi:type="ResourceRelease" name="rr: component1" predecessor="//@scenario.0/@pathConnection.15"

successor="//@scenario.0/@pathConnection.30"/>

<step xsi:type="End" name="end" predecessor="//@scenario.0/@pathConnection.30"/>

<step name="alt5a init" predecessor="//@scenario.0/@pathConnection.12"

successor="//@scenario.0/@pathConnection.20"/>

<step name="alt5a end" predecessor="//@scenario.0/@pathConnection.20"

successor="//@scenario.0/@pathConnection.17"/>

<pathConnection xsi:type="StepSequence" source="//@scenario.0/@step.5" target="//@scenario.0/@step.1"/>

<pathConnection xsi:type="StepSequence" source="//@scenario.0/@step.6" target="//@scenario.0/@step.2"/>

<pathConnection xsi:type="StepSequence" source="//@scenario.0/@step.1" target="//@scenario.0/@step.3"/>

<pathConnection xsi:type="StepSequence" source="//@scenario.0/@step.2" target="//@scenario.0/@step.4"/>

<pathConnection xsi:type="StepSequence" source="//@scenario.0/@step.7"target="//@scenario.0/@step.11"/>

<pathConnection xsittype="StepSequence" source="//@scenario.0/@step.8" target="//@scenario.0/@step.12"/>

<pathConnection xsittype="StepSequence" source="//@scenario.0/@step.9" target="//@scenario.0/@step.13"/>

<pathConnection xsi:type="StepSequence" source="//@scenario.0/@step.10" target="//@scenario.0/@step.14"/>

$<$ pathConnection xsi:type="Branch" name="alt1" source="//@scenario.0/@step.23"

target="//@scenario.0/@step.7//@scenario.0/@step.8"/>

<pathConnection xsi:type="Branch" name="alt2" source="//@scenario.0/@step.24"

target="//@scenario.0/@step.9//@scenario.0/@step.10"/>

<pathConnection xsi:type="Branch" name="alt3" source="//@scenario.0/@step.25"

target="//@scenario.0/@step.26//@scenario.0/@step.0"/>

<pathConnection xsi:type="Branch" name="alt4" source="//@scenario.0/@step.26"

target="//@scenario.0/@step.27"/>

<pathConnection xsi:type="Branch" name="alt5" source="//@scenario.0/@step.27"

target="//@scenario.0/@step.36"/>

<pathConnection xsi:type="Merge" name="alt1" source="//@scenario.0/@step.15 //@scenario.0/@step.16"

target="//@scenario.0/@step.28"/>

<pathConnection xsi:type="Merge" name="alt2" source="//@scenario.0/@step.17 //@scenario.0/@step.18"

target="//@scenario.0/@step.29"/>

<pathConnection xsi:type="Merge" name="alt3" source="//@scenario.0/@step.30 //@scenario.0/@step.0"

target="//@scenario.0/@step.34"/>

<pathConnection xsi:type="Merge" name="alt4" source="//@scenario.0/@step.31"

target="//@scenario.0/@step.30"/>

<pathConnection xsi:type="Merge" name="alt5" source="//@scenario.0/@step.37"

target="//@scenario.0/@step.31"/>

<pathConnection xsi:type="Fork" name="par1" source="//@scenario.0/@step.33" target="//@scenario.0/@step.23

//@scenario.0/@step.24"/>

<pathConnection xsi:type="Join" name="par1" source="//@scenario.0/@step.28 //@scenario.0/@step.29"

target="//@scenario.0/@step.25"/>

<pathConnection xsi:type="Fork" name="op6" source="//@scenario.0/@step.36" target="//@scenario.0/@step.6

//@scenario.0/@step.37//@scenario.0/@step.5"/>

<pathConnection xsi:type="StepSequence" source="//@scenario.0/@step.11" target="//@scenario.0/@step.15"/> <pathConnection xsi:type="StepSequence" source="//@scenario.0/@step.12" target="//@scenario.0/@step.16"/> <pathConnection xsi:type="StepSequence" source="//@scenario.0/@step.13" target="//@scenario.0/@step.17"/> <pathConnection xsi:type="StepSequence" source="//@scenario.0/@step.14" target="//@scenario.0/@step.18"/> 
<pathConnection xsi:type="StepSequence" source="//@scenario.0/@step.3" target="//@scenario.0/@step.19"/> <pathConnection xsi:type="StepSequence" source="//@scenario.0/@step.4" target="//@scenario.0/@step.20"/> <pathConnection xsi:type="StepSequence" source="//@scenario.0/@step.19" target="//@scenario.0/@step.21"/> <pathConnection xsi:type="StepSequence" source="//@scenario.0/@step.20" target="//@scenario.0/@step.22"/> <pathConnection xsi:type="StepSequence" source="//@scenario.0/@step.32" target="//@scenario.0/@step.33"/> <pathConnection xsi:type="StepSequence" source="//@scenario.0/@step.34" target="//@scenario.0/@step.35"/> $</$ scenario $>$

$<$ Model $>$

\section{$\underline{\text { A.7 Test Case \#6 }}$}

$<? \underline{x m l}$ version="1.0" encoding ="ISO-8859-1"?>

<Model xmi:version="2.0" xmlns:xmi="http://www.omg.org/XMI" xmlns:xsi="http://www.w3.org/2001/XMLSchemainstance" xmlns="platform:/resource/MM2CSM/Metamodels/CSM.ecore" name="MarteModel6" >

<scenario name="MarteModel6">

<step name="loop" predecessor="//@scenario.0/@pathConnection.0"

successor="//@scenario.0/@pathConnection.1" refinement="//@refinement.0"/>

<step xsi:type="Start" name="start" successor="//@scenario.0/@pathConnection.2"/>

<step xsi:type="ResourceAcquire" name="aㅡ: component1" predecessor="//@scenario.0/@pathConnection.2"

successor="//@scenario.0/@pathConnection.0"/>

<step xsi:type="ResourceRelease" name="rr: component1" predecessor="//@scenario.0/@pathConnection.1"

successor="//@scenario.0/@pathConnection.3"/>

<step xsi:type="End" name="end" predecessor="//@scenario.0/@pathConnection.3"/>

$<$ pathConnection xsi:type="Branch" name="alt" source="//@scenario.0/@step.2"

target="//@scenario.0/@step.0"/>

<pathConnection xsi:type="Merge" name="alt" source="//@scenario.0/@step.0"

target="//@scenario.0/@step.3"/>

<pathConnection xsi:type="StepSequence" source="//@scenario.0/@step.1" target="//@scenario.0/@step.2"/>

<pathConnection xsi:type="StepSequence" source="//@scenario.0/@step.3" target="//@scenario.0/@step.4"/>

$<$ scenario $>$

<scenario name="loop" parent="//@scenario.0/@step.0">

<step xsi:type="Start" name="loop start" successor="//@scenario.1/@pathConnection.8"/>

<step xsi:type="ResourceAcquire" name="ra: component2" predecessor="//@scenario.1/@pathConnection.0"

successor="//@scenario.1/@pathConnection.2"/>

<step xsi:type="ResourceAcquire" name="ra: component2" predecessor="//@scenario.1/@pathConnection.1"

successor="//@scenario.1/@pathConnection.3"/>

<step name="op2" predecessor="//@scenario.1/@pathConnection.2"

successor="//@scenario.1/@pathConnection.6"/>

<step name="op3" predecessor="//@scenario.1/@pathConnection.3"

successor="//@scenario.1/@pathConnection.6"/>

<step xsi:type="ResourceRelease" name="rr. component1" predecessor="//@scenario.1/@pathConnection.5"

successor="//@scenario.1/@pathConnection.0"/>

<step xsi:type="ResourceRelease" name="rr: component1" predecessor="//@scenario.1/@pathConnection.5"

successor="//@scenario.1/@pathConnection.1"/>

<step xsi:type="ResourceAcquire" name="ra: component2" predecessor="//@scenario.1/@pathConnection.8"

successor="//@scenario.1/@pathConnection.4"/>

<step name="op1" predecessor="//@scenario.1/@pathConnection.4"

successor="//@scenario.1/@pathConnection.9"/>

<step xsi:type="ResourceRelease" name="rr: component2" predecessor="//@scenario.1/@pathConnection.9" successor="//@scenario.1/@pathConnection.5"/> 
<step xsi:type="ResourceRelease" name="rrr: component2" predecessor="//@scenario.1/@pathConnection.6" successor="//@scenario.1/@pathConnection.7"/>

<step xsi:type="End" name="end" predecessor="//@scenario.1/@pathConnection.7"/>

<step xsi:type="End" name="loop end" predecessor="//@scenario.1/@pathConnection.5"/>

<pathConnection xsi:type="StepSequence" source="//@scenario.1/@step.5" target="//@scenario.1/@step.1"/> <pathConnection xsi:type="StepSequence" source="//@scenario.1/@step.6" target="//@scenario.1/@step.2"/> <pathConnection xsi:type="StepSequence" source="//@scenario.1/@step.1" target="//@scenario.1/@step.3"/> <pathConnection xsi:type="StepSequence" source="//@scenario.1/@step.2" target="//@scenario.1/@step.4"/> <pathConnection xsi:type="StepSequence" source="//@scenario.1/@step.7" target="//@scenario.1/@step.8"/> <pathConnection xsi:type="Fork" name="op2" source="//@scenario.1/@step.9" target="//@scenario.1/@step.5 //@scenario.1/@step.12 //@scenario.1/@step.6"/>

<pathConnection xsi:type="Join" name="op2" source="//@scenario.1/@step.9 //@scenario.1/@step.3

//@scenario.1/@step.4" target="//@scenario.1/@step.10"/>

<pathConnection xsi:type="StepSequence" source="//@scenario.1/@step.10"target="//@scenario.1/@step.11"/> <pathConnection xsi:type="StepSequence" source="//@scenario.1/@step.0" target="//@scenario.1/@step.7"/> $<$ pathConnection xsi:type="StepSequence" source="//@scenario.1/@step.8" target="//@scenario.1/@step.9"/> $</$ scenario $>$ $<$ refinement parent="//@scenario.0/@step.0"sub="//@scenario.1"/> $<$ /Model $>$

\section{A.8 PUMA Example}

$<?$ xml version="1.0" encoding="ISO-8859-1"?>

<Model xmi:version="2.0" xmlns:xmi="http://www.omg.org/XMI" xmlns:xsi="http://www.w3.org/2001/XMLSchemainstance" $\mathrm{xmlns}=$ "platform:/resource/MM2CSM/Metamodels/CSM.ecore" name="PUMAExample" >

<scenario name $=$ "PUMAExample" >

<step xsi:type="Start" name="start" successor="//@scenario.0/@pathConnection.0"/>

<step xsi:type="ResourceAcquire" name="ra: Server" predecessor="//@scenario.0/@pathConnection.0"

successor="//@scenario.0/@pathConnection.1"/>

<step name="request" predecessor="//@scenario.0/@pathConnection.1"

successor="//@scenario.0/@pathConnection.6"/>

<step name="output" predecessor="//@scenario.0/@pathConnection.2"

successor="//@scenario.0/@pathConnection.3"/>

<step name="reply" predecessor="//@scenario.0/@pathConnection.3"

successor="//@scenario.0/@pathConnection.4"/>

<step name="loop" predecessor="//@scenario.0/@pathConnection.6"

successor="//@scenario.0/@pathConnection.2" refinement="//@refinement.0"/>

<step xsi:type="ResourceRelease" name="rr: Server" predecessor="//@scenario.0/@pathConnection.4"

successor="//@scenario.0/@pathConnection.5"/>

<step xsi:type="End" name="end" predecessor="//@scenario.0/@pathConnection.5"/>

<pathConnection xsi:type="StepSequence" source="//@scenario.0/@step.0" target="//@scenario.0/@step.1"/> <pathConnection xsi:type="StepSequence" source="//@scenario.0/@step.1" target="//@scenario.0/@step.2"/> <pathConnection xsi:type="StepSequence" source="//@scenario.0/@step.5" target="//@scenario.0/@step.3"/> <pathConnection xsi:type="StepSequence" source="//@scenario.0/@step.3" target="//@scenario.0/@step.4"/> <pathConnection xsi:type="StepSequence" source="//@scenario.0/@step.4" target="//@scenario.0/@step.6"/> <pathConnection xsi:type="StepSequence" source="//@scenario.0/@step.6" target="//@scenario.0/@step.7"/> <pathConnection xsi:type="StepSequence" source="//@scenario.0/@step.2" target="//@scenario.0/@step.5"/> $</$ scenario $>$

<scenario name="loop" parent="//@scenario.0/@step.5">

<step xsi:type="Start" name="loop start" successor="//@scenario.1/@pathConnection.11"/> 
<step name="init" predecessor="//@scenario.1/@pathConnection.11"

successor="//@scenario.1/@pathConnection.6"/>

<step xsi:type="ResourceAcquire" name="ra: DB1" predecessor="//@scenario.1/@pathConnection.0"

successor="//@scenario.1/@pathConnection.1"/>

<step name="log" predecessor="//@scenario.1/@pathConnection.1"

successor="//@scenario.1/@pathConnection.15"/>

<step xsi:type="ResourceRelease" name="rr: App" predecessor="//@scenario.1/@pathConnection.10"

successor="//@scenario.1/@pathConnection.0"/>

<step xsi:type="ResourceAcquire" name="ra: App" predecessor="//@scenario.1/@pathConnection.6"

successor="//@scenario.1/@pathConnection.2"/>

<step xsi:type="ResourceAcquire" name="ra: DB1" predecessor="//@scenario.1/@pathConnection.8"

successor="//@scenario.1/@pathConnection.3"/>

<step xsi:type="ResourceAcquire" name="ra: DB2" predecessor="//@scenario.1/@pathConnection.10"

successor="//@scenario.1/@pathConnection.4"/>

<step xsi:type="ResourceAcquire" name="ra: Cache" predecessor="//@scenario.1/@pathConnection.6"

successor="//@scenario.1/@pathConnection.5"/>

<step name="getData" predecessor="//@scenario.1/@pathConnection.2"

successor="//@scenario.1/@pathConnection.8"/>

<step name="read1" predecessor="//@scenario.1/@pathConnection.3"

successor="//@scenario.1/@pathConnection.13"/>

<step name="read2" predecessor="//@scenario.1/@pathConnection.4"

successor="//@scenario.1/@pathConnection.14"/>

<step name="readCache" predecessor="//@scenario.1/@pathConnection.5"

successor="//@scenario.1/@pathConnection.12"/>

<step xsi:type="ResourceRelease" name="rr: Cache" predecessor="//@scenario.1/@pathConnection.12"

successor="//@scenario.1/@pathConnection.7"/>

<step xsi:type="ResourceRelease" name="rr: DB1" predecessor="//@scenario.1/@pathConnection.13"

successor="//@scenario.1/@pathConnection.9"/>

<step xsi:type="ResourceRelease" name="rr: App" predecessor="//@scenario.1/@pathConnection.9"

successor="//@scenario.1/@pathConnection.7"/>

<step xsi:type="ResourceRelease" name="rr: DB2" predecessor="//@scenario.1/@pathConnection.14"

successor="//@scenario.1/@pathConnection.9"/>

<step xsi:type="ResourceRelease" name="rr: DB1" predecessor="//@scenario.1/@pathConnection.15"

successor="//@scenario.1/@pathConnection.16"/>

<step xsi:type="End" name="end" predecessor="//@scenario.1/@pathConnection.16"/>

<step name="par2 init" predecessor="//@scenario.1/@pathConnection.8"

successor="//@scenario.1/@pathConnection.10"/>

<step xsi:type="End" name="loop end" predecessor="//@scenario.1/@pathConnection.7"/>

<pathConnection xsi:type="StepSequence" source="//@scenario.1/@step.4" target="//@scenario.1/@step.2"/>

<pathConnection xsi:type="StepSequence" source="//@scenario.1/@step.2" target="//@scenario.1/@step.3"/>

<pathConnection xsi:type="StepSequence" source="//@scenario.1/@step.5" target="//@scenario.1/@step.9"/>

<pathConnection xsi:type="StepSequence" source="//@scenario.1/@step.6" target="//@scenario.1/@step.10"/>

<pathConnection xsi:type="StepSequence" source="//@scenario.1/@step.7" target="//@scenario.1/@step.11"/>

<pathConnection xsi:type="StepSequence" source="//@scenario.1/@step.8" target="//@scenario.1/@step.12"/>

<pathConnection xsi:type="Branch" name=" alt" source="//@scenario.1/@step.1" target="//@scenario.1/@step.8

//@scenario.1/@step.5"/>

<pathConnection xsi:type="Merge" name="alt" source="//@scenario.1/@step.13 //@scenario.1/@step.15"

target="//@scenario.1/@step.20"/>

<pathConnection xsi:type="Fork" name="par" source="//@scenario.1/@step.9" target="//@scenario.1/@step.6

//@scenario.1/@step.19"/>

<pathConnection xsi:type="Join" name="par" source="//@scenario.1/@step.14 //@scenario.1/@step.16"

target="//@scenario.1/@step.15"/>

<pathConnection xsi:type="Fork" name="log" source="//@scenario.1/@step.19" target="//@scenario.1/@step.4

//@scenario.1/@step.7"/> 
<pathConnection xsi:type="StepSequence" source="//@scenario.1/@step.0" target="//@scenario.1/@step.1"/> <pathConnection xsi:type="StepSequence" source="//@scenario.1/@step.12" target="//@scenario.1/@step.13"/> <pathConnection xsi:type="StepSequence" source="//@scenario.1/@step.10" target="//@scenario.1/@step.14"/> <pathConnection xsi:type="StepSequence" source="//@scenario.1/@step.11" target="//@scenario.1/@step.16"/> <pathConnection xsi:type="StepSequence" source="//@scenario.1/@step.3" target="//@scenario.1/@step.17"/> <pathConnection xsi:type="StepSequence" source="//@scenario.1/@step.17" target="//@scenario.1/@step.18"/> $<$ scenario $>$ $<$ refinement parent="//@scenario.0/@step.5" sub="//@scenario.1"/> $</$ Model $>$

\section{A.9 Video Store}

$<$ ?xml version="1.0" encoding="ISO-8859-1"?> <Model xmi:version="2.0" xmins:xmi="http://www.omg.org/XMI" xmins:xsi="http://www.w3.org/2001/XMLSchemainstance" $\underline{\text { xmlns }=" p l a t f o r m: / r e s o u r c e / M M 2 C S M / M e t a m o d e l s / C S M . e c o r e " ~ n a m e=" V i d e o S t o r e " ~>~}$

<scenario name="VideoStore" > <step name="loop" predecessor="//@scenario.0/@pathConnection.2"

successor="//@scenario.0/@pathConnection.3" refinement="//@refinement.0"/> <step xsi:type="Start" name="start" successor="//@scenario.0/@pathConnection.0" workload="//@workload.0"/> <step xsi:type="ResourceAcquire" name="ra: VideoController" predecessor="//@scenario.0/@pathConnection.0" successor="//@scenario.0/@pathConnection.2" acquire="//@resource.0"/> <step xsi:type="ResourceRelease" name="rr: VideoController" predecessor="//@scenario.0/@pathConnection.3" successor="//@scenario.0/@pathConnection.1" release="//@resource.0"/> <step xsi:type="End" name="end" predecessor="//@scenario.0/@pathConnection.1"/> <pathConnection xsi:type="StepSequence" source="//@scenario.0/@step.1" target="//@scenario.0/@step.2"/> <pathConnection xsi:type="StepSequence" source="//@scenario.0/@step.3" target="//@scenario.0/@step.4"/> <pathConnection xsi:type="StepSequence" source="//@scenario.0/@step.2" target="//@scenario.0/@step.0"/> <pathConnection xsi:type="StepSequence" source="//@scenario.0/@step.0" target="//@scenario.0/@step.3"/> $<$ /scenario $>$ <scenario name="loop" parent="//@scenario.0/@step.0" > <step xsi:type="Start" name="loop start" successor="//@scenario.1/@pathConnection.12" workload="//@workload.I"/>

<step name="store" predecessor="//@scenario.1/@pathConnection.7"

successor="//@scenario.1/@pathConnection.16" > $<$ hostDemand $>(2, \underline{\mathrm{ms}})</$ hostDemand $>$

$</$ step $>$ <step name="freeBuf" predecessor="//@scenario.1/@pathConnection.8" successor="//@scenario.1/@pathConnection.15"> $<$ hostDemand $>(0.2, \underline{\mathrm{ms}})</$ hostDemand $>$ $</$ step $>$ <step name="getBuffer" predecessor="//@scenario.1/@pathConnection.9" successor="//@scenario.1/@pathConnection.13" > $<$ hostDemand $>(1.5, \underline{\mathrm{ms}})</$ hostDemand $>$

$</$ step $>$

<step name="passImage" predecessor="//@scenario.1/@pathConnection.10"

successor="//@scenario.1/@pathConnection.6" > $<$ hostDemand $>(0.9$, ms $)</$ hostDemand $>$

$</$ step $>$

<step name="getlmage" predecessor="//@scenario.1/@pathConnection.11" successor="//@scenario.1/@pathConnection.10" >

$<$ hostDemand $>(1.5, \underline{\mathrm{ms}})</$ hostDemand $>$ 


\section{$</$ step $>$}

<step name="cycleInit" predecessor="//@scenario.1/@pathConnection.12"

successor="//@scenario.1/@pathConnection.14">

$<$ hostDemand $>(1.8, \mathrm{~ms})</$ hostDemand $>$

$</$ step $>$

<step xsi:type="ResourceAcquire" name="ra: StoreProc" predecessor="//@scenario.1/@pathConnection.0"

successor="//@scenario.1/@pathConnection.1" acquire="//@resource.4"/>

<step name="storeImage" predecessor="//@scenario.1/@pathConnection.1"

successor="//@scenario.1/@pathConnection.7">

$<$ hostDemand $>(1.1, \underline{\mathrm{ms}})</$ hostDemand $>$

$</$ step $>$

<step xsi:type="ResourceRelease" name="rr: GetImage" predecessor="//@scenario.1/@pathConnection.6"

successor="//@scenario.1/@pathConnection.0" release="//@resource.3"/>

<step xsi:type="ResourceAcquire" name="ra: BufferManager" predecessor="//@scenario.1/@pathConnection.13"

successor="//@scenario.1/@pathConnection.2" acquire="//@resource.1"/>

<step xsi:type="ResourceAcquire" name="ra: GetImage" predecessor="//@scenario.1/@pathConnection.14"

successor="//@scenario.1/@pathConnection.3" acquire="//@resource.3"/>

<step xsi:type="ResourceAcquire" name="ra: BufferManager" predecessor="//@scenario.1/@pathConnection.15"

successor="//@scenario.1/@pathConnection.4" acquire="//@resource.1"/>

<step xsi:type="ResourceAcquire" name="ra: Database" predecessor="//@scenario.1/@pathConnection.16"

successor="//@scenario.1/@pathConnection.5" acquire="//@resource.2"/>

<step name="allocBuf" predecessor="//@scenario.1/@pathConnection.2"

successor="//@scenario.1/@pathConnection.17" >

$<$ hostDemand $>(0.5, \underline{\mathrm{ms}})</$ hostDemand $>$

$</$ step $>$

<step name="procOneImage" predecessor="//@scenario.1/@pathConnection.3"

successor="//@scenario.1/@pathConnection.9">

$<$ hostDemand $>(1.8, \underline{\mathrm{ms}})</$ hostDemand >

$</$ step $>$

<step name="releaseBuf" predecessor="//@scenario.1/@pathConnection.4"

successor="//@scenario.1/@pathConnection.18">

$<$ hostDemand $>(0.5, \mathrm{~ms})</$ hostDemand $>$

$</$ step $>$

<step name="writeImg" predecessor="//@scenario.1/@pathConnection.5"

successor="//@scenario.1/@pathConnection.19" >

$<$ hostDemand $>(0.9, \mathrm{~ms})</$ hostDemand $>$

$</$ step $>$

<step xsi:type="ResourceRelease" name="rr: BufferManager" predecessor="//@scenario.1/@pathConnection.17" successor="//@scenario.1/@pathConnection.11" release="//@resource.1"/>

<step xsi:type="ResourceRelease" name="rr: BufferManager" predecessor="//@scenario.1/@pathConnection.18"

successor="//@scenario.1/@pathConnection.20" release="//@resource.1"/>

<step xsi:type="ResourceRelease" name="rr: GetImage" predecessor="//@scenario.1/@pathConnection.6"

successor="//@scenario.1/@pathConnection.22" release="//@resource.3"/>

<step xsi:type="ResourceRelease" name="rr: Database" predecessor="//@scenario.1/@pathConnection.19"

successor="//@scenario.1/@pathConnection.8" release="//@resource.2"/>

<step xsi:type="ResourceRelease" name="rr: StoreProc" predecessor="//@scenario.1/@pathConnection.20"

successor="//@scenario.1/@pathConnection.21" release="//@resource.4"/>

<step xsi:type="End" name="end" predecessor="//@scenario.1/@pathConnection.21"/>

<step xsi:type="End" name="loop end" predecessor="//@scenario.1/@pathConnection.6"/>

<step xsi:type="End" name="loop end" predecessor="//@scenario.1/@pathConnection.22"/>

<pathConnection xsi:type="StepSequence" source="//@scenario.1/@step.9" target="//@scenario.1/@step.7"/>

<pathConnection xsi:type="StepSequence" source="//@scenario.1/@step.7"target="//@scenario.1/@step.8"/>

<pathConnection xsi:type="StepSequence" source="//@scenario.1/@step.10" target="//@scenario.1/@step.14"/>

<pathConnection xsi:type="StepSequence" source="//@scenario.1/@step.11" target="//@scenario.1/@step.15"/> 
<pathConnection xsi:type="StepSequence" source="//@scenario.1/@step.12" target="//@scenario.1/@step.16"/> <pathConnection xsi:type="StepSequence" source="//@scenario.1/@step.13" target="//@scenario.1/@step.17"/> <pathConnection xsi:type="Fork" name="storeImage" source="//@scenario.1/@step.4"

target="//@scenario.1/@step.9//@scenario.1/@step.20"/>

<pathConnection xsi:type="StepSequence" source="//@scenario.1/@step.8" target="//@scenario.1/@step.1"/> <pathConnection xsi:type="StepSequence" source="//@scenario.1/@step.21" target="//@scenario.1/@step.2"/> <pathConnection xsi:type="StepSequence" source="//@scenario.1/@step.15" target="//@scenario.1/@step.3"/> <pathConnection xsi:type="StepSequence" source="//@scenario.1/@step.5" target="//@scenario.1/@step.4"/> <pathConnection xsittype="StepSequence" source="//@scenario.1/@step.18" target="//@scenario.1/@step.5"/> <pathConnection xsi:type="StepSequence" source="//@scenario.1/@step.0" target="//@scenario.1/@step.6"/> <pathConnection xsi:type="StepSequence" source="//@scenario.1/@step.3" target="//@scenario.1/@step.10"/> <pathConnection xsi:type="StepSequence" source="//@scenario.1/@step.6" target="//@scenario.1/@step.11"/> <pathConnection xsi:type="StepSequence" source="//@scenario.1/@step.2" target="//@scenario.1/@step.12"/> <pathConnection xsi:type="StepSequence" source="//@scenario.1/@step.1" target="//@scenario.1/@step.13"/> <pathConnection xsi:type="StepSequence" source="//@scenario.1/@step.14" target="//@scenario.1/@step.18"/> <pathConnection xsi:type="StepSequence" source="//@scenario.1/@step.16" target="//@scenario.1/@step.19"/> <pathConnection xsi:type="StepSequence" source="//@scenario.1/@step.17" target="//@scenario.1/@step.21"/> <pathConnection xsi:type="StepSequence" source="//@scenario.1/@step.19" target="//@scenario.1/@step.22"/> <pathConnection xsi:type="StepSequence" source="//@scenario.1/@step.22" target="//@scenario.1/@step.23"/> <pathConnection xsi:type="StepSequence" source="//@scenario.1/@step.20" target="//@scenario.1/@step.25"/> $<$ scenario $>$

$<$ workload $>$

$<$ arrivalPattern $>$ closed </arrivalPattern $>$

$</$ workload $>$

$<$ workload/>

$<$ refinement parent="//@scenario.0/@step.0" sub="//@scenario.1"/>

$<$ resource xsi:type $=$ "Component" name $=$ "VideoController" isActive $=$ "false" $/>$

$<$ resource xsi:type $=$ "Component" name $=$ "BufferManager" isActive $=$ "false" $/>$

$<$ resource $x$ si:type $=$ "Component" name $=$ "Database" isActive $=$ "false" $/>$

$<$ resource xsi:type $=$ "Component" name $=$ "GetImage" isActive $="$ true" $/>$

$<$ resource xsi:type="Component" name="StoreProc" isActive $=$ "true"/>

</Model> 